\title{
Performance of the DELPHI Detector
}

\author{
DELPHI Collaboration
}

\begin{abstract}
DELPHI (DEtector with Lepton, Photon and Hadron Identification) is a detector for $e^{+} e^{-}$physics, designed to provide high granularity over a $4 \pi$ solid angle, allowing an effective particle identification. It has been operating at the LEP (Large Electron-Positron) collider at CERN since 1989. This article reviews its performance.
\end{abstract}


P.Abreu ${ }^{21}$, W.Adam ${ }^{50}$, T.Adye ${ }^{37}$, E.Agasi ${ }^{31}$, I.Ajinenko ${ }^{42}$, R.Aleksan ${ }^{39}$, G.D.Alekseev ${ }^{16}$, R.Alemany ${ }^{49}$, P.P.Allport ${ }^{22}$, S.Almehed ${ }^{24}$, S.J.Alvsvaag ${ }^{4}$, U.Amaldi ${ }^{9}$, S.Amato ${ }^{47}$, A.Andreazza ${ }^{28}$, M.L.Andrieux ${ }^{14}$, P.Antilogus $^{9}$, W-D.Apel ${ }^{17}$, Y.Arnoud ${ }^{39}$, B. ssman $^{44}$, J-E.Augustin ${ }^{19}$, A.Augustinus ${ }^{31}$, P.Baillon ${ }^{9}$, P.Bambade ${ }^{19}$, F.Barao $^{21}$, R.Barate ${ }^{14}$, G.Barbiellini ${ }^{46}$, D.Y.Bardin ${ }^{16}$, A.Baroncelli ${ }^{40}$, O.Barring ${ }^{24}$, J.A.Barrio ${ }^{26}$, W.Bartl ${ }^{50}$, M.J.Bates ${ }^{37}$, M.Battaglia ${ }^{15}$, M.Baubillier ${ }^{23}$, J.Baudot ${ }^{39}$, K-H.Becks ${ }^{52}$, M.Begalli ${ }^{6}$, P.Beilliere ${ }^{8}$, Yu.Belokopytov ${ }^{9,53}$, A.C.Benvenuti ${ }^{5}$, M.Berggren ${ }^{47}$, D.Bertrand ${ }^{2}$, F.Bianchi ${ }^{45}$, M.Bigi $^{45}$, M.S.Bilenky $^{16}$, $^{2}$, P.Billoir $^{23}$, D.Bloch ${ }^{10}$, M.Blume ${ }^{52}$, S.Blyth ${ }^{35}$, T.Bolognese ${ }^{39}$, M.Bonesini ${ }^{28}$, W.Bonivento ${ }^{28}$, P.S.L.Booth ${ }^{22}$, C.Bosio $^{40}$, S.Bosworth ${ }^{35}$, O.Botner ${ }^{48}$, B.Bouquet ${ }^{19}$, C.Bourdarios ${ }^{9}$, T.J.V.Bowcock ${ }^{22}$, M.Bozzo ${ }^{13}$, P.Branchini $^{40}$, K.D.Brand ${ }^{36}$, T.Brenke ${ }^{52}$, R.A.Brenner ${ }^{15}$, C.Bricman ${ }^{2}$, L.Brillault ${ }^{23}$, R.C.A.Brown ${ }^{9}$, P.Bruckman $^{18}$, J-M.Brunet ${ }^{8}$, L.Bugge ${ }^{33}$, T.Buran ${ }^{33}$, T.Burgsmueller ${ }^{52}$, P.Buschmann ${ }^{52}$, A.Buys ${ }^{9}$, S.Cabrera ${ }^{49}$, M.Caccia ${ }^{28}$, M.Calvi $^{28}$, A.J.Camacho Rozas ${ }^{41}$, T.Camporesi ${ }^{9}$, V.Canale ${ }^{38}$, M.Canepa ${ }^{13}$, K.Cankocak $^{44}$, F.Cao $^{2}$, F.Carena ${ }^{9}$, P.Carrilho ${ }^{47}$, L.Carroll ${ }^{22}$, C.Caso $^{13}$, M.V.Castillo Gimenez ${ }^{49}$, A.Cattai ${ }^{9}$, F.R.Cavallo ${ }^{5}$, L.Cerrito $^{38}{ }^{3}$, V.Chabaud $^{9}$, Ph.Charpentier ${ }^{9}$, L.Chaussard ${ }^{25}$, J.Chauveau ${ }^{23}$, P.Checchia ${ }^{36}$, G.A.Chelkov ${ }^{16}$, M.Chen ${ }^{2}$, R.Chierici $^{45}$, P.Chliapnikov ${ }^{42}$, P.Chochula ${ }^{7}$, V.Chorowicz ${ }^{9}$, J.Chudoba ${ }^{30}$, V.Cindro ${ }^{43}$, P.Collins ${ }^{9}$, J.L.Contreras ${ }^{19}$, R.Contri ${ }^{13}$, E.Cortina ${ }^{49}$, G.Cosme ${ }^{19}$, F.Cossutti ${ }^{46}$, H.B.Crawley ${ }^{1}$, D.Crennell ${ }^{37}$, G.Crosetti $^{13}$, J.Cuevas Maestro $^{34}$, S.Czellar ${ }^{15}$, E.Dahl-Jensen ${ }^{29}$, J.Dahm ${ }^{52}$, B.Dalmagne ${ }^{19}$, M.Dam ${ }^{29}$, G.Damgaard ${ }^{29}$, P.D.Dauncey ${ }^{37}$, M.Davenport ${ }^{9}$, W.Da Silva ${ }^{23}$, C.Defoix ${ }^{8}$, A.Deghorain ${ }^{2}$, G.Della Ricca ${ }^{46}$, P.Delpierre ${ }^{27}$, N.Demaria ${ }^{35}$, A.De Angelis ${ }^{9}$, W.De Boer ${ }^{17}$, S.De Brabandere ${ }^{2}$, C.De Clercq ${ }^{2}$, C.De La Vaissiere ${ }^{23}$, B.De Lotto ${ }^{46}$, A.De Min ${ }^{36}$, L.De Paula ${ }^{47}$, C.De Saint-Jean ${ }^{39}$, H.Dijkstra ${ }^{9}$, L.Di Ciaccio ${ }^{38}$, F.Djama $^{10}$, J.Dolbeau $^{8}$, M.Donszelmann ${ }^{9}$, K.Doroba ${ }^{51}$, M.Dracos ${ }^{10}$, J.Drees ${ }^{52}$, K.-A.Drees ${ }^{52}$, M.Dris ${ }^{32}$, Y.Dufour ${ }^{9}$, F.Dupont ${ }^{14}$, D.Edsall ${ }^{1}$, R.Ehret ${ }^{17}$, G.Eigen ${ }^{4}$, T.Ekelof ${ }^{48}$, G.Ekspong ${ }^{44}$, M.Elsing ${ }^{52}$, J-P.Engel ${ }^{10}$, N.Ershaidat ${ }^{23}$, B.Erzen ${ }^{43}$, M.Espirito Santo ${ }^{21}$, V.Falaleev ${ }^{42}$, E.Falk ${ }^{24}$, D.Fassouliotis ${ }^{32}$, M.Feindt ${ }^{9}$, A.Fenyuk ${ }^{42}$, A.Ferrer ${ }^{49}$, T.A.Filippas ${ }^{32}$, A.Firestone ${ }^{1}$, P.-A.Fischer ${ }^{10}$, H.Foeth ${ }^{9}$, E.Fokitis ${ }^{32}$, F.Fontanelli ${ }^{13}$, F.Formenti $^{9}$, B.Franek ${ }^{37}$, P.Frenkiel $^{8}$, D.C.Fries ${ }^{17}$, A.G.Frodesen ${ }^{4}$, R.Fruhwirth ${ }^{50}$, F.Fulda-Quenzer ${ }^{19}$, J.Fuster ${ }^{49}$, A.Galloni $^{22}$,

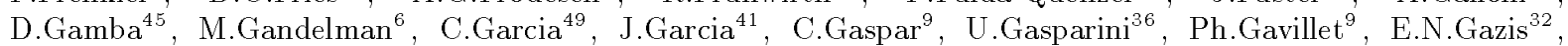
D.Gele ${ }^{10}$, J-P.Gerber ${ }^{10}$, L.Gerdyukov ${ }^{42}$, M.Gibbs ${ }^{22}$, R.Gokieli ${ }^{51}$, B.Golob ${ }^{43}$, G.Gopal ${ }^{37}$, L.Gorn ${ }^{1}$, M.Gorski ${ }^{51}$, Yu.Gouz ${ }^{45,53}$, V.Gracco ${ }^{13}$, E.Graziani ${ }^{40}$, G.Grosdidier ${ }^{19}$, K.Grzelak ${ }^{51}$, S.Gumenyuk ${ }^{28,53}$, P.Gunnarsson ${ }^{44}$, M.Gunther $^{48}$, J.Guy ${ }^{37}$, F.Hahn ${ }^{9}$, S.Hahn ${ }^{52}$, Z.Hajduk ${ }^{18}$, A.Hallgren ${ }^{48}$, K.Hamacher ${ }^{52}$, W.Hao ${ }^{31}$, F.J.Harris ${ }^{35}$, V.Hedberg $^{24}$, R.Henriques ${ }^{21}$, J.J.Hernandez ${ }^{49}$, P.Herquet ${ }^{2}$, H.Herr ${ }^{9}$, T.L.Hessing ${ }^{35}$, E.Higon ${ }^{49}$, H.J.Hilke $^{9}$, T.S.Hill $^{1}$, S-O.Holmgren ${ }^{44}$, P.J.Holt ${ }^{35}$, D.Holthuizen ${ }^{31}$, S.Hoorelbeke ${ }^{2}$, M.Houlden ${ }^{22}$, J.Hrubec ${ }^{50}$, K.Huet ${ }^{2}$, K.Hultqvist ${ }^{44}$, J.N.Jackson ${ }^{22}$, R.Jacobsson ${ }^{44}$, P.Jalocha ${ }^{18}$, R.Janik ${ }^{7}$, Ch.Jarlskog ${ }^{24}$, G.Jarlskog ${ }^{24}$, P.Jarry ${ }^{39}$, B.Jean-Marie ${ }^{19}$, E.K.Johansson ${ }^{44}$, L.Jonsson ${ }^{24}$, P.Jonsson ${ }^{24}$, C.Joram ${ }^{9}$, P.Juillot ${ }^{10}$, M.Kaiser $^{17}$, F.Kapusta ${ }^{23}$, K.Karafasoulis $^{11}$, M.Karlsson ${ }^{44}$, E.Karvelas ${ }^{11}$, A.Karyukhin ${ }^{42}$, S.Katsanevas ${ }^{3}$, E.C.Katsoufis ${ }^{32}$, R.Keranen $^{44}$, B.A.Khomenko $^{16}$, N.N.Khovanski ${ }^{16}$, B.King ${ }^{22}$, N.J.Kjaer ${ }^{29}$, H.Klein ${ }^{9}$, A.Klovning ${ }^{4}$, P.Kluit ${ }^{31}$, B.Koene ${ }^{31}$, P.Kokkinias $^{11}$, M.Koratzinos ${ }^{9}$, C.Kourkoumelis ${ }^{3}$, O.Kouznetsov ${ }^{13,16}$, P.-H.Kramer ${ }^{52}$, M.Krammer ${ }^{50}$, C.Kreuter $^{17}$, I.Kronkvist ${ }^{24}$, Z.Krumstein ${ }^{16}$, W.Krupinski ${ }^{18}$, P.Kubinec ${ }^{7}$, W.Kucewicz ${ }^{18}$, K.Kurvinen $^{15}$, C.Lacasta $^{49}$, I.Laktineh ${ }^{25}$, S.Lamblot $^{23}$, J.W.Lamsa ${ }^{1}$, L.Lanceri ${ }^{46}$, D.W.Lane ${ }^{1}$, P.Langefeld ${ }^{52}$, V.Lapin $^{42}$, I.Last $^{22}$, J-P.Laugier ${ }^{39}$, R.Lauhakangas ${ }^{15}$, G.Leder ${ }^{50}$, F.Ledroit ${ }^{14}$, V.Lefebure ${ }^{2}$, C.K.Legan ${ }^{1}$, R.Leitner ${ }^{30}$, Y.Lemoigne $^{39}$, J.Lemonne ${ }^{2}$, G.Lenzen ${ }^{52}$, V.Lepeltier ${ }^{19}$, T.Lesiak ${ }^{36}$, D.Liko ${ }^{50}$, R.Lindner ${ }^{52}$, A.Lipniacka ${ }^{36}$, I.Lippi $^{36}$, B.Loerstad ${ }^{24}$, J.G.Loken ${ }^{35}$, J.M.Lopez ${ }^{41}$, M.A.Lopez Aguera ${ }^{41}$, D.Loukas ${ }^{11}$, P.Lutz ${ }^{39}$, L.Lyons ${ }^{35}$, J.MacNaughton $^{50}$, G.Maehlum ${ }^{17}$, A.Maio ${ }^{21}$, V.Malychev ${ }^{16}$, F.Mandl ${ }^{50}$, J.Marco ${ }^{41}$, B.Marechal ${ }^{47}$, M.Margoni $^{36}$, J-C.Marin $^{9}$, C.Mariott ${ }^{40}$, A.Markou ${ }^{11}$, T.Maron ${ }^{52}$, C.Martinez-Rivero ${ }^{41}$, F.Martinez-Vidal ${ }^{49}$, S.Marti i Garcia ${ }^{49}$, J.Masik ${ }^{30}$, F.Matorras ${ }^{41}$, C.Matteuzzi ${ }^{9}$, G.Matthiae ${ }^{38}$, M.Mazzucato ${ }^{36}$, M.Mc Cubbin ${ }^{9}$, R.Mc Kay $^{1}$, R.Mc Nulty ${ }^{22}$, J.Medbo ${ }^{48}$, C.Meroni ${ }^{28}$, S.Meyer ${ }^{17}$, W.T.Meyer ${ }^{1}$, M.Michelotto ${ }^{36}$, E.Migliore ${ }^{45}$, L.Mirabito $^{25}$, W.A.Mitaroff ${ }^{50}$, U.Mjoernmark ${ }^{24}$, T.Moa ${ }^{44}$, R.Moeller ${ }^{29}$, K.Moenig ${ }^{9}$, M.R.Monge ${ }^{13}$, P.Morettini $^{13}$, H.Mueller ${ }^{17}$, L.M.Mundim ${ }^{6}$, W.J.Murray ${ }^{37}$, B.Muryn ${ }^{18}$, G.Myatt ${ }^{35}$, F.Naraghi ${ }^{14}$, F.L.Navarria ${ }^{5}$, S.Navas $^{49}$, K.Nawrocki ${ }^{51}$, P.Negri ${ }^{28}$, W.Neumann ${ }^{52}$, N.Neumeister ${ }^{50}$, R.Nicolaidou ${ }^{3}$, B.S.Nielsen ${ }^{29}$, M.Nieuwenhuizen $^{31}$, V.Nikolaenko ${ }^{10}$, P.Niss ${ }^{44}$, A.Nomerotski ${ }^{36}$, A.Normand ${ }^{35}$, W.Oberschulte-Beckmann ${ }^{17}$, V.Obraztsov $^{42}$, A.G.Olshevski ${ }^{16}$, A.Onofre ${ }^{21}$, R.Orava ${ }^{15}$, K.Osterberg ${ }^{15}$, A.Ouraou ${ }^{39}$, P.Paganini ${ }^{19}$, M.Paganoni ${ }^{9}$, P.Pages $^{10}$, H.Palka ${ }^{18}$, Th.D.Papadopoulou ${ }^{32}$, K.Papageorgiou ${ }^{11}$, L.Pape ${ }^{9}$, C.Parkes ${ }^{35}$, F.Parodi ${ }^{13}$, A.Passeri ${ }^{40}$, M.Pegoraro ${ }^{36}$, L.Peralta ${ }^{21}$, V.Perevozchikov ${ }^{42}$, H.Pernegger ${ }^{50}$, A.Perrotta ${ }^{5}$, C.Petridou $^{46}$, A.Petrolini ${ }^{13}$, M.Petrovyck ${ }^{28,53}$, H.T.Phillips ${ }^{37}$, G.Piana ${ }^{13}$, F.Pierre ${ }^{39}$, M.Pimenta ${ }^{21}$, M.Pindo ${ }^{28}$, S.Plaszczynski $^{19}$, O.Podobrin ${ }^{17}$, M.E.Pol ${ }^{6}$, G.Polok ${ }^{18}$, P.Poropat ${ }^{46}$, V.Pozdniakov ${ }^{16}$, M.Prest ${ }^{46}$, P.Privitera ${ }^{38}$, N.Pukhaeva ${ }^{16}$, A.Pullia ${ }^{28}$, D.Radojicic ${ }^{35}$, S.Ragazzi ${ }^{28}$, H.Rahmani ${ }^{32}$, J.Rames ${ }^{12}$, P.N.Ratoff ${ }^{20}$, A.L.Read ${ }^{33}$, M.Reale $^{52}$, P.Rebecchi ${ }^{19}$, N.G.Redaelli ${ }^{28}$, M.Regler ${ }^{50}$, D.Reid ${ }^{9}$, P.B.Renton ${ }^{35}$, L.K.Resvanis ${ }^{3}$, F.Richard ${ }^{19}$, J.Richardson $^{22}$, J.Ridky ${ }^{12}$, G.Rinaudo ${ }^{45}$, I.Ripp ${ }^{39}$, A.Romero ${ }^{45}$, I.Roncagliolo ${ }^{13}$, P.Ronchese ${ }^{36}$, L.Roos ${ }^{14}$, E.I.Rosenberg ${ }^{1}$, E.Rosso ${ }^{9}$, P.Roudeau ${ }^{19}$, T.Rovelli ${ }^{5}$, W.Ruckstuhl ${ }^{31}$, V.Ruhlmann-Kleider ${ }^{39}$, A.Ruiz ${ }^{41}$, K.Rybicki $^{18}$, H.Saarikko ${ }^{15}$, Y.Sacquin ${ }^{39}$, A.Sadovsky ${ }^{16}$, G.Sajot ${ }^{14}$, J.Salt ${ }^{49}$, J.Sanchez ${ }^{26}$, M.Sannino ${ }^{13}$, M.Schimmelpfennig ${ }^{17}$, H.Schneider ${ }^{17}$, U.Schwickerath ${ }^{17}$, M.A.E.Schyns ${ }^{52}$, G.Sciolla ${ }^{45}$, F.Scuri ${ }^{46}$, P.Seager $^{20}$, Y.Sedykh $^{16}$, A.M.Segar ${ }^{35}$, A.Seitz ${ }^{17}$, R.Sekulin ${ }^{37}$, R.C.Shellard ${ }^{6}$, I.Siccama ${ }^{31}$, P.Siegrist ${ }^{39}$, S.Simonetti $^{39}$, F.Simonetto $^{36}$, A.N.Sisakian ${ }^{16}$, B.Sitar ${ }^{7}$, T.B.Skaali ${ }^{33}$, G.Smadja ${ }^{25}$, N.Smirnov ${ }^{42}$, O.Smirnova ${ }^{16}$, G.R.Smith ${ }^{37}$, O.Solovianov ${ }^{42}$, R.Sosnowski ${ }^{51}$, D.Souza-Santos ${ }^{6}$, E.Spiriti ${ }^{40}$, P.Sponholz ${ }^{52}$, S.Squarcia ${ }^{13}$, C.Stanescu ${ }^{40}$, S.Stapnes $^{33}$, I.Stavitski ${ }^{36}$, F.Stichelbaut ${ }^{9}$, A.Stocchi ${ }^{19}$, J.Strauss ${ }^{50}$, R.Strub ${ }^{10}$, B.Stugu ${ }^{4}$, M.Szczekowski ${ }^{51}$, 
M.Szeptycka $^{51}$, T.Tabarelli ${ }^{28}$, J.P.Tavernet ${ }^{23}$, O.Tchikilev ${ }^{42}$, A.Tilquin ${ }^{27}$, J.Timmermans ${ }^{31}$, L.G.Tkatchev ${ }^{16}$, T.Todorov $^{10}$, D.Z.Toet ${ }^{31}$, A.Tomaradze ${ }^{2}$, B.Tome ${ }^{21}$, A.Tonazzo ${ }^{28}$, L.Tortora ${ }^{40}$, G.Transtromer ${ }^{24}$, D.Treille ${ }^{9}$, W.Trischuk ${ }^{9}$, G.Tristram ${ }^{8}$, A.Trombini ${ }^{19}$, C.Troncon ${ }^{28}$, A.Tsirou ${ }^{9}$, M-L.Turluer ${ }^{39}$, I.A.Tyapkin ${ }^{16}$, M.Tynde $^{37}$, S.Tzamarias $^{22}$, B.Ueberschaer ${ }^{52}$ O.Ullaland ${ }^{9}$, G.Valenti ${ }^{5}$ E.Vallazza ${ }^{9}$ C.Vander Velde ${ }^{2}$, G.W.Van Apeldoorn ${ }^{31}$, P.Van Dam ${ }^{31}$, W.K.Van Doninck ${ }^{2}$, J.Van Eldik ${ }^{31}$, N.Vassilopoulos ${ }^{35}$, G.Vegni ${ }^{28}$, L.Ventura $^{36}$, W.Venus ${ }^{37}$, F.Verbeure ${ }^{2}$, M.Verlato ${ }^{36}$, L.S.Vertogradov ${ }^{16}$, D.Vilanova ${ }^{39}$, P.Vincent ${ }^{25}$, L.Vitale ${ }^{46}$, E.Vlasov ${ }^{42}$, A.S.Vodopyanov ${ }^{16}$, V.Vrba ${ }^{12}$, H.Wahlen ${ }^{52}$, C.Walck ${ }^{44}$, M.Weierstall ${ }^{52}$, P.Weilhammer ${ }^{9}$, C.Weiser $^{17}$, A.M.Wetherell $^{9}$, D.Wicke ${ }^{52}$, J.H.Wickens ${ }^{2}$, M.Wielers ${ }^{17}$, G.R.Wilkinson ${ }^{35}$, W.S.C.Williams ${ }^{35}$, M.Winter ${ }^{10}$, M.Witek $^{18}$, K.Woschnagg ${ }^{48}$, K.Yip ${ }^{35}$, F.Zach ${ }^{25}$, A.Zaitsev ${ }^{42}$, A.Zalewska ${ }^{18}$, P.Zalewski ${ }^{51}$, D.Zavrtanik ${ }^{43}$, E.Zevgolatakos ${ }^{11}$, N.I.Zimin ${ }^{16}$, M.Zito ${ }^{39}$, D.Zontar ${ }^{43}$, R.Zuberi ${ }^{35}$, G.C.Zucchelli ${ }^{44}$, G.Zumerle ${ }^{36}$

\footnotetext{
${ }^{1}$ Ames Laboratory and Department of Physics, Iowa State University, Ames IA 50011, USA

${ }^{2}$ Physics Department, Univ. Instelling Antwerpen, Universiteitsplein 1, B-2610 Wilrijk, Belgium and IIHE, ULB-VUB, Pleinlaan 2, B-1050 Brussels, Belgium

and Faculté des Sciences, Univ. de l'Etat Mons, Av. Maistriau 19, B-7000 Mons, Belgium

${ }^{3}$ Physics Laboratory, University of Athens, Solonos Str. 104, GR-10680 Athens, Greece

${ }^{4}$ Department of Physics, University of Bergen, Allégaten 55, N-5007 Bergen, Norway

${ }^{5}$ Dipartimento di Fisica, Università di Bologna and INFN, Via Irnerio 46, I-40126 Bologna, Italy

${ }^{6}$ Centro Brasileiro de Pesquisas Físicas, rua Xavier Sigaud 150, RJ-22290 Rio de Janeiro, Brazil and Depto. de Física, Pont. Univ. Católica, C.P. 38071 RJ-22453 Rio de Janeiro, Brazil and Inst. de Física, Univ. Estadual do Rio de Janeiro, rua São Francisco Xavier 524, Rio de Janeiro, Brazil

${ }^{7}$ Comenius University, Faculty of Mathematics and Physics, Mlynska Dolina, SK-84215 Bratislava, Slovakia

${ }^{8}$ Collège de France, Lab. de Physique Corpusculaire, IN2P3-CNRS, F-75231 Paris Cedex 05, France

${ }^{9} \mathrm{CERN}, \mathrm{CH}-1211$ Geneva 23, Switzerland

${ }^{10}$ Centre de Recherche Nucléaire, IN2P3 - CNRS/ULP - BP20, F-67037 Strasbourg Cedex, France

${ }^{11}$ Institute of Nuclear Physics, N.C.S.R. Demokritos, P.O. Box 60228, GR-15310 Athens, Greece

${ }^{12}$ FZU, Inst. of Physics of the C.A.S. High Energy Physics Division, Na Slovance 2, 180 40, Praha 8, Czech Republic

${ }^{13}$ Dipartimento di Fisica, Università di Genova and INFN, Via Dodecaneso 33, I-16146 Genova, Italy

${ }^{14}$ Institut des Sciences Nucléaires, IN2P3-CNRS, Université de Grenoble 1, F-38026 Grenoble Cedex, France

${ }^{15}$ Research Institute for High Energy Physics, SEFT, P.O. Box 9, FIN-00014 Helsinki, Finland

${ }^{16}$ Joint Institute for Nuclear Research, Dubna, Head Post Office, P.O. Box 79, 101000 Moscow, Russian Federation

${ }^{17}$ Institut für Experimentelle Kernphysik, Universität Karlsruhe, Postfach 6980, D-76128 Karlsruhe, Germany

${ }^{18}$ Institute of Nuclear Physics and University of Mining and Metalurgy, Ul. Kawiory 26a, PL-30055 Krakow, Poland

${ }^{19}$ Université de Paris-Sud, Lab. de l'Accélérateur Linéaire, IN2P3-CNRS, Bât. 200, F-91405 Orsay Cedex, France

${ }^{20}$ School of Physics and Materials, University of Lancaster, Lancaster LA1 4YB, UK

${ }^{21}$ LIP, IST, FCUL - Av. Elias Garcia, 14-1 ${ }^{\circ}$, P-1000 Lisboa Codex, Portugal

${ }^{22}$ Department of Physics, University of Liverpool, P.O. Box 147, Liverpool L69 3BX, UK

${ }^{23}$ LPNHE, IN2P3-CNRS, Universités Paris VI et VII, Tour 33 (RdC), 4 place Jussieu, F-75252 Paris Cedex 05, France

${ }^{24}$ Department of Physics, University of Lund, Sölvegatan 14, S-22363 Lund, Sweden

${ }^{25}$ Université Claude Bernard de Lyon, IPNL, IN2P3-CNRS, F-69622 Villeurbanne Cedex, France

${ }^{26}$ Universidad Complutense, Avda. Complutense s/n, E-28040 Madrid, Spain

${ }^{27}$ Univ. d'Aix - Marseille II - CPP, IN2P3-CNRS, F-13288 Marseille Cedex 09, France

${ }^{28}$ Dipartimento di Fisica, Università di Milano and INFN, Via Celoria 16, I-20133 Milan, Italy

${ }^{29}$ Niels Bohr Institute, Blegdamsvej 17, DK-2100 Copenhagen 0, Denmark

${ }^{30} \mathrm{NC}$, Nuclear Centre of MFF, Charles University, Areal MFF, V Holesovickach 2, 180 00, Praha 8, Czech Republic

${ }^{31}$ NIKHEF-H, Postbus 41882, NL-1009 DB Amsterdam, The Netherlands

${ }^{32}$ National Technical University, Physics Department, Zografou Campus, GR-15773 Athens, Greece

${ }^{33}$ Physics Department, University of Oslo, Blindern, N-1000 Oslo 3, Norway

${ }^{34}$ Dpto. Fisica, Univ. Oviedo, C/P. Pérez Casas, S/N-33006 Oviedo, Spain

${ }^{35}$ Department of Physics, University of Oxford, Keble Road, Oxford OX1 3RH, UK

${ }^{36}$ Dipartimento di Fisica, Università di Padova and INFN, Via Marzolo 8, I-35131 Padua, Italy

${ }^{37}$ Rutherford Appleton Laboratory, Chilton, Didcot OX11 OQX, UK

${ }^{38}$ Dipartimento di Fisica, Università di Roma II and INFN, Tor Vergata, I-00173 Rome, Italy

${ }^{39}$ Centre d'Etudes de Saclay, DSM/DAPNIA, F-91191 Gif-sur-Yvette Cedex, France

${ }^{40}$ Istituto Superiore di Sanità, Ist. Naz. di Fisica Nucl. (INFN), Viale Regina Elena 299, I-00161 Rome, Italy

${ }^{41}$ Instituto de Fisica de Cantabria (CSIC-UC), Avda. los Castros, S/N-39006 Santander, Spain, (CICYT-AEN93-0832)

${ }^{42}$ Inst. for High Energy Physics, Serpukov P.O. Box 35, Protvino, (Moscow Region), Russian Federation

${ }^{43}$ J. Stefan Institute and Department of Physics, University of Ljubljana, Jamova 39, SI-61000 Ljubljana, Slovenia

${ }^{44}$ Fysikum, Stockholm University, Box 6730, S-113 85 Stockholm, Sweden

${ }^{45}$ Dipartimento di Fisica Sperimentale, Università di Torino and INFN, Via P. Giuria 1, I-10125 Turin, Italy

${ }^{46}$ Dipartimento di Fisica, Università di Trieste and INFN, Via A. Valerio 2, I-34127 Trieste, Italy and Istituto di Fisica, Università di Udine, I-33100 Udine, Italy

${ }^{47}$ Univ. Federal do Rio de Janeiro, C.P. 68528 Cidade Univ., Ilha do Fundão BR-21945-970 Rio de Janeiro, Brazil

${ }^{48}$ Department of Radiation Sciences, University of Uppsala, P.O. Box 535, S-751 21 Uppsala, Sweden

${ }^{49}$ IFIC, Valencia-CSIC, and D.F.A.M.N., U. de Valencia, Avda. Dr. Moliner 50, E-46100 Burjassot (Valencia), Spain

${ }^{50}$ Institut für Hochenergiephysik, Österr. Akad. d. Wissensch., Nikolsdorfergasse 18, A-1050 Vienna, Austria

${ }^{51}$ Inst. Nuclear Studies and University of Warsaw, Ul. Hoza 69, PL-00681 Warsaw, Poland

${ }^{52}$ Fachbereich Physik, University of Wuppertal, Postfach 100 127, D-42097 Wuppertal 1, Germany

${ }^{53}$ On leave of absence from IHEP Serpukhov
} 


\section{Contents}

1 Introduction $\quad 1$

2 Trigger $\quad 3$

3 Data Acquisition, Control and Monitoring 4

3.1 Data Acquisition System . . . . . . . . . . . . . . . . . . . . . . . 4

3.2 DAS Control System . . . . . . . . . . . . . . . . . . . . 6

3.3 Slow Controls System . . . . . . . . . . . . . . . . . . . . . . 8

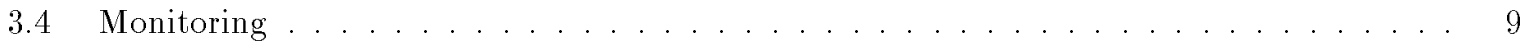

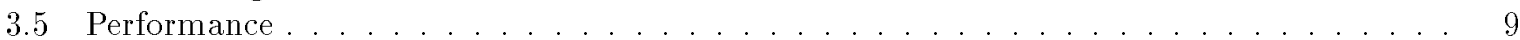

4 Luminosity Measurement $\quad 9$

4.1 The Luminosity Analysis . . . . . . . . . . . . . . . . . . . . . . . . . . . 10

4.1.1 Definition of the Acceptance . . . . . . . . . . . . . . . . . 10

4.1.2 Background Rejection . . . . . . . . . . . . . . . . . . . . . 11

4.2 Results. . . . . . . . . . . . . . . . . . . . . . 11

5 Detector Simulation $\quad 12$

6 Reconstruction Program $\quad 14$

7 Tracking Performance $\quad 15$

7.1 Vertex Detector . . . . . . . . . . . . . . . . . . . . . . 16

7.2 Inner Detector . . . . . . . . . . . . . . . . . . . . . . . . . 17

7.3 Time Projection Chamber . . . . . . . . . . . . . . . . . . . . 18

7.4 Outer Detector . . . . . . . . . . . . . . . . . . . . . . . . . . . 19

7.5 Forward Chamber A . . . . . . . . . . . . . . . . . . . . . 19

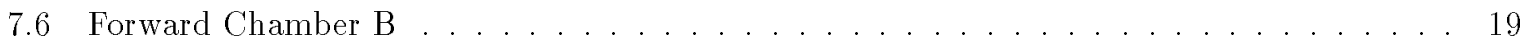

7.7 Global Alignment . . . . . . . . . . . . . . . . . . . . . . . 20

7.8 Momentum Precision . . . . . . . . . . . . . . . . . . . . . . 20

7.9 Charmed Particle Mass Reconstruction _. . . . . . . . . . . . . . . 23

$8 V^{0}$ Reconstruction $\quad 23$

$9 \quad \boldsymbol{b}$ Tagging $\quad \mathbf{2 5}$

9.1 Primary Vertex Reconstruction . . . . . . . . . . . . . . . . . . . 25

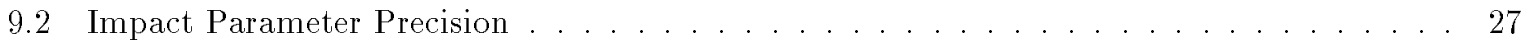

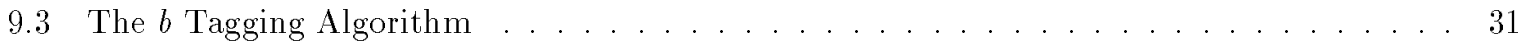

10 Charged Hadron Identification 33

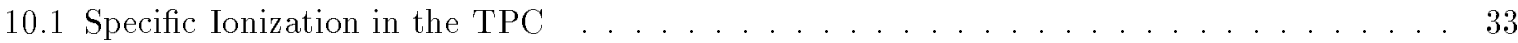

10.2 Ring Imaging Cherenkov Detectors . . . . . . . . . . . . . . . . . . . . . 35

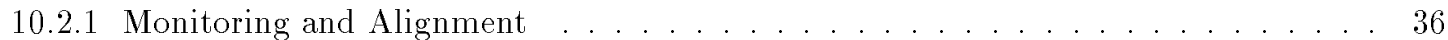

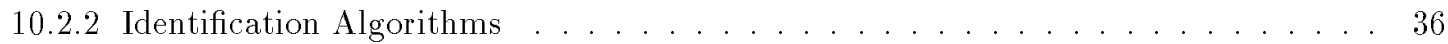

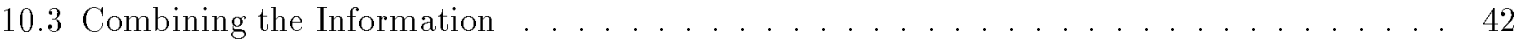

11 Electromagnetic Calorimeters 44

11.1 Barrel Electromagnetic Calorimeter . . . . . . . . . . . . . . . . . . . . . . . 44

11.2 Forward Electromagnetic Calorimeter . . . . . . . . . . . . . . . . . . . 45

11.3 Supplementary Photon Taggers . . . . . . . . . . . . . . . . . . . 47

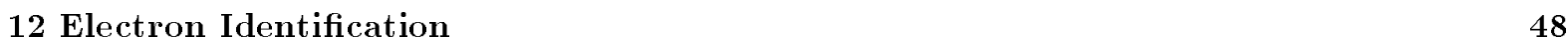

13 Photon and $\boldsymbol{\pi}^{\mathbf{0}}$ Identification $\quad 51$

$13.1 \pi^{0}$ Reconstruction from Single Photons . . . . . . . . . . . . . . . . . 51

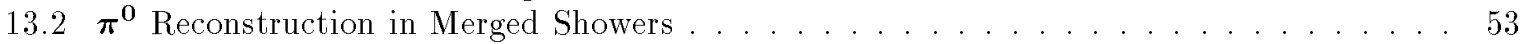

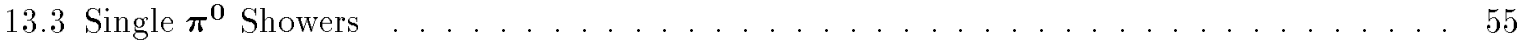


16 Muon Identification 61

17 Event Selection for Physics Analysis 64

17.1 Hadronic Event Selection . . . . . . . . . . . . . . . . . . . . . 64

17.2 Leptonic Event Selection . . . . . . . . . . . . . . . . . . . . . . . 64

$17.2 .1 e^{+} e^{-}$Event Selection . . . . . . . . . . . . . . . . . . . 65

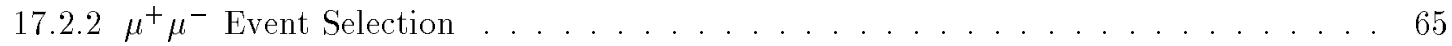

$17.2 .3 \tau^{+} \tau^{-}$Event Selection . . . . . . . . . . . . . . . . . 66 


\section{Introduction}

DELPHI (DEtector with Lepton, Photon and Hadron Identification) is a detector for $e^{+} e^{-}$physics, designed to provide high granularity over a $4 \pi$ solid angle, and allowing powerful particle identification. It is installed at the LEP (Large Electron-Positron) collider at CERN where it has operated since 1989. The numbers of hadronic Z decays recorded each year at LEP1 (the first phase of LEP, with centre of mass energy $E_{c m}<100$ $\mathrm{GeV}$ ) are summarised in Table 1 . The components (subdetectors) present in DELPHI in 1990 were described in detail in [1]. The aim of this article is to summarise developments since then and to review the performance achieved.

\begin{tabular}{|l|c|c|c|c|c|c|c|c|}
\hline Year & 1989 & 1990 & 1991 & 1992 & 1993 & 1994 & 1995 & Total \\
\hline Off peak & $4 \mathrm{~K}$ & $30 \mathrm{~K}$ & $52 \mathrm{~K}$ & - & $243 \mathrm{~K}$ & - & $236 \mathrm{~K}$ & $617 \mathrm{~K}$ \\
Total & $13 \mathrm{~K}$ & $125 \mathrm{~K}$ & $275 \mathrm{~K}$ & $751 \mathrm{~K}$ & $755 \mathrm{~K}$ & $1484 \mathrm{~K}$ & $750 \mathrm{~K}$ & $4153 \mathrm{~K}$ \\
\hline
\end{tabular}

Table 1: Numbers of hadronic Z decays recorded by DELPHI in each year of operation of LEP1, in a running period normally lasting from May to November. The numbers recorded at energies off the peak of the $\mathrm{Z}$ resonance, during scans of the $\mathrm{Z}$ line shape, are noted separately.

In the standard DELPHI coordinate system, the $z$ axis is along the electron direction, the $x$ axis points towards the centre of LEP, and the $y$ axis points upwards. The polar angle to the $z$ axis is called $\theta$ and the azimuthal angle around the $z$ axis is called $\phi$; the radial coordinate is $R=\sqrt{x^{2}+y^{2}}$.

The detector consists of a cylindrical section covering the "barrel" region of $\theta$ (typically from $40^{\circ}$ to $140^{\circ}$ ) and two endcaps covering the "forward" regions. The endcaps can be moved to allow access to the subdetectors. Figure 1 schematically shows the present layout of the barrel and of one endcap.

The superconducting solenoid provides a highly uniform magnetic field of $1.23 \mathrm{~T}$ parallel to the $z$ axis throughout the central tracking volume, i.e. the volume containing the barrel tracking detectors - namely the Vertex Detector (VD), the Inner Detector (ID), the Time Projection Chamber (TPC) and the Outer Detector (OD) - and also the forward tracking chambers (Forward Chambers A and B).

The VD was first installed as a 2-layer silicon-strip detector measuring $R \phi$ in the barrel region. In April 1991 the $8 \mathrm{~cm}$ radius Aluminium beam pipe was replaced by a $5.6 \mathrm{~cm}$ radius Beryllium one and the VD was upgraded [2] by adding a third ("Closer") layer of silicon strips. In April 1994 the VD was further upgraded [3] by adding z readout to the external ("Outer") and Closer layers. At the same time the polar angle coverage of the Closer layer was extended into the forward region, down to 25․ In April 1995 the ID was replaced by a longer one covering polar angles down to $15^{\circ}$. These last two steps were the start of an ongoing upgrade of the tracking in the forward region [4] that will be completed for data-taking in 1996.

Electron and photon identification is provided primarily by the High density Projection Chamber (HPC) in the barrel region and by the Forward Electromagnetic Calorimeter (FEMC) in the endcaps. The smaller polar angles, essential for detecting $e^{+}$and $e^{-}$from $\gamma \gamma$ processes and for luminosity measurement from $e^{+} e^{-} \rightarrow e^{+} e^{-}$events, are covered by the Small angle TIle Calorimeter (STIC) [5], which replaced the Small Angle Tagger (SAT) in April 1994, and the Very Small Angle Tagger (VSAT). In order to achieve complete hermeticity for high energy photon detection, important at LEP2, additional scintillators have now been installed in the cable duct regions, between the barrel and 


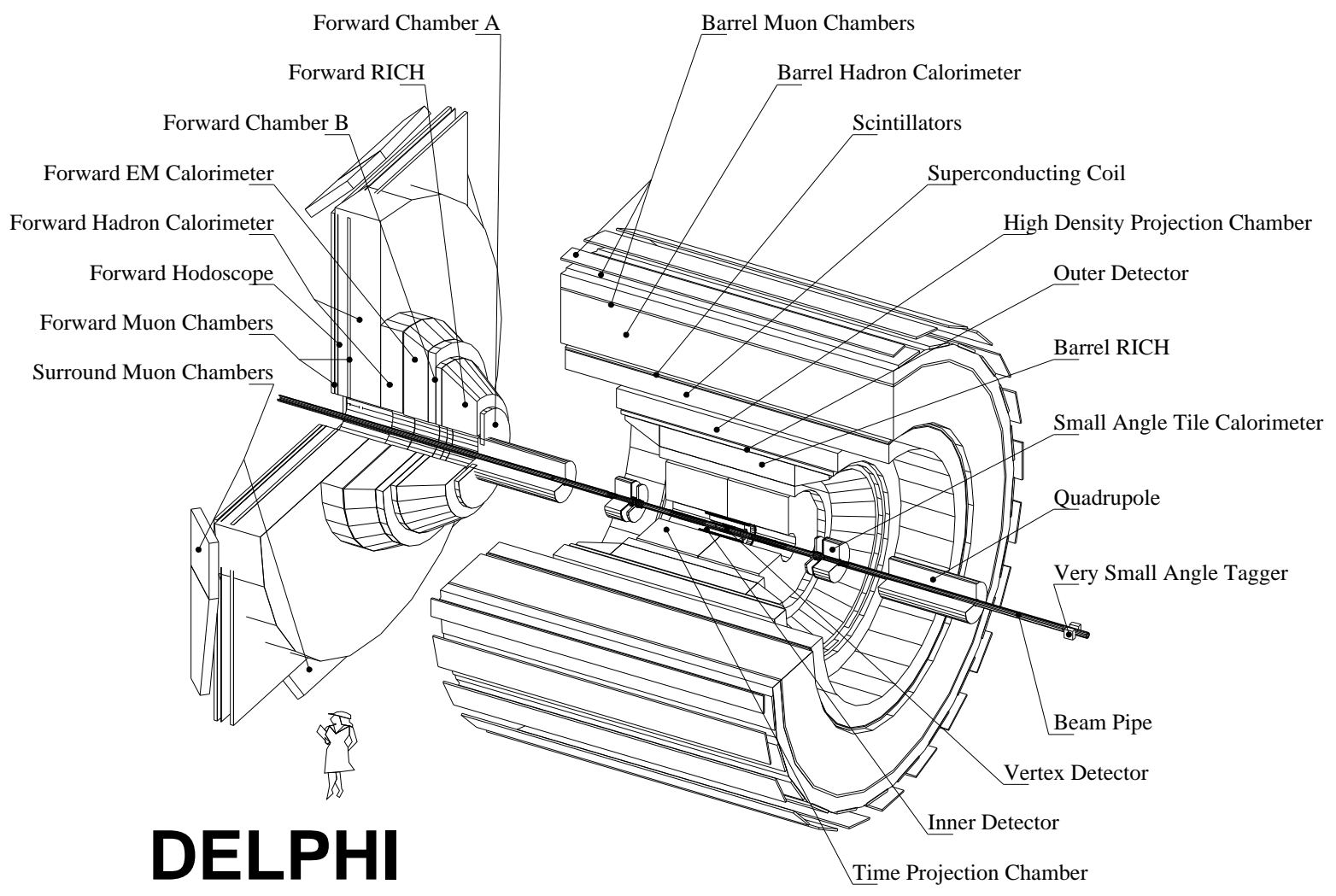

Figure 1: Schematic layout of DELPHI (note that Forward Chamber A is actually fixed to the Time Projection Chamber but for clarity it is shown here attached to the endcap). 
each endcap, and in the small gaps between the HPC modules not already adequately covered for this purpose by the Time of Flight (TOF) scintillators.

The iron return yoke of the magnet is instrumented with limited streamer mode detectors to create a sampling gas calorimeter, the Hadron Calorimeter (HAC). A system to read out the HAC tubes as well as the pads, in order to give a more detailed picture of the hadronic showers and thus better distinction between showers caused by neutral and charged hadrons and better muon identification, was implemented in the barrel part of the detector early in 1995 and is now being implemented also in the endcaps.

Muon identification is achieved by comparing the extrapolations of the reconstructed tracks with the hits in the Barrel (MUB) and Forward (MUF) muon drift chambers. In 1994 a layer of Surrounding Muon Chambers (SMC) [6] based on limited streamer tubes was installed outside the endcaps to fill the gap between the barrel and forward regions.

The Ring Imaging CHerenkov (RICH) detectors provide charged particle identification in both the barrel (BRICH) and forward (FRICH) regions. They combine liquid and gas radiators to identify charged particles over most of the momentum range at LEP1. Though the main structures were installed before startup in 1989, the radiators, fluid systems, chambers and electronics were installed and brought into operation in stages during 1990 to 1993. The BRICH became fully operational during 1992 and the FRICH at the beginning of 1994 . Of the events in Table $1,3320 \mathrm{~K}$ are with the gas radiator of the BRICH fully operational and $2240 \mathrm{~K}$ with both radiators of the BRICH fully operational. The full FRICH detector was operational for $2150 \mathrm{~K}$ events.

\section{Trigger}

The DELPHI trigger system and the determination of the trigger efficiency from the data are described in detail in [7].

In order to cope with high luminosities and large background rates, the trigger system is composed of four successive levels (T1, T2, T3 and T4) of increasing selectivity. The first two trigger levels ( $\mathrm{T} 1$ and $\mathrm{T} 2$ ) are synchronous with respect to the Beam Cross Over signal (BCO). T1 acts as a loose pre-trigger while a positive $\mathrm{T} 2$ decision triggers the acquisition of the data collected by the front-end electronics (see Section 3). With eight bunches of electrons and positrons circulating at equal distances in the machine, the LEP bunch-crossing interval is $11 \mu \mathrm{s}$. The T1 and T2 trigger decisions are taken $3.5 \mu \mathrm{s}$ and $39 \mu$ s after the BCO respectively. The dead-time introduced is then typically $3 \%$, with $2 \%$ due to $\mathrm{T} 1$ and $1 \%$ to $\mathrm{T} 2$ for a typical readout time of $3 \mathrm{~ms}$ per event.

The inputs to $\mathrm{T} 1$ are supplied by individual detectors, namely by the fast tracking detectors (ID, OD, FCA and FCB), by the scintillator arrays in the barrel region (Time Of Flight, TOF) and in the endcaps (Forward HOdoscope, HOF), by the scintillators embedded in the HPC, by the FEMC and by the MUB. In T2 these are complemented by signals from the TPC, HPC and MUF and combinations of signals from different subdetectors are used. T1 and T2 have been active since LEP startup. T3 and T4 are software filters performed asynchronously with respect to the BCO. T3 halves the background passing $\mathrm{T} 2$ by applying the same logic as T2 but using more detailed information. It was implemented in 1992 with the aim of maintaining the data logging rate below $2 \mathrm{~Hz}$. T4 was implemented in 1993 to tag, and in 1994 to reject, about half of the background events remaining after T3.

Each subdetector contributes to the trigger decision with data generated by the respective subtrigger processors. Those with low counting rates produce their own triggers while ones most affected by background are grouped in level 2 majorities, i.e. at least one 
acceptable coincidence of 2 signals out of the $n$ inputs forming the majority is required. This is an efficient way to correlate detectors in a "quasi" single-track or single-cluster configuration that avoids the background typical of single detector triggers. The T1 and T2 decisions are taken by OR-ing a number of "in time" combinations of signals.

The overlapping geometrical acceptance of the different detectors provides substantial redundancy between the different trigger conditions. This feature of the DELPHI trigger ensures high and stable efficiency over long running periods. The trigger signal configuration used in T2 up to 1994 is given in Table 2:

- Track elements give trigger signals in the TPC, FCA/FCB, ID, OD and TOF. A transverse momentum cut $p_{t} \geq 1 \mathrm{GeV} / c$ for $29^{\circ} \leq \theta \leq 151^{\circ}$ (TPC) and $p_{t} \geq 1.6 \mathrm{GeV} / c$ in the forward/backward region (FCA/FCB) $11^{\circ} \leq \theta \leq 33^{\circ}$ and $147^{\circ} \leq \theta \leq 169^{\circ}$ is applied.

- Muons also give trigger signals in the barrel region, $50^{\circ} \leq \theta \leq 130^{\circ}$ with a $1^{\circ}$ hole at $90^{\circ}$, in the MUB and in the forward and backward regions, $15^{\circ} \leq \theta \leq 41^{\circ}$ and $139^{\circ} \leq \theta \leq 165^{\circ}$, in the HOF and in the MUF.

- Electromagnetic energy deposition gives trigger signals in the barrel region in the HPC and in the forward/backward regions in the FEMC. Energy depositions above $2 \mathrm{GeV}$ and $2.5 \mathrm{GeV}$ respectively are demanded. A lower threshold is applied in the FEMC $(1.2 \mathrm{GeV})$ when it is correlated with other detectors.

- Hadronic energy deposition gives trigger signals in the Hadron Calorimeter, both barrel (HAB) and forward (HAF). Energy deposition thresholds of 0.5, 2 and $5 \mathrm{GeV}$ -this last is referred as High Threshold- are used.

The redundancy between the different trigger components also makes it possible to determine both the trigger efficiency and its maximal error with good precision [7]. The global trigger efficiency for electron and muon pairs is consistent with 1 at the level of $10^{-4}$ for polar angles between $20^{\circ}$ and $160^{\circ}$. Even for single tracks, provided their momentum transverse to the beam exceeds $1 \mathrm{GeV} / c$, the efficiencies in the barrel $\left(42^{\circ} \leq \theta \leq 138^{\circ}\right)$ and forward $\left(10^{\circ} \leq \theta \leq 32^{\circ}\right)$ and backward $\left(148^{\circ} \leq \theta \leq 170^{\circ}\right)$ regions exceed $95 \%$. In the barrel region, single photons are triggered by the HPC scintillators and also (at T2) by the charge pattern recorded in the HPC: the single photon efficiency rises linearly from $\sim 5 \%$ for photons between 1 and $2 \mathrm{GeV}$ to $\sim 60 \%$ for photons above $4 \mathrm{GeV}$. Due to their high final state multiplicity, hadronic events $\left(e^{+} e^{-} \rightarrow \mathrm{Z} \rightarrow\right.$ hadrons $)$ are triggered with an efficiency hardly distinguishable from 1 over nearly the full solid angle.

\section{Data Acquisition, Control and Monitoring}

The DELPHI online system performs three basic tasks. The Data Acquisition System (DAS) [8] reads out digitized data from the detector, storing the results for subsequent analysis. The data are also used to monitor detector performance online. Detector operation is monitored and controlled by the Slow Controls system [9].

\subsection{Data Acquisition System}

From the front-end electronics to the central event building, the DELPHI DAS is based on the Fastbus standard. It uses over 150 Fastbus crates and more than 70 embedded microprocessors, connected over an Ethernet network to a VAX cluster.

The DAS is split into "partitions" each corresponding to a subdetector or half a subdetector, as well as one for the trigger system. Each partition uses its own digitization 


\begin{tabular}{|l|c|lc|}
\hline \multicolumn{4}{|c|}{ Luminosity } \\
\hline Trigger & Rate $(\mathrm{Hz})$ & \multicolumn{3}{|c|}{ Detection } \\
& & $e$ & $\mu \quad \gamma \quad c^{ \pm}$ \\
\hline SAT/STIC Bhabha & 0.8 & $\bullet$ & \\
Single arm & 0.4 & $\bullet$ \\
Delayed Bhabha & $\ll 0.1$ & $\bullet$ \\
\hline
\end{tabular}

\begin{tabular}{|c|c|c|c|c|c|}
\hline \multicolumn{6}{|c|}{ Barrel region } \\
\hline \multirow[t]{2}{*}{ Trigger } & \multirow[t]{2}{*}{ Rate $(\mathrm{Hz})$} & \multicolumn{4}{|c|}{ Detection } \\
\hline & & $e$ & $\mu$ & $\gamma$ & $c^{ \pm}$ \\
\hline TPC & 0.8 & $\bullet$ & $\bullet$ & & $\bullet$ \\
\hline $\mathrm{OD}^{*} \mathrm{HPC}$ & 0.2 & $\bullet$ & & - & \\
\hline $\mathrm{OD}^{*} \mathrm{TOF}$ & 0.4 & • & & • & - \\
\hline HPC ${ }^{*} \mathrm{TOF}$ & 0.3 & $\bullet$ & & - & \\
\hline $\mathrm{HPC} \geq 2$ clusters & 0.1 & • & & - & \\
\hline $\mathrm{ID}^{*}(\mathrm{MUB}+\mathrm{HAB})$ & 0.5 & & • & & $\bullet$ \\
\hline HPC (Single- $\gamma$ ) & 0.3 & • & & • & \\
\hline
\end{tabular}

\begin{tabular}{|l|c|cccc|}
\hline \multicolumn{5}{|c|}{ Intermediate region } \\
\hline Trigger & Rate $(\mathrm{Hz})$ & \multicolumn{4}{c|}{ Detection } \\
& & & $\mu^{ \pm}$ & $\gamma$ & $c^{ \pm}$ \\
\hline ID $^{*}(\mathrm{HAF}+\mathrm{MUF})$ & 0.1 & & $\bullet$ & \\
$\mathrm{HPC}^{*}(\mathrm{HAF}+\mathrm{FEMC})$ & 0.1 & $\bullet$ & $\bullet$ & $\bullet$ \\
\hline
\end{tabular}

\begin{tabular}{|l|c|ccc|}
\hline \multicolumn{5}{|c|}{ Forward/Backward region } \\
\hline Trigger & Rate (Hz) & \multicolumn{3}{|c|}{ Detection } \\
& & & $e^{\prime}$ & $\gamma$ \\
\hline TPC*FCA/B & 0.2 & $\bullet$ & $\bullet$ & $\bullet$ \\
MUF*FCA/B & 0.1 & & $\bullet$ & \\
TPC*MUF & 0.1 & & $\bullet$ & \\
FEMC*FCA/B & 0.2 & $\bullet$ & & \\
FCA/B*HAF & 0.4 & & $\bullet$ & $\bullet$ \\
HOF Back-to-back & 0.3 & & & \\
FEMC $\geq 2$ clusters & 0.2 & $\bullet$ & $\bullet$ & \\
FEMC (High Threshold) & 0.5 & $\bullet$ & $\bullet$ \\
\hline
\end{tabular}

\begin{tabular}{|l|c|l|}
\hline \multicolumn{3}{|c|}{ Calibration and special triggers } \\
\hline Trigger & Rate $(\mathrm{Hz})$ & Detection \\
\hline$N I M$ & 0.04 & Random trigger \\
TPC*(SAT/STIC) & 0.1 & Two-photon trigger \\
TOF*HAB (High Threshold) & 0.2 & Search trigger \\
HOF*MUPARAL & 0.1 & Halo- $\mu$ trigger \\
\hline
\end{tabular}

Table 2: List of triggers presently used in T2. The names of the participating subdetectors are indicated together with the rates and the sensitivity to electrons $(e)$, muons $(\mu)$, photons $(\gamma)$ and charged particles $\left(c^{ \pm}\right)$. The logical "OR" and "AND" combinations are shown as "+" and "*", respectively. 
modules, although several partitions standardise their time measurement on the LTD modules [10]. In order to reduce the dead time at each trigger, almost all front-end modules are equipped with a 4-event buffer. Therefore the main task of the DAS on receiving a T2 trigger is to switch to the next buffer, if available. This process is called the Front-End Freeing (FEF) and takes about $3 \mathrm{~ms}$, inducing a dead time of $0.4 \%$. The handling of the trigger and the read-out of the front-end digitizers are performed by the "Crate Processor" software package, consisting of a general skeleton with hooks for detector dependent readout routines and real time configuration, running inside a $16 \mathrm{MHz}$ M68020-based Fastbus master called a FIP (Fastbus Intersegment Processor). The T2 trigger handling is synchronous with the beam crossing while the readout of the front-ends is performed asynchronously.

The readout of each individual partition is performed asynchronously by a "Local Event Supervisor" (LES) software process, also running in a FIP. This software is fully standard, only a few partitions needing a specialised formatting routine. At this level, the data are already formatted as banks in the ZEBRA memory management [11]. Each LES has two buffers: one for local spying, and one for the central readout. The events are first transferred and formatted in the Spy Event Buffer (SEB). They are then copied into the Multi-Event Buffer (MEB) if the detector is being read out centrally and to a dedicated VAX station (one for each subdetector) for monitoring and for standalone tests.

The central readout is controlled by the "Global Event Supervisor" (GES) software process, also running in a FIP. It uses all the messages issued by the LESs to build an event inside a Fastbus memory, the Global Event Buffer (GEB). The actual transfer is done by a Fastbus Block Mover. The full ZEBRA structure of the event is set up in the GEB. Only those events which have been accepted by the third level trigger (T3) are built. They are then transferred to the VAX online cluster by means of a CERN Host Interface (CHI), connected to a VAX mainframe by an optical fibre.

The data flow control and the DAS control run on a VAX cluster containing over 40 nodes, built around a network architecture allowing good separation between the monitoring and control traffic.

Central or partition-monitoring data received on the cluster are stored in a shared buffer handled by the Model Buffer Manager (MBM). Several processes can then act on these data. A Data Logger process writes the data to disk if required. For standalone running, there is one Data Logger for each partition. In the Central Readout, the software trigger T4 is first applied to the events and then specialized Data Loggers are used to write data onto selected streams (see Figure 2). T4 is based on a tailored version of the DELPHI reconstruction program DELANA [12] and rejects all events with no track pointing towards the interaction region and no energy release in the calorimeters.

Before 1995, the disk files were then copied locally onto IBM3480 cartridges. Now they are sent over the FDDI optical link network to a Central Data Recording facility at the CERN computing centre where they are copied onto high capacity tapes (10 GByte Digital Linear Tapes). From there, the offline data analysis farm of DELPHI performs the final reconstruction.

\subsection{DAS Control System}

The DAS is controlled by a programmed state machine (State Management Interface, SMI [13]). SMI handles objects, states of objects, and actions to be performed on these objects. It allows states to be changed on the occurrence of events in other objects. An SMI process runs for each domain to be controlled (e.g. subdetector DAS control and 


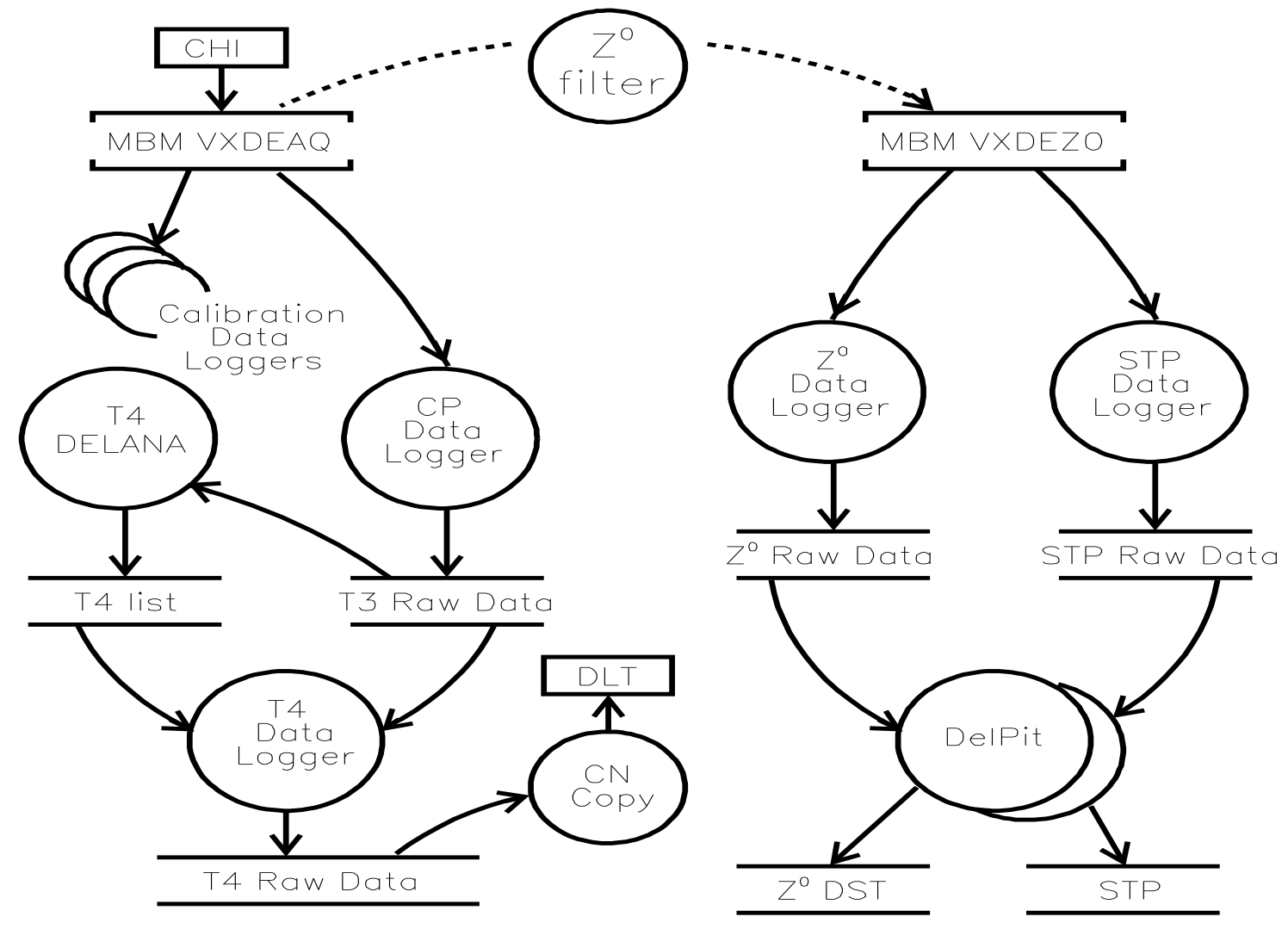

Figure 2: The DELPHI data flow. 
LEP state control). The DAS SMI subsystem has one control unit per partition while a central control unit is used to handle the central readout. The run control is implemented using SMI through a dedicated user interface implemented using the MOTIF standard (DELPHI User Interface, DUI [14]). It allows the operator to reconfigure the system at will, define the data taking conditions, and start and stop data taking.

In order to ease operations, an Autopilot system, also implemented in SMI, can be used to force the system to be permanently in a data taking state. It takes all appropriate actions and asks for intervention only when needed. An even higher level of control, known as "Big-Brother" [15], links the DAS SMI domain to the Slow Controls and to LEP. It detects changes in the LEP machine status to automatically ramp the voltages on the detector up and down and prepare, start and stop the DAS such that data are collected with a maximum efficiency but only while LEP and the detector are in good data taking conditions.

All the DAS processes on the online cluster (including the SMI processes) communicate over a general system based on the server-client paradigm, called the Distributed Information Manager (DIM) [16]. Using this system, servers publish services (e.g. object states and detector high voltages) which can be subscribed to by any client. Currently the DELPHI online DIM system publishes over 15000 services from 300 servers.

\subsection{Slow Controls System}

The Slow Controls system [9] controls the operation of the detector, reporting and (where necessary) acting on significant changes in the detector or its environment, recording such changes where required for the data analysis, and maintaining the safety of the equipment.

Most front-end monitoring and control of temperatures, low voltages, fastbus power supplies, etc., is performed by digital monitoring, relay, and ADC cards in 90 G64 [17] microcomputers. Most high voltages are supplied by the intelligent CAEN [18] system, controlled by G64. Each G64 accepts commands from, and reports significant changes to, the VAX "Elementary Process" responsible for the subsystem. In most cases, standard, configurable, G64 and Elementary Process programs are used. The operator is alerted to problems via the Error Message Utility (EMU [19]) and SMI. Communications [20] between the Elementary Processes and the G64s, database server, and EMU use the Remote Procedure Call model [21], DECnet and OSI transport protocols, running over Ethernet.

Operator and automatic control is effected using SMI. For potentially dangerous conditions, automatic actions implemented in SMI complement hardware interlocks.

The high voltages are raised at the start of a fill in order to take data, and (for many parts of the detector) lowered at the end of a fill to prevent damage from high currents during refilling. These actions are performed under SMI control, either automatically (Big Brother) or at the instigation of the operator.

During data-taking, significant changes in parameters required for the subsequent data analysis (such as chamber pressures, temperatures, voltage values or the operational status of any subdetector or subdetector module) are recorded on the central database [22] by the Elementary Process using a dedicated server process, which sends a copy of all updates to the offline data analysis farm for use by the analysis program.

This system has allowed a single operator to oversee 12609 detector monitoring and control channels. Dead-time incurred from the control system to initiate high voltage 
ramping at the start of fill is normally negligible. High voltage trips and many similar causes of data loss can usually be dealt with rapidly, often (where safe) automatically.

\subsection{Monitoring}

Three stages of data quality monitoring have been implemented: local partition monitoring, central online monitoring (for all events and selected $Z$ events), and online monitoring via event reconstruction (DelPit).

The first two systems are implemented as low priority MBM consumers. They produce histograms which allow the checking of the technical behaviour of each component of the detector. The DelPit monitoring uses a sample of selected events (Z's and selected two prong events) on which the standard reconstruction program of DELPHI (DELANA, see Section 6) is run in situ at the pit. It produces histograms and Ntuples [23] checking more detailed potential problems in the detectors (e.g. mismatches of tracks between detectors and drift velocity variations). One of the operators running DELPHI is in charge of checking these histograms using a dedicated DELPHI Histogram Presenter. A special World Wide Web server is used to keep the histogram descriptions updated and to keep track of all problems encountered.

\subsection{Performance}

The DAS system currently runs with 20 partitions (corresponding to 16 subdetectors, including the trigger system), 14 of which participate in the T3 decision. The average $\mathrm{Z}$ event size is about 150 kbytes. With a T2 rate of about $5 \mathrm{~Hz}$, the rejection power of T3 (which depends on the machine background conditions) is about 1.5, while T4 adds a further factor 1.5 giving a final data recording rate of about $2 \mathrm{~Hz}$. Typically the data sample comprises $15 \% \mathrm{Z}$ decays, $30 \%$ Bhabha events and $55 \% \gamma \gamma$, cosmics and background events (at the $Z$ peak energy).

As a typical example, the total dead time of DELPHI integrated over the whole 1995 scanning period is $14.4 \%$ with the following breakdown: $1.5 \%$ for initial setting up of the detectors at the beginning of each fill, $1 \%$ for DAS internal checking, $2 \%$ for DAS stopped due to the LEP machine background being too high, $7 \%$ for DAS stopped due to DAS crashes, and $3 \%$ due to $\mathrm{T} 1$ and $\mathrm{T} 2$ dead time.

\section{Luminosity Measurement}

At $e^{+} e^{-}$colliders, luminosity is measured by counting the number of events of a process with a clear experimental signature, with high statistics and with a cross section which can be calculated theoretically with high precision. The process chosen is $e^{+} e^{-} \rightarrow e^{+} e^{-}$ Bhabha scattering at small angles, which proceeds almost entirely through the exchange of a photon in the $t$-channel.

In DELPHI, before 1994 the absolute luminosity was measured using the SAT detector (acceptance between 43 and $135 \mathrm{mrad}$ in $\theta$ ) [24,25] and the VSAT detector was used to measure the relative luminosities at different energies [26]. In 1994 the SAT was replaced by a new calorimeter, built with the "Shashlik" technique [5], the STIC. The STIC is a sampling lead-scintillator calorimeter formed by two cylindrical detectors placed on either side of the DELPHI interaction region at a distance of $2200 \mathrm{~mm}$, and covers a wider angular region between 29 and $185 \mathrm{mrad}$ in $\theta$ (from 65 to $420 \mathrm{~mm}$ in radius). The blue light produced in the scintillator is read by wavelength shifting fibres placed 
perpendicularly to the scintillator planes. The geometry is quasi-projective, to avoid channeling of the incoming particles through the fibres. The total length of the detector is 27 radiation lengths. Each STIC arm is divided into 10 rings and 16 sectors, giving an $R \phi$ segmentation of $3 \mathrm{~cm} \times 22.5^{\circ}$.

Test beam measurements, repeated with three different STIC modules in 1993, gave an energy resolution of $\sigma_{E} / E=(0.0152 \pm 0.0002) \oplus(0.135 \pm 0.001) / \sqrt{E}(E$ in $\mathrm{GeV})$, a deviation from energy linearity below $\pm 1 \%$ and an energy deposition for muons 4 standard deviations above the pedestal. At $45.6 \mathrm{GeV}$ the energy resolution is $\sigma_{E} / E=2.7 \%$, which is in good agreement with the test beam measurement.

\subsection{The Luminosity Analysis}

Bhabha events are selected on the basis of the energy clusters in the calorimeters. Clusters are made by joining together neighbouring towers with at least $60 \mathrm{MeV}$ deposited energy. More than one cluster is allowed in each STIC arm in order to take into account radiative Bhabha events. For the Bhabha selection only the most energetic cluster of each STIC arm is used. The radial coordinate of the shower is reconstructed from the sharing of the energy deposited in the rings above and below the one with the largest energy deposition, using a parametrisation extracted from the test beam data and corrected using the data collected at LEP in 1994.

\subsubsection{Definition of the Acceptance}

Radial fiducial cuts are applied, requiring one cluster to lie within a "tight" acceptance and that on the opposite side to be within a more loosely defined acceptance to reduce the dependence of the visible cross section on displacements of the $e^{+} e^{-}$interaction point.

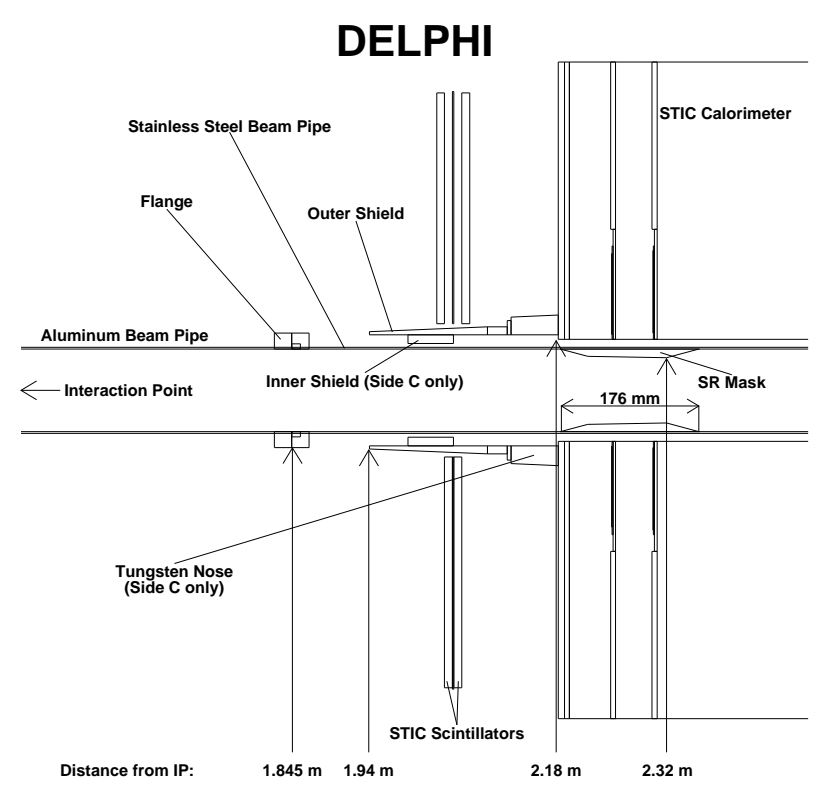

Figure 3: Layout of the STIC region.

For the STIC an accuracy of $0.1 \%$ in the luminosity measurement requires control of biases in the radial position at the inner edge of the acceptance to better than $50 \mu \mathrm{m}$. The minimum angle of the tight acceptance region, which for the STIC is $43.5 \mathrm{mrad}$, 
is defined by a 17 radiation length tungsten ring, called the "nose", placed in front of one arm (in the so-called "Side C"). Its outer surface points to the nominal interaction point (see Figure 3) and was machined to an accuracy of $13 \mu \mathrm{m}$. A cut on the shower energy detected in the masked calorimeter $\left(E_{\text {Masked }}>65 \% E_{\text {Beam }}\right)$ therefore translates into a very sharp radial cut: the root mean square width of the transition region, as determined in a testbeam using a microstrip telescope to define the trajectory of the incoming particle, is $\simeq 25 \mu \mathrm{m}$.

The other boundaries of the acceptance are defined using the radius $R$ reconstructed by the calorimeter. The outer edge of the tight acceptance region is at $R=28 \mathrm{~cm}$. The looser acceptance in the other (unmasked) calorimeter lies between $R=7 \mathrm{~cm}$ and $R=31 \mathrm{~cm}$. As the tight acceptance is defined using the tungsten ring, it is not possible to alternate the tight and loose acceptance sides from event to event. This leads to a first order dependence of the accepted cross section on the longitudinal position of the interaction point. The effect is corrected through the reconstruction of the position of the interaction point. This turns to be the largest source of systematic uncertainty in the present measurement of the luminosity.

\subsubsection{Background Rejection}

The main contamination of the Bhabha signal is from spurious coincidences of offmomentum beam particles. It is severely suppressed by requiring on both arms that the most energetic cluster has an energy above $65 \%$ of the beam energy (see Figures 4 and 5). An acoplanarity cut is also applied, requiring the azimuthal difference $\Delta \phi$ between the two clusters to be $160^{\circ}<\Delta \phi<200^{\circ}$. The STIC can monitor the rate of accidental coincidences by using a special "delayed Bhabha" trigger. This trigger uses the same thresholds and geometry as the Bhabha trigger but it fires if there is a coincidence between a single arm trigger in one arm and a single arm trigger taken in the opposite calorimeter just one LEP cycle before. The probability of such an event is the same as that of a fake coincidence generated by off-momentum electrons. These events are not passed through the usual Bhabha selection as only the information of the later event is kept in the calorimeter. The overall rate of these events is used to set an upper limit to the background rate, which was found to be a factor $10^{-4}$ lower than the Bhabha rate during 1994.

\subsection{Results}

The integrated luminosity is given by $N_{B h a b h a} / \sigma_{v i s}$ where $N_{B h a b h a}$ is the number of events selected by the luminosity cuts after background subtraction and $\sigma_{v i s}$ is the accepted cross section calculated using the BHLUMI generator [27]. The correction for the missing s-channel Z exchange contribution evaluated using the BABAMC generator [28] is $+0.12 \%$. The main sources of experimental uncertainty are as follows:

- $0.06 \%$ due to the uncertainty in the fill by fill determination of the interaction point with respect to the STIC position;

- $0.04 \%$ due to the uncertainty in the inner radius cut determined using the tungsten mask;

- the systematic uncertainty due to the different efficiencies of the other cuts for LEP data and for simulation $(0.03 \%$ for the energy cut, $0.02 \%$ for the outer radii cuts, $0.02 \%$ for the inner radius cut on the side opposite the mask, and $0.01 \%$ for the acoplanarity cut); 


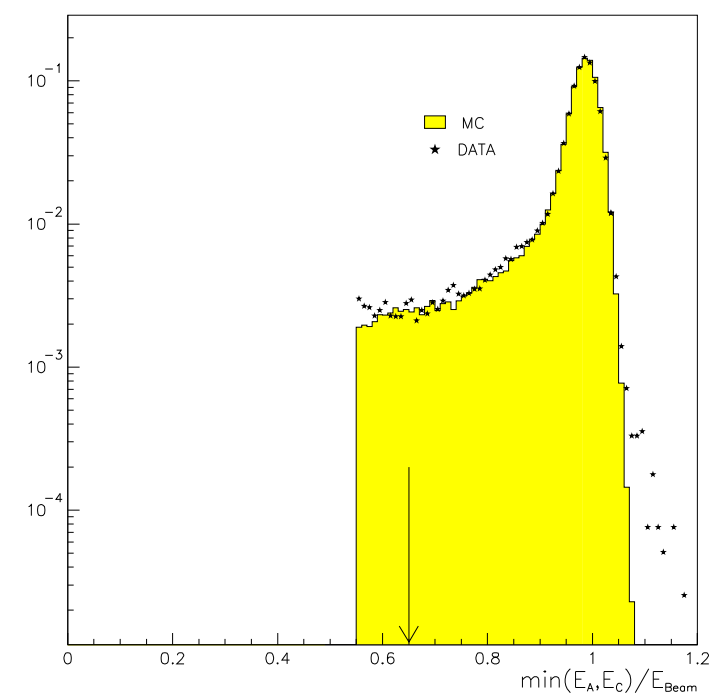

Figure 4: Distribution obtained by selecting the highest energy cluster in each of the two STIC arms and plotting the lower one.

- $0.02 \%$ due to the uncertainty in the subtraction of background due to off-momentum particles;

- $0.02 \%$ due to the uncertainty in the determination of the trigger efficiency.

Other sources such as the thermal expansion of the structure supporting the calorimeters are continuously monitored online by means of position and temperature probes. Their global effect on the systematic error turns out to be only about $0.01 \%$. The stability of the noise and of the gain of the electronic read out chain was monitored online and cross-checked offline throughout the year. Thus the overall experimental precision of the luminosity measurement for 1994 data was $0.09 \%$.

\section{Detector Simulation}

The primary aim of the simulation program is to produce data which are as close as possible to the real raw data. These data are then treated by the reconstruction and analysis programs in exactly the same way as the real data. This models the detailed response of both the complete detector and the data analysis chain to a particular physics process. The DELPHI Simulation, DELSIM [29], is based on three components which can be summarized as follows:

- a model for the generation of the primary physics process: in most cases, this is provided by external programs, like JETSET [30], HERWIG [31], and ARIADNE [32] for production of quark final states $e^{+} e^{-} \rightarrow q \bar{q}$, DYMU3 [33] for $e^{+} e^{-} \rightarrow \mu^{+} \mu^{-}$, BABAMC [28] for $e^{+} e^{-} \rightarrow e^{+} e^{-}$, KORALZ [34] for $e^{+} e^{-} \rightarrow \tau^{+} \tau^{-}$.

- the general part for following particles through the DELPHI detector until they hit an active detector component: this is done by stepping through the magnetic field and includes the possibility that the particles give rise to secondary interactions. 


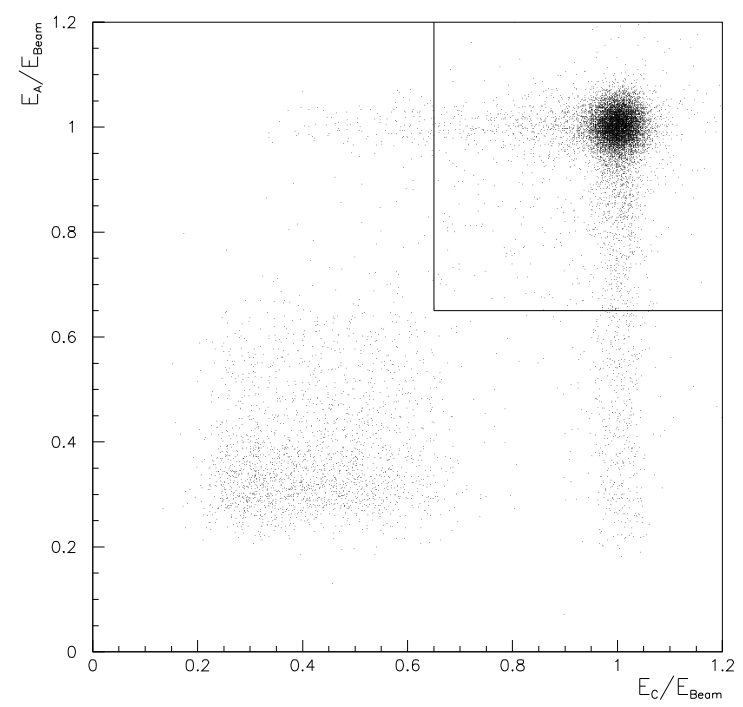

Figure 5: Distribution of the highest energy cluster of one STIC arm versus the highest energy cluster of the other; the region of the cut is indicated by the line.

- following particles inside the active detector components and the realistic simulation of the detector response: this part is specific to every detector component and the modularity of the code is such that each detector component corresponds to an independent software module.

From time to time the generators for $q \bar{q}$ final states are retuned using the LEP data (see for example [35]) and the information on $b$ and $c$ hadron decays is updated to account for the new experimental measurements.

Event generators other than the ones mentioned above are also used. The requirement is that they produce an output in the same format as the particle common from JETSET. This is transformed by DELSIM into a standard event structure, based on the ZEBRA memory management package [11], which is used by all the software modules of DELSIM.

The particle trajectories are followed by stepping through the magnetic field. The distance that a particle can traverse without change of material varies enormously from one part of the detector to another. It is therefore crucial to optimize the step size to the thickness of the material components in order to avoid wasting CPU time. The optimal step size inside a given material is calculated by a package of routines (DDAPP) [36] which use information from the DELPHI detector Data Base (CARGO) [37], which contains a very detailed description of the material layers and of the sensitive components of the detector.

Particle tracking includes energy loss, multiple scattering and the following secondary processes: photoelectric effect, emission of delta rays, bremsstrahlung, annihilation of positrons, pair production, Compton scattering, weak decays and nuclear interactions (this last using GEANTH, the simulation of hadronic interactions inside GEANT [38]). The material parameters which determine the rates of the above processes are also extracted from the CARGO data base.

As soon as a particle enters an active detector component, control of the track following is given to the corresponding software module. Most modules follow the particles by 
using tools provided by the general routines outlined above. Some modules use different methods (for example the HPC simulation, which needs a very accurate description of electromagnetic effects, is based on EGS4 [39]). When a particle crosses the sensitive volume of a detector, the relevant information is stored to compute the detector response in the form of electronics signals as for real data. The backgrounds and efficiencies of the sensing devices are also obtained from the CARGO data base. When the particle leaves the detector component or is stopped inside, its parameters and possibly the parameters of accompanying secondary products are transmitted to the general routines which take back the control of the track following.

Checks are performed to verify the simulation by comparing the parameters of individual particles at the entry and the exit of the detector components and by accumulating statistics on the energy loss and secondary processes occurring inside the detector.

In addition to this detailed simulation a simplified version, called FASTSIM [40], has been implemented to obtain a much faster simulation of the DELPHI events. This version uses a simplified description of the material in the detector and calls detector modules which simulate directly the results of the pattern recognition, thus avoiding the time consuming step of producing the raw data.

\section{Reconstruction Program}

The DELPHI Reconstruction program (DELANA [12]) has a highly modular structure to facilitate development of reconstruction code for the individual subdetectors. The backbone of DELANA is the Track ANAlysis and GRAphics package TANAGRA [41] which provides a well defined data structure for storing track and vertex information in a format independent of the various program modules. All data transfer between modules is performed through calls to TANAGRA routines which protect against overwriting of data from one module by another.

Constants required by DELANA (pedestals, distortion corrections, machine running conditions etc.) are obtained from CARGO. At the start of reconstruction of each event, the range of validity dates and times for each block of information is checked against the time stamp of the event and updated if necessary.

At a first stage of the reconstruction program, the code of each subdetector, working independently, decodes the raw data, applies calibrations and where possible performs a local pattern recognition. The output of this stage is a set of track elements. These can be single two dimensional points in $R \phi$ or $R z$ as for the VD, energy clusters from the calorimeters, or fully reconstructed track segments as for the TPC. These track elements provide the input to the global track search and fit processors.

The main track search algorithm used in the barrel region starts with the track segment seen in the TPC and extrapolates inwards and outwards to form candidate strings of track elements with the ID and OD. A second algorithm, used only in the dead zones of the TPC (see later), directly connects track elements in the ID and OD in order to recuperate low curvature tracks that would otherwise be lost. Algorithms are under development to use the combination of the VD and ID or VD and TPC track elements as a pivot for track searching.

In the forward region, the TPC can only be used as pivot detector for polar angles where it can give a well reconstructed track segment. Additional algorithms are therefore needed in this region. Under polar inversion [42] in the plane perpendicular to the magnetic field, a helical trajectory is transformed into a straight line. Search algorithms based on this property are used to form candidate strings of track elements. A third technique, 
particularly important at very small polar angles, uses the track elements measured in FCB and the beamspot to define search roads.

All strings of track elements found by the above search techniques are passed through the full track fitting processor and any remaining ambiguities resolved. The track fit processor is based on Kalman filter techniques [43] and accounts for multiple scattering and energy loss in the material between the measurements.

The fitted tracks are then extrapolated through the detector. It is at this stage that the VD hits are currently associated to the tracks. In addition, a second stage of local pattern recognition in the tracking detectors is performed, using the extrapolations for guidance, allowing the addition of information from a given detector to the track. The tracks are then re-fitted before a final pass of the search algorithms is made over those elements not yet included in a fitted track.

The second stage calorimeter processors associate clusters of energy to the reconstructed charged particle tracks and create neutral tracks from the remaining clusters. Hits in the Muon Chambers are associated to tracks for muon identification and the data of the Ring Imaging Cherenkov Counters treated.

During event reconstruction, information on the status of each subdetector provided by the online monitoring of operation ("Slow Controls" system, see section 3.3) and internal information on decoding problems with the data are combined to give status flags for the various modules. These flags form the basis of run selection files used in the physics analysis.

After reconstruction, a simple filter is used to select interesting triggers for output. Physics analysis teams can supply more sophisticated code to select events in particular physics channels. The results of these tags are used to direct the event to the various output streams defined for a given processing. Normally some $40 \%$ of triggers are retained on the Data Summary Tapes (DST). At this stage, the average size of a hadronic event is 60 kbytes. The "full" DST produced contains detailed information allowing, for example, a) tracks not reconstructed by the standard procedure (e.g. tracks of $\Sigma^{ \pm}, \Xi^{ \pm}$or $\Omega^{ \pm}$that decay before the TPC and of particles that interact before it) to be reconstructed at DST level using different track search algorithms, b) the refitting of previously-found tracks using different alignment constants, distortion corrections or error estimates, and c) the rerunning of much of the electron, muon and charged hadron identification software with improved procedures or constants.

This "DSTFIX" possibility allows a) the quality of the real data to be improved without reprocessing the raw data $a b$ initio, and b) the efficiency, cleanliness and precision of the simulated data to be adjusted to match better in detail the efficiency, cleanliness and precision actually achieved for the real data in any given processing. Routinely, a "short" DST is produced by using this detailed information and then discarding it; a "mini" DST is then produced by compacting the information needed for the majority of physics analyses. The average size of a hadronic event is 20 kbytes at the short DST level and 6 kbytes at the mini DST level.

\section{Tracking Performance}

The tracking system is segmented into a relatively large number of independent tracking devices. In the following, the performance achieved by each of these is briefly reviewed, followed by a brief survey of the results obtained from the combined tracking system. Further details of the performance of the tracking system for $\mathrm{V}^{0}$ reconstruction 
and for vertex reconstruction, impact parameter measurement and $b$-tagging are covered in sections 8 and 9 .

\subsection{Vertex Detector}

The VD consists of three coaxial cylindrical layers of AC coupled silicon strip detectors at average radii of $6.3,9.0$ and $10.9 \mathrm{~cm}$. Each layer covers the full azimuthal angle in 24 sectors with overlaps between adjacent sectors. There are 4 detectors along the beam direction in each sector. For polar angles of $44^{\circ} \leq \theta \leq 136^{\circ}$, a particle crosses all three layers of the VD. The readout pitch is $50 \mu \mathrm{m}$ in the $R \phi$ plane perpendicular to the beam direction.

At the start of 1994, the first (Closer) and third (Outer) layers were equipped with double-sided silicon detectors, having strips orthogonal to each other on opposite sides of the detector wafer, giving measurements also in the $z$ direction. Routing of signals from the $z$ strips to the end of the detector modules is done with a second metal layer on the detector surface, thus keeping the material in the sensitive area to a minimum. The polar angle coverage of the Closer layer was increased to $25^{\circ} \leq \theta \leq 155^{\circ}$. For the $z$ coordinate in the Closer layer, the readout pitch of $49.5 \mu \mathrm{m}$ used near $\theta=90^{\circ}$ is increased to 99 and $150 \mu \mathrm{m}$ for larger $|z|$ values in order to optimise the number of electronic channels. Similarly, the pitch values for the Outer layer are 42 and $84 \mu \mathrm{m}$.

The alignment of the VD uses particle tracks from $Z$ decays, taking as its starting point the results of a mechanical survey. The procedure uses hadron tracks which pass through the overlap regions between sectors, isolated hadron tracks with 3 hits contained within a sector, and tracks from $\mathrm{Z} \rightarrow \mu^{+} \mu^{-}$decays ("dimuon" events). Only the momenta of the hadrons are taken from the measurements of other detectors. Tracks in the overlaps are used to refine the $R \phi$ rotations and translations of the modules in a layer. The tracks in dimuon events and the 3 -hit tracks constrain the relative positions of modules in different layers. A similar procedure using overlap and dimuon tracks is used for the $z$ alignment.

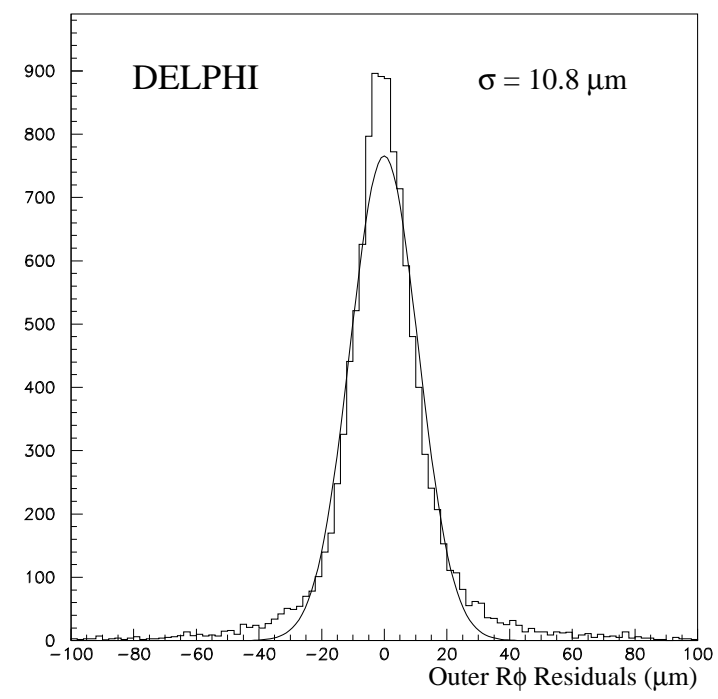

Figure 6: Vertex detector residual distribution in the $R \phi$ plane from tracks passing through the overlap regions in the Outer layer. 


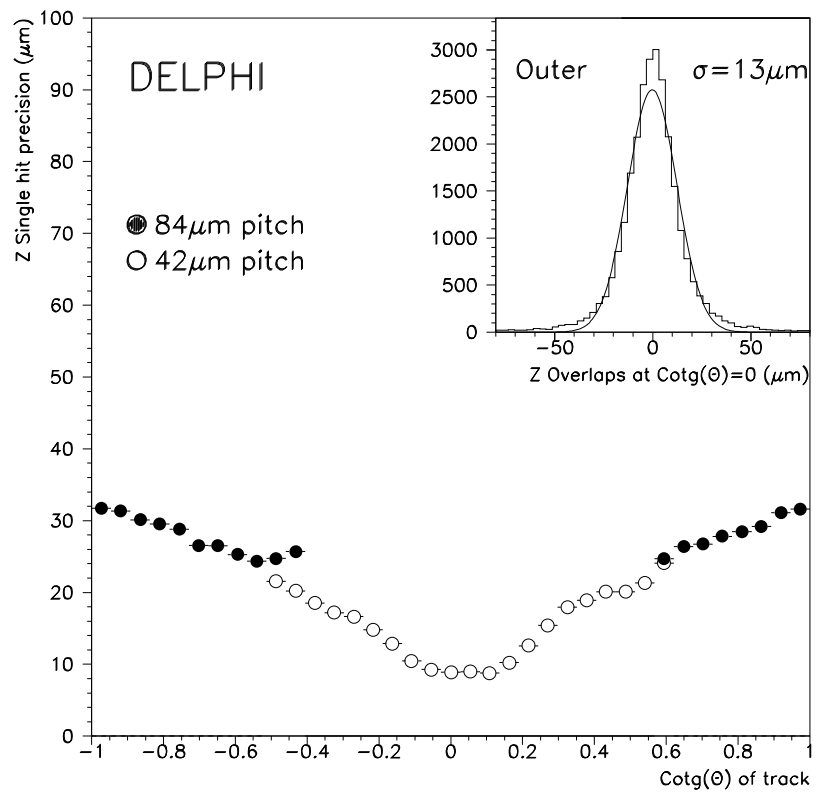

Figure 7: Vertex detector $z$ hit precision as a function of the track incidence angle. The inset shows the $z$ residual distribution for normal incidence.

The single hit precision of the detector can be estimated from the residual distributions of hits left out from the fit in the overlap regions. Such distributions include contributions from remaining alignment uncertainties. Figure 6 shows the $R \phi$ residual distribution averaged over all sectors and track incidence angles. The width of the central Gaussian corresponds to a single hit precision of $7.6 \mu \mathrm{m}$ for one layer. The non-Gaussian tails are due to different cluster characteristics (size, pulse height, noise) and incidence angles (see discussion in [2]) and they are well understood: for example the single track $\chi^{2}$ probability distribution is very flat with only $6 \%$ of tracks having probabilities below $1 \%$.

The single hit precision of the $z$ coordinate is a function of the incidence angle of the track as shown in Figure 7 , reaching a value of $9 \mu \mathrm{m}$ for tracks perpendicular to the modules. The width of the distribution in the inset must be divided by $\sqrt{2}$ to obtain the single hit precision. More detailed descriptions of the vertex detector and its performance can be found in references $[2,3]$.

\subsection{Inner Detector}

The inner drift chamber of the ID has a jet-chamber geometry with 24 azimuthal sectors, each providing up to $24 R \phi$ points per track between radii of 12 and $23 \mathrm{~cm}$. For polar angles in the range $23^{\circ} \leq \theta \leq 157^{\circ}$, a track crosses a volume of the detector sensed by a minimum of 10 wires.

Surrounding the jet-chamber, there are 5 cylindrical MWPC layers with sense wires spaced by about $8 \mathrm{~mm}$ (192 wires per layer) and with circular cathode strips giving $R z$ information. The $R \phi$ measurements are mainly used in triggering, but also provide the possibility of resolving the left/right drift ambiguities inherent in the jet-chamber. The polar angle coverage is $30^{\circ} \leq \theta \leq 150^{\circ}$.

In the jet chamber, the drift field strength, gas pressure and temperature are monitored continuously and used to correct calibration constants measured before installation of the detector. Residual calibration errors are corrected at the level of the reconstructed local 
track elements, using tracks from $\mu^{+} \mu^{-}$and $e^{+} e^{-}$events constructed from the $3 R \phi$ measurements in the VD and the transverse momentum. Deviations of the measured coordinates from the extrapolations of these tracks to the ID are parametrised as a function of $\phi$ and used to correct the local track element parameters.

Single wire precisions vary from $75 \mu \mathrm{m}$ to $125 \mu \mathrm{m}$ depending on the drift distance. After correction, the precisions of the parameters of the local track element in $\mathrm{Z} \rightarrow \mu^{+} \mu^{-}$ events are $\sigma(R \phi)=50 \mu \mathrm{m}$ and $\sigma(\phi)=1.5 \mathrm{mrad}$. The two track resolution is about $1 \mathrm{~mm}$. The $z$ precision from a single MWPC layer for an isolated track varies from 0.5 to $1 \mathrm{~mm}$ depending on $\theta$.

Since the beginning of 1995 a new longer ID has been operational. The inner drift chamber has exactly the same wire configuration as the previous one. The polar angle acceptance for tracks giving a hit on the 10 innermost anode wires is now $15^{\circ} \leq \theta \leq 165^{\circ}$. First results indicate an average single wire precision of $85 \mu \mathrm{m}$ and local track element precisions of $\sigma(R \phi)=40 \mu \mathrm{m}$ and $\sigma(\phi)=0.89 \mathrm{mrad}$. Surrounding the jet chamber there are now 5 cylindrical layers of straw tube detectors (192 tubes per layer) measuring $R \phi$ and having the same functionality as the old MWPC trigger layers. There is no longer any $z$ measurement. The polar angle acceptance is also $15^{\circ} \leq \theta \leq 165^{\circ}$. The additional measurement of the drift time in each tube to improve the $R \phi$ measurement is under development.

\subsection{Time Projection Chamber}

Both end-plates of the TPC are divided into 6 azimuthal sectors, each with 192 sense wires and 16 circular pad rows with constant spacing. The detector thus provides up to 16 space points per particle trajectory at radii of 40 to $110 \mathrm{~cm}$ between polar angles of $39^{\circ} \leq \theta \leq 141^{\circ}$. At least three pad rows are crossed down to polar angles of $20^{\circ} \leq \theta \leq$ $160^{\circ}$. The dead space between the pads of adjacent end-plate sectors corresponds to $4 \%$ of the $R \phi$ plane. The characteristics and running conditions of the DELPHI TPC are summarised in Table 3.

\begin{tabular}{|l|l|}
\hline \multicolumn{2}{|c|}{ DELPHI TPC characteristics } \\
\hline sense wires spacing & $4 \mathrm{~mm}$ \\
sense wires diameter & $20 \mu \mathrm{m}$ \\
sense wires high voltage & $1435 \mathrm{~V}$ \\
maximum drift length & $1.34 \mathrm{~m}$ \\
magnetic field & $1.23 \mathrm{~T}$ \\
drift field & $187 \mathrm{~V} \mathrm{~cm}$ \\
drift speed of primary electrons & $7 \mathrm{~cm} \mu \mathrm{s}^{-1}$ \\
gas pressure & stabilized to $1 \mathrm{~atm}_{\text {gas temperature }}$ \\
gas mixture & $80 \% \mathrm{Cr} 20 \% \mathrm{CH}_{4}$ \\
\hline
\end{tabular}

Table 3: Characteristics and running condition of the DELPHI TPC in 1993-1995.

Laser tracks are used to monitor the drift velocity continuously during data taking. The relative precision of the drift velocity measurement is better than $2 \times 10^{-4}$. The drift velocity calibration is checked from the difference in $z$ of the two vertices formed separately from tracks in the $z$-positive and $z$-negative halves of the TPC. 
Distortions in the $R \phi$ and $R z$ planes are determined by extrapolating tracks from $\mathrm{Z} \rightarrow \mu^{+} \mu^{-}$decays from the VD to the TPC pad rows. The distortions are parametrised from the distances between reconstructed and extrapolated points. In 1994, each muon track was separately extrapolated from the two $R z$ hits in the VD, while for previous years the $z$ information of the cathode strips in the MWPC layers of the ID was used while treating the $\mu^{+}$and $\mu^{-}$as a single track.

The single point precision for tracks from $\mathrm{Z} \rightarrow \mu^{+} \mu^{-}$decays is $250 \mu \mathrm{m}$ in the $R \phi$ plane and $880 \mu \mathrm{m}$ in the $R z$ plane. The two-point resolution is about $1 \mathrm{~cm}$ in both directions. Distortions currently limit the precision on the track elements to about $150 \mu \mathrm{m}$ in $R \phi$ and about $600 \mu \mathrm{m}$ in $z$.

\subsection{Outer Detector}

The OD consists of 5 layers of drift tubes, operated in the limited streamer mode, located between radii of 197 and $206 \mathrm{~cm}$. Successive layers are staggered and adjacent modules of the 24 azimuthal sectors overlap, giving full azimuthal coverage. Three layers are equipped to read the $z$ coordinate by timing the signals at the ends of the anode wires. The active length of the detector corresponds to polar angles of $42^{\circ} \leq \theta \leq 138^{\circ}$.

Individual pedestals for each channel are obtained by injection of a test pulse, while the global timing of the detector is adjusted to give the best fit of the local track element as the common tangent to the drift circles in each OD layer. The residuals of this fit indicate a single point precision of $\sigma(R \phi)=110 \mu \mathrm{m}$, independent of the drift distance, with a single cell efficiency above $99.5 \%$. The precision in the $z$ coordinate is $\sigma(z)=3.5$ $\mathrm{cm}$.

\subsection{Forward Chamber A}

Three modules of FCA are mounted on each end of the TPC at a distance from the interaction point of about $160 \mathrm{~cm}$ in $|z|$. A module consists of 2 staggered planes of drift tubes, operated in the limited streamer mode. There is a rotation of $120^{\circ}$ between the wire orientations of the modules. The chamber covers polar angles of $11^{\circ} \leq \theta \leq 32^{\circ}$ and $148^{\circ} \leq \theta \leq 169^{\circ}$.

The non-linear drift time/distance relation was determined in a test beam, where single wire residuals had an average root mean square of $190 \mu \mathrm{m}$, being worse near the sense wires and in the corners of the drift tubes. In normal conditions, where the direction of the particle is not known a priori, the reconstructed track elements have precisions of $\sigma(x)=290 \mu \mathrm{m}, \sigma(y)=240 \mu \mathrm{m}, \sigma(\theta)=8.5 \mathrm{mrad}$, and $\sigma(\phi)$ averaged over $\theta$ is $24 \mathrm{mrad}$.

\subsection{Forward Chamber B}

FCB is a drift chamber at an average distance of $|z|=275 \mathrm{~cm}$ from the interaction point. The chamber consists of 12 readout planes, coordinates in each of three directions rotated by $120^{\circ}$ being defined by 4 planes. The sensitive area of the chamber corresponds to polar angles of $11^{\circ} \leq \theta \leq 36^{\circ}$ and $144^{\circ} \leq \theta \leq 169^{\circ}$.

Internal calibration of the chamber (in particular of the drift time/distance relation) is performed using the muons in the LEP beam halo. Single wire residuals show a small dependence on the distance from the sense wire and have an average value of $300 \mu \mathrm{m}$. The precisions achieved on the parameters of the reconstructed track elements are $\sigma(x, y)=150 \mu \mathrm{m}, \sigma(\theta)=3.5 \mathrm{mrad}$ and $\sigma(\phi)=4.0 / \sin \theta \mathrm{mrad}$. 


\subsection{Global Alignment}

At LEP1 the global alignment of the tracking chambers is performed mainly using $\mathrm{Z} \rightarrow \mu^{+} \mu^{-}$events in order to exploit the constraint on the momentum derived from the beam energy. To align the barrel detectors, the OD is chosen as reference since the wire positions are known to a precision of $30 \mu \mathrm{m}$ from optical and mechanical surveys and the detector has a good time stability and a long lever arm with respect to the interaction point. The position of the VD with respect to the OD is determined assuming the two muons form a single track ${ }^{\dagger}$. Then the ID and TPC are aligned using the reference tracks formed by the VD and OD, imposing a fixed momentum but relaxing the collinearity constraint.

After correction for distortions in the individual barrel detectors, muon tracks reconstructed in the TPC are extrapolated to the forward region, and the forward chambers (FCA and $\mathrm{FCB}$ ) are aligned.

After aligning the full tracking system, tracks from the dimuon sample are again extrapolated through the detector to align in $z$ the barrel electromagnetic calorimeter (HPC) and determine the positions of the mirrors and drift-tubes of the Ring Imaging Cherenkov Counters.

\subsection{Momentum Precision}

The momentum precision of the tracking system in the barrel region is illustrated in Figure $8(\mathrm{a})$, which shows the measured inverse momenta of muons from $Z \rightarrow \mu^{+} \mu^{-}$events in which the acollinearity of the two muons is below $0.15^{\circ}$ (to remove radiative $\mathrm{Z}$ decays) and whose tracks contain information from all the barrel detectors (VD, ID, TPC, OD). The distribution can be fitted to the sum of two Gaussians. A width of

$$
\sigma(1 / p)=0.57 \times 10^{-3}(\mathrm{GeV} / c)^{-1}
$$

is obtained for the narrower Gaussian. The tails of the distribution require the wider Gaussian. This has a peak value of about $8 \%$ with respect to the total peak, and a width of $1.04 \times 10^{-3}(\mathrm{GeV} / \mathrm{c})^{-1}$.

A similar plot for muons in the forward region seen in at least the Closer layer of the $\mathrm{VD}$ and in FCB is shown in Figure 8(b), where a precision of

$$
\sigma(1 / p)=1.31 \times 10^{-3}(\mathrm{GeV} / c)^{-1}
$$

is measured. Table 4 summarises the momentum precision for dimuons in different polar angle regions, and with different combinations of tracking detectors included in the fitted track.

The precisions obtained on the track parameters at other momenta can be estimated by comparing the simulated and reconstructed parameters in a sample of generated $\mathrm{Z}$ hadronic decays. Figure 9(a) shows the behavior of the $\sigma$ of the distribution of the difference between the reconstructed and simulated momenta as a function of the polar angle $\theta$, for samples of tracks in different momentum intervals. As can be seen, the precision remains essentially constant over the barrel region for a given momentum but deteriorates in the forward regions of the detector. The variation of the average momentum precision for tracks in the barrel region as a function of momentum is shown in Figure 9(b). Analogous plots for the precision in the azimuthal angle $\phi$ are shown in Figures $9(\mathrm{c})$ and $9(\mathrm{~d})$ while those for the polar angle $\theta$ are shown in Figures $9(\mathrm{e})$ and $9(\mathrm{f})$.

\footnotetext{
${ }^{\dagger}$ For 1994 data the effect of beam acollinearity had to be taken into account
} 

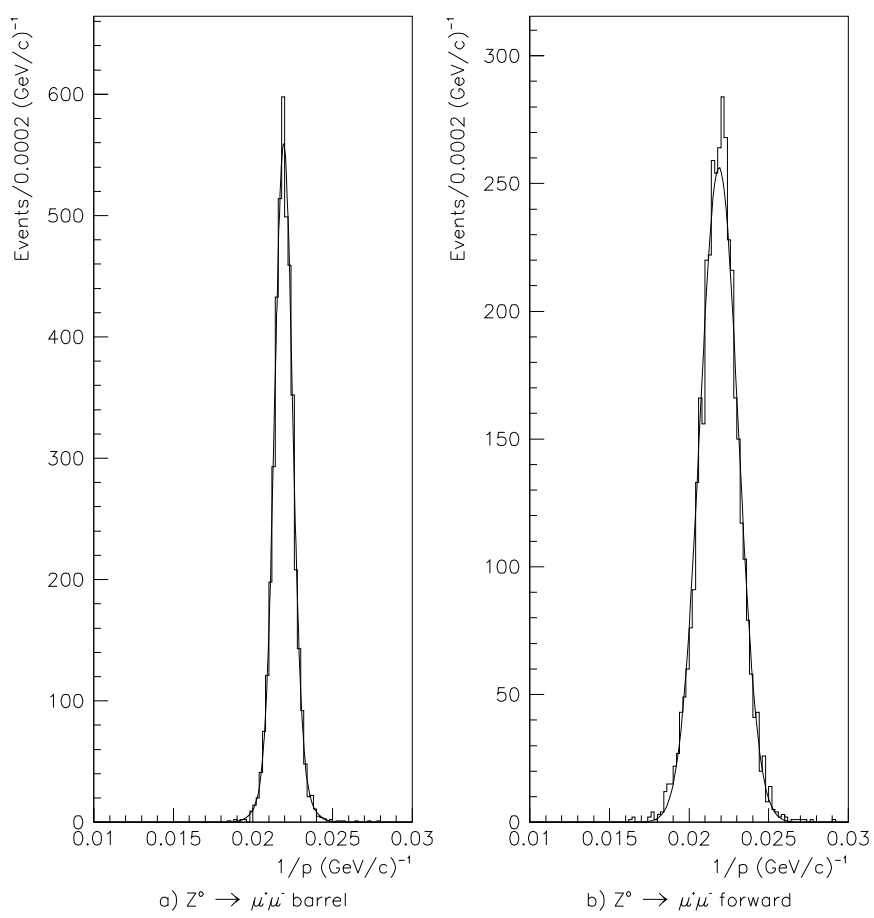

Figure 8: Inverse momentum distributions for collinear muons from $\mathrm{Z} \rightarrow \mu^{+} \mu^{-}$decays: (a) tracks containing hits from VD, ID, TPC and OD, (b) tracks containing hits from VD and FCB at least.

\begin{tabular}{|r|l|c|}
\hline$\theta\left(^{\circ}\right)$ & Detectors & $\sigma(1 / p)(\mathrm{GeV} / c)^{-1}$ \\
\hline$\geq 42$ & VD+ID+TPC+OD & $0.6 \times 10^{-3}$ \\
$\geq 42$ & ID+TPC+OD & $1.1 \times 10^{-3}$ \\
$\geq 42$ & VD+ID+TPC & $1.7 \times 10^{-3}$ \\
$\leq 36$ & VD + FCB included & $1.3 \times 10^{-3}$ \\
$25-30$ & FCB included & $1.5 \times 10^{-3}$ \\
$<25$ & FCB included & $2.7 \times 10^{-3}$ \\
\hline
\end{tabular}

Table 4: Momentum measurement precision for $45.6 \mathrm{GeV} / c$ muons. 

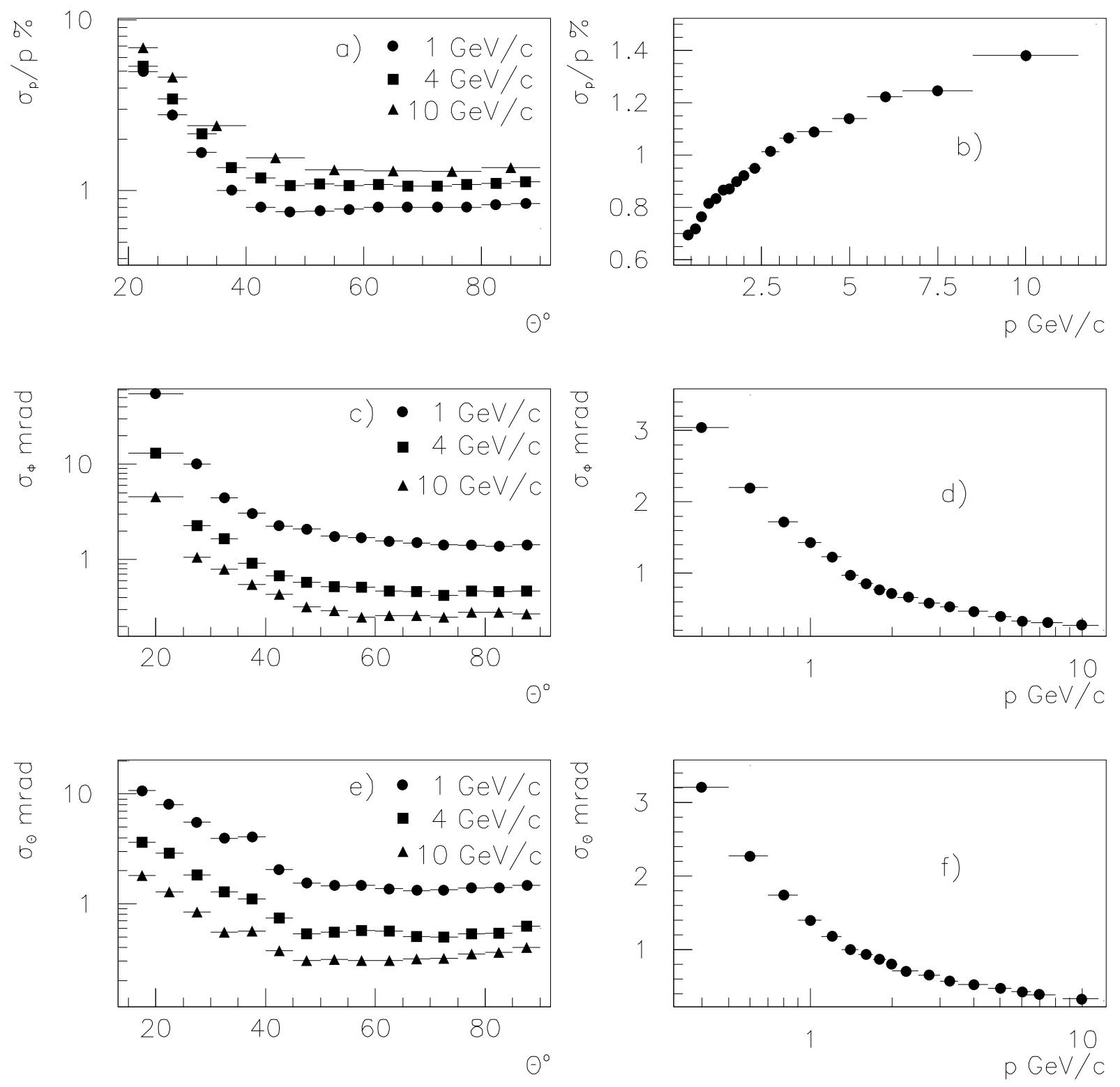

Figure 9: Track parameter precisions estimated by comparing simulated and reconstructed parameters: (a) momentum precision as a function of the polar angle $\theta$, (b) momentum precision as a function of the momentum for barrel tracks, (c) azimuthal angle precision as a function of $\theta$, (d) azimuthal angle precision as a function of the momentum for barrel tracks, (e) polar angle precision as a function of $\theta$, (f) polar angle precision as a function of the momentum for barrel tracks. 


\subsection{Charmed Particle Mass Reconstruction}

The $\mathrm{K}^{-} \pi^{+}$invariant mass distribution in the decay chain $\mathrm{D}^{*+} \rightarrow \mathrm{D}^{0} \pi^{+} \rightarrow \mathrm{K}^{-} \pi^{+} \pi^{+}$is an illustration of the mass resolution achieved by the tracking system. The width of the $\mathrm{D}^{0}$ peak is $23 \pm 1 \mathrm{MeV} / \mathrm{c}^{2}$ and the fitted $\mathrm{D}^{0}$ mass is $1863 \pm 1 \mathrm{MeV} / \mathrm{c}^{2}$. Masses and mass resolutions obtained from the study of some exclusive D meson decay channels are given in Table 5.

\begin{tabular}{|c|l|c|c|}
\hline Channel & & $0.15<E_{\mathrm{D}} / E_{\text {beam }}<0.55$ & $E_{\mathrm{D}} / E_{\text {beam }}>0.55$ \\
\hline $\mathrm{D}^{*+} \rightarrow \mathrm{D}^{0} \pi^{+}$ & $\sigma\left(M_{\mathrm{K} \pi \pi}-M_{\mathrm{K} \pi}\right)$ & $1.0 \pm 0.1 \mathrm{MeV} / c^{2}$ \\
$\mathrm{D}^{0} \rightarrow \mathrm{K}^{-} \pi^{+}$ & $M_{\mathrm{D}^{0}}$ & $1862 \pm 1 \mathrm{MeV} / c^{2}$ & $1866 \pm 2 \mathrm{MeV} / c^{2}$ \\
& $\sigma\left(M_{\mathrm{D}^{0}}\right)$ & $22 \pm 1 \mathrm{MeV} / c^{2}$ & $30 \pm 2 \mathrm{MeV} / c^{2}$ \\
\hline $\mathrm{D}^{0} \rightarrow \mathrm{K}^{-} \pi^{+}$ & $M_{\mathrm{D}^{0}}$ & $1864 \pm 1 \mathrm{MeV} / c^{2}$ & $1867 \pm 2 \mathrm{MeV} / c^{2}$ \\
& $\sigma\left(M_{\mathrm{D}^{0}}\right)$ & $1869 \pm 1 \mathrm{MeV} / c^{2}$ & $25 \pm 2 \mathrm{MeV} / c^{2}$ \\
\hline $\mathrm{D}^{+} \rightarrow \mathrm{K}^{-} \pi^{+} \pi^{+}$ & $M_{\mathrm{D}^{+}}$ & $15 \pm 1 \mathrm{MeV} / c^{2}$ & $1868 \pm 1 \mathrm{MeV} / c^{2}$ \\
& $\sigma\left(M_{\mathrm{D}^{+}}\right)$ & $21 \pm 2 \mathrm{MeV} / c^{2}$ \\
\hline
\end{tabular}

Table 5: Masses and mass resolutions for D mesons; the decay $\mathrm{D}^{0} \rightarrow \mathrm{K}^{-} \pi^{+}$appears twice (tagged via $\mathrm{D}^{*}$ and inclusively).

\section{$8 V^{0}$ Reconstruction}

Candidate $V^{0}$ decays in hadronic events are found by considering all pairs of oppositely charged particles. The vertex defined by each pair is determined by minimising the $\chi^{2}$ obtained from the distances from the vertex to the extrapolated tracks (considered as ellipsoids in the $5 D$ space of the track parameters).

The "loose" $V^{0}$ sample is defined by the following criteria:

- the angle $\Delta \phi$ in the $x y$ plane between the $V^{0}$ momentum and the line joining the primary to the secondary vertex is less than $0.1 \mathrm{rad}$;

- the radial separation $R$ of the primary and secondary vertex in the $x y$ plane is greater than twice the error on the fitted distance;

- the probability of the $\chi^{2}$ fit to the secondary vertex is larger than 0.001 ;

- the transverse momentum of each particle of the $V^{0}$ with respect to the line of flight is greater than $0.02 \mathrm{GeV} / c$ and the invariant mass in the $e^{+} e^{-}$hypothesis is more than $0.16 \mathrm{GeV} / \mathrm{c}^{2}$;

- when the reconstructed decay point of the $V^{0}$ is beyond the VD radius, there is no signal in the VD consistent with association to the decay vertex.

A $V^{0}$ candidate is in the "standard" sample if it also satisfies the requirements:

- $\Delta \phi<\left(0.01+0.02 / p_{t}\right) \mathrm{rad}$, where $p_{t}$ is the transverse momentum of the $V^{0}$ candidate relative to the beam axis, in $\mathrm{GeV} / c$;

- $R>4 \sigma$;

- probability of the $\chi^{2}$ fit larger than 0.01 .

The $\pi^{+} \pi^{-}$and $\mathrm{p} \pi^{-}\left(\overline{\mathrm{p}} \pi^{+}\right)$invariant masses are calculated (attributing the proton mass to the higher momentum particle) and mass constrained fits are also performed for the $\mathrm{K}^{0}$ and $\Lambda(\bar{\Lambda})$ hypotheses. When a pair is consistent within three standard deviations with both $\mathrm{K}^{0}$ and $\Lambda(\bar{\Lambda})$ hypotheses, the one with the smaller mass pull (the absolute 
mass shift from the nominal mass divided by its error) is selected. Finally, "tight" $\mathrm{K}^{0}$ or $\Lambda(\bar{\Lambda})$ flag is set if respectively:

- $0.35<m_{\pi \pi}<0.65 \mathrm{GeV} / c^{2}$;

- $0.02<$ probability to have decayed within the fitted distance $<0.95$,

or:

- $m_{\pi p}<1.3 \mathrm{GeV} / c^{2}$

- $0.02<$ probability to have decayed within the fitted distance $<0.95$,

and the invariant mass and the nominal mass are equal within two standard deviations.
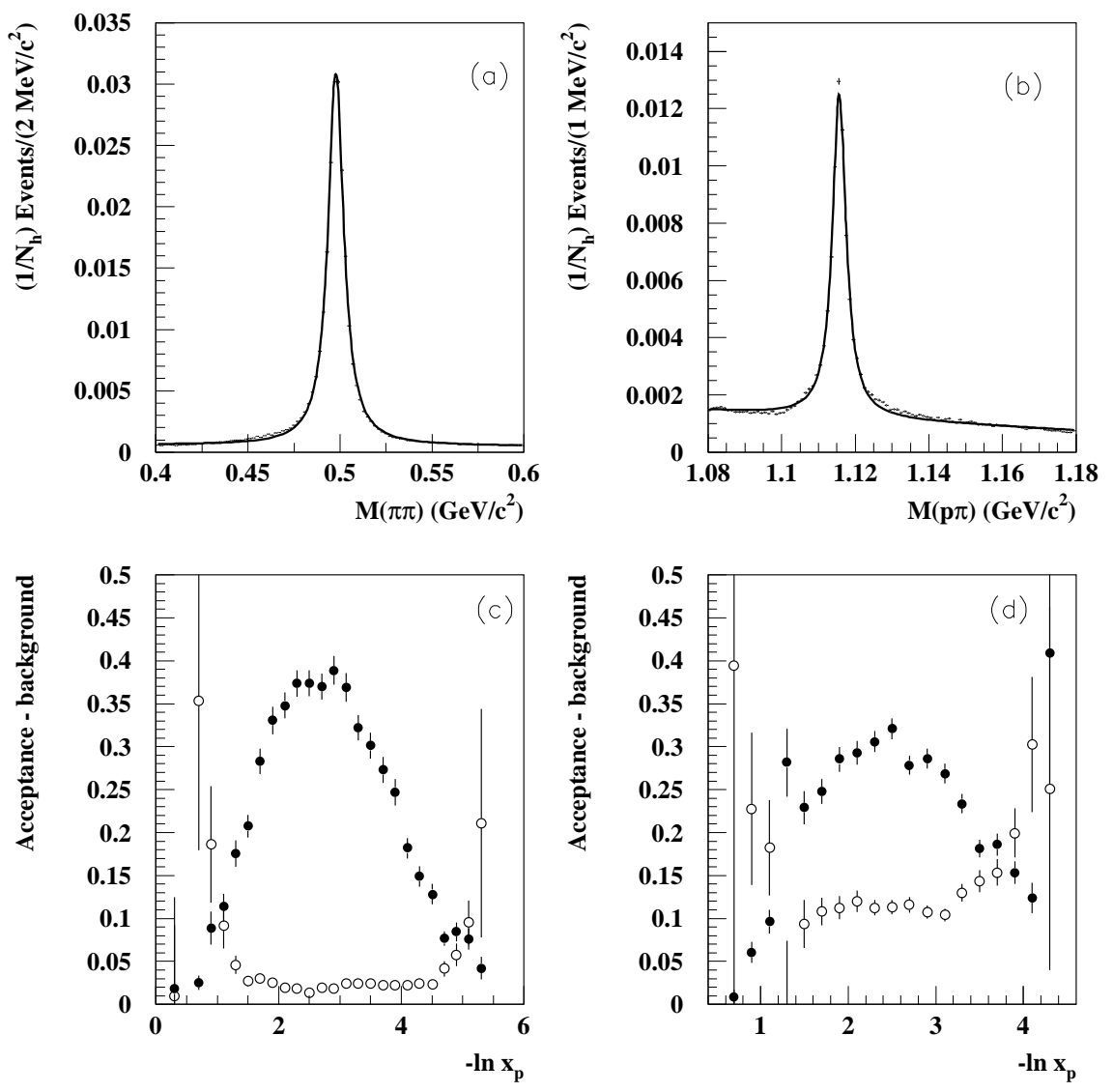

Figure 10: Invariant mass distribution for the tight (a) $\mathrm{K}^{0}$ and (b) $\Lambda$ samples, normalized to the total number of hadronic events; the line shows a fit to a Breit-Wigner shape for the mass plus a linear background. Efficiency (closed circles) and background fraction (open circles) as a function of $-\ln x_{p}=$ $-\ln p / p_{\text {beam }}$ for tight (c) $\mathrm{K}^{0}$ and (d) $\Lambda$ samples.

The reconstructed invariant mass distributions for a sample of tight $\mathrm{K}^{0}$ and $\Lambda$ from the 1994 data sample are shown in Figures 10(a) and 10(b) respectively. The mean mass resolution, defined as the FWHM of the fitted distributions, is $4.3 \mathrm{MeV} / c^{2}$ for the $\mathrm{K}^{0}$ and $1.8 \mathrm{MeV} / \mathrm{c}^{2}$ for the $\Lambda$. The efficiency depends strongly on the $V^{0}$ momentum: Figures $10(\mathrm{c})$ and $10(\mathrm{~d})$ show the efficiency and contamination as a function of $\xi=-\ln \left(\mathrm{p} / \mathrm{p}_{\text {beam }}\right)$. for the same data samples. The efficiency for $\mathrm{K}^{0} \rightarrow \pi^{+} \pi^{-}$in the tight selection averaged 
over the momentum spectrum is about $36 \%$ with a contamination of $3 \%$. The average efficiency for $\Lambda \rightarrow p \pi$ is $30 \%$ with a contamination of about $10 \%$.

\section{$9 \quad b$ Tagging}

Tagging events containing $b$ quarks is based on reconstructing as precisely as possible a) the position in space of the primary $Z$ decay and $b$ ) the impact parameters of the outgoing tracks with respect to that vertex, and then c) applying an algorithm to use this information in an optimal way. The following subsections describe these three steps.

\subsection{Primary Vertex Reconstruction}

The primary vertex is reconstructed for every hadronic event using the beamspot position as a constraint. The beamspot is defined as the interaction region of the electron and positron beams. To follow variations during a LEP fill, its position is determined for every cartridge written by the DAS corresponding to about 200 hadronic events. A common vertex is fitted using tracks with at least 2 hits in the VD, and the horizontal $(x)$ and vertical $(y)$ position of the beam and its horizontal width are determined. The $x$ and $y$ positions are found with typical uncertainties of about $9 \mu \mathrm{m}$ and $4 \mu \mathrm{m}$ respectively. The width along the $x$ coordinate varies with time but a typical value is 100 to $120 \mu \mathrm{m}$ with an error of $7 \mu \mathrm{m}$.

The beamspot is small, which improves the accuracy of the event by event primary vertex fit and therefore the efficiency for tagging $b$ quark events. Tracks with wrong associations to hits in the vertex detector, from secondary decays of long lived particles or from interactions in the detector material, may spoil the reconstruction of the vertex. To minimize the presence of these tracks, only tracks with 2 or 3 VD hits are used in the primary vertex fit. Moreover for each track the quantity $\Sigma_{\text {hits }}=\Sigma\left(d_{i}^{2} / \sigma_{V D}^{2}\right)$ is computed, where the sum is over all the $N_{V D}$ hits in the vertex detector associated to it, $d_{i}$ is the distance of closest approach of the track to the VD hit and $\sigma_{V D}$ is the accuracy of the VD hits; it is required that $\Sigma_{\text {hits }} / N_{V D} \leq 4$. In addition, tracks should be close to the beamspot position in terms of $\delta / \sigma$, where $\delta$ is the distance from the track to the beamspot and $\sigma$ is the corresponding error, including the track error and the beamspot size. Specifically, the confidence level computed for a given $\delta / \sigma$ is required to be greater than 0.05 . The primary vertex position is obtained by minimizing the $\chi^{2}$ function given by [44]:

$$
\chi^{2}\left(V_{i}\right)=\sum_{a} \frac{\bar{d}_{a}^{2}}{\sigma_{a}^{2}}+\sum_{i=x, y, z} \frac{\left(b_{i}-V_{i}\right)^{2}}{\left(\sigma_{i}^{b}\right)^{2}},
$$

where $\bar{d}_{a}$ is the distance of the track $a$ to the fitted vertex, $\sigma_{a}$ is the corresponding error, $V_{i}$ is the coordinate of the primary vertex and $b_{i}, \sigma_{i}^{b}$ are the beamspot position and size. The fit is done iteratively, excluding after every iteration the track giving the largest difference $\chi^{2}\left(N_{t r}\right)-\chi^{2}\left(N_{t r}-1\right)$ if it exceeds a cutoff value $\Delta_{\max }$. Since the beamspot is used as a starting reference point, in principle all the tracks can be rejected from the fit. For these events the beamspot centre is taken as the primary vertex and the covariance matrix corresponds to the beamspot size. The fraction of such events is around $1 \%$.

The main advantage of this method of fitting is the quadratic dependence of $\chi^{2}$ on the fitted values (see Equation 3). It gives the possibility of analytical and straightforward determination of the vertex position $V_{i}$. The simple form of Equation 3 helps also in the calculation of the impact parameter errors (see below). Figure 11 shows the difference 

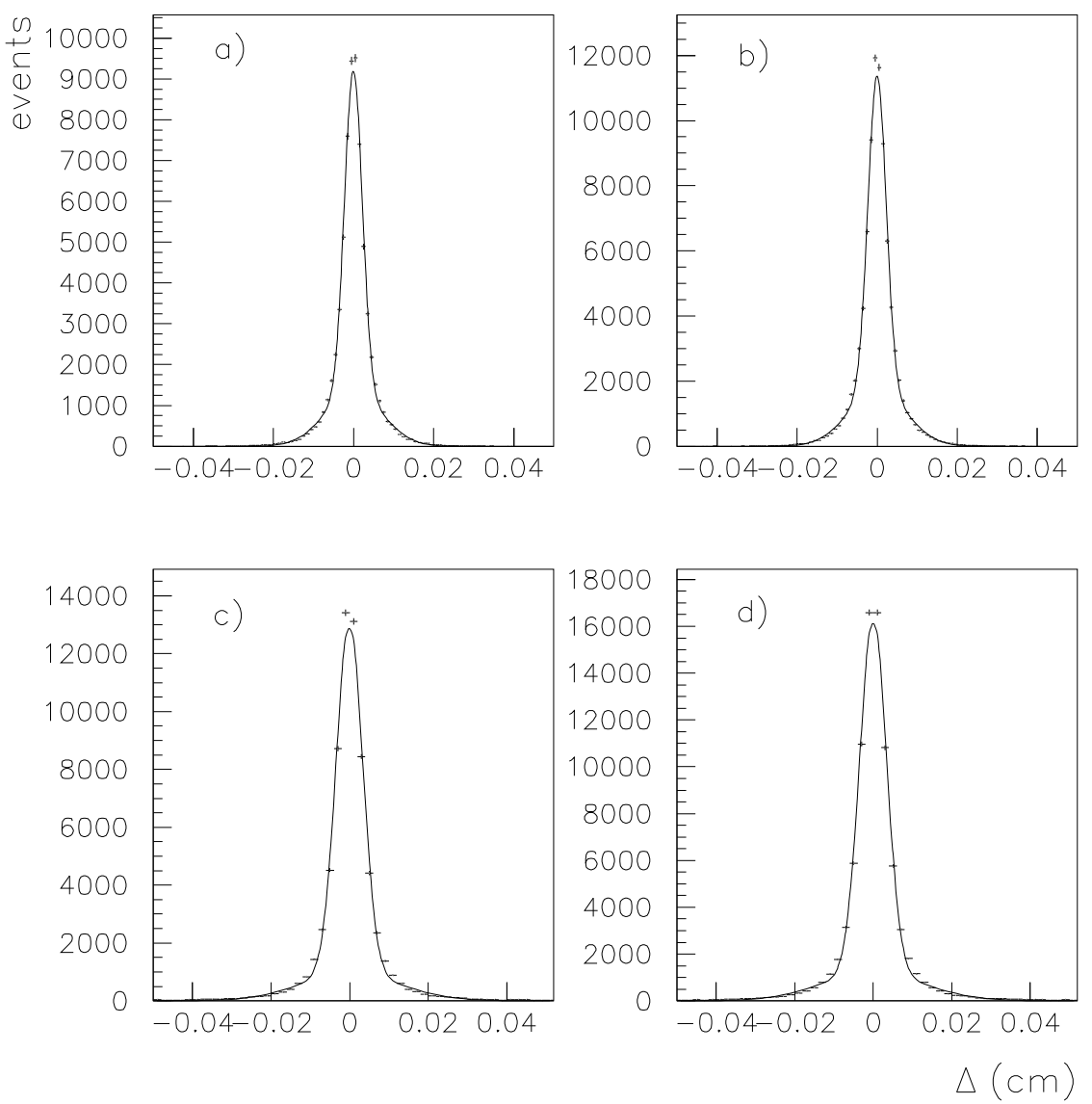

Figure 11: Difference between the reconstructed and generated vertex position in a simulated event sample for (a) $x$-coordinate for light quarks, (c) $x$-coordinate for $b$ quarks, (b) $z$-coordinate for light quarks and (d) $z$-coordinate for $b$ quarks. The full lines show fits to the data with a sum of two Gaussians. 
between the reconstructed and generated vertex position in a simulated event sample in the $x$ direction (a) for light quark events and (c) for $b$ quark events, and in the $z$ direction (b) for light quark events and (d) for $b$ quark events. The widths of the distributions are about $22 \mu \mathrm{m}$ in the $x$ and $z$ directions for light quark events and $35 \mu \mathrm{m}$ for $b$ quark events. The distributions for $b$ quark events show pronounced non-Gaussian tails due to the inclusion of tracks coming from secondary vertices.

Before 1994 the VD did not provide measurements of the $z$ coordinate. In this situation the selection of the tracks coming from the primary vertex was less precise, so the resolutions in $x$ for light quarks and $b$ quarks were about $50 \%$ larger and the non-Gaussian tails of the distributions were considerably more pronounced.

\subsection{Impact Parameter Precision}

The impact parameter is defined as the distance of closest approach of a charged particle to the reconstructed primary vertex. The impact parameters in the $R \phi$ and $R z$ planes are evaluated separately.

The sign of the impact parameter is defined with respect to the jet direction. It is positive if the vector joining the primary vertex to the point of closest approach of the track is less than $90^{\circ}$ from the direction of the jet to which the track belongs. The sign is computed in 2 dimensions when only $R \phi$ measurements from the VD are available, in 3 dimensions when the $z$ information is available. $R \phi$ and $R z$ impact parameters are given the same sign for a given track. The impact parameter error is due to the track extrapolation error on the point of closest approach and the error on the primary vertex and consequently depends on the beamspot size.

The measurement contribution to the track extrapolation error at the interaction point can be estimated from the apparent distance between the tracks from $\mathrm{Z} \rightarrow \mu^{+} \mu^{-}$decays, where multiple scattering and vertex contributions are negligible. Figure 12, upper, shows this distance projected onto the $R \phi$ plane when the muon energies are constrained to the beam energy. The $28 \mu \mathrm{m}$ width of this distribution indicates a track extrapolation measurement error of $\sigma_{I P R \phi}=20 \mu \mathrm{m}$. In the $R z$ plane, the dimuon miss distance precision varies as a function of $\theta$ (see Figure 12, lower). The extrapolation error is $\sigma_{I P z}=34 \mu \mathrm{m}$ for tracks at normal incidence.

Track extrapolation precisions at lower momenta can be estimated using a sample enriched in light quark events, selected by a cut on the probability $P_{E}$ (see next subsection) computed from tracks having positive impact parameter, and then using tracks with negative impact parameters to avoid bias. After subtracting the vertex position uncertainty in quadrature, the extrapolation errors in the $R \phi$ and $R z$ planes are parametrised as:

$$
\sigma_{I P_{R \phi}}{ }^{2}=\left(\frac{\alpha_{M S}}{p \sin ^{3 / 2} \theta}\right)^{2}+\left(\sigma_{0, R \phi}\right)^{2} \quad \sigma_{I P_{Z}}{ }^{2}=\left(\frac{\alpha_{M S}^{\prime}}{p \sin ^{5 / 2} \theta}\right)^{2}+\left(\sigma_{0, R z}\right)^{2}
$$

where $\alpha_{M S}\left(\alpha_{M S}^{\prime}\right)$ is a multiple scattering coefficient (in $\mu \mathrm{m} \cdot \mathrm{GeV} / c$ ) and $p$ is the track momentum. In both expressions, the first term is the multiple scattering contribution and the second is due to measurement error. The upper curve in Figure 13, upper, shows the extrapolation error in the $R \phi$ plane as a function of $p \sin ^{3 / 2} \theta$. To obtain these values, the vertex position uncertainty, shown by the lower curve, has been subtracted from the measured impact parameter error. Parametrising the extrapolation uncertainty as above gives $\alpha_{M S}=65 \mu \mathrm{m} \cdot \mathrm{GeV} / c, \sigma_{0, R \phi}=20 \mu \mathrm{m}$.

The extrapolation error in the $R z$ plane depends strongly on the polar angle of the track. Two effects contribute to the degradation of the extrapolation precision for non- 

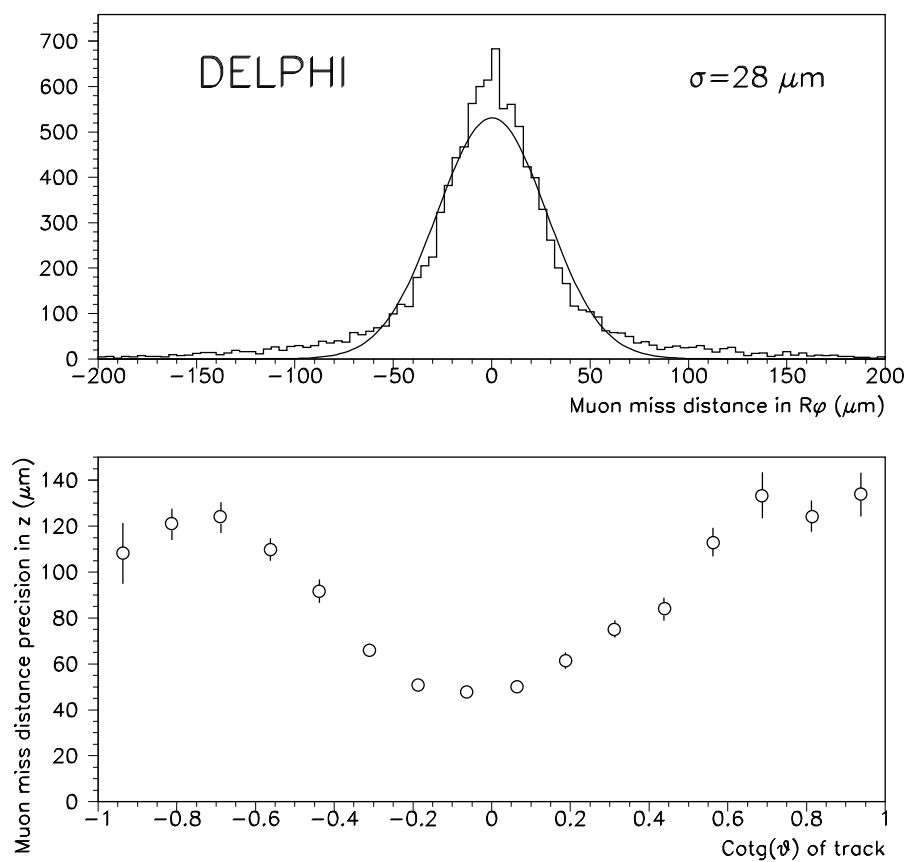

Figure 12: Upper: miss distance between the two muons in the $R \phi$ plane for $\mathrm{Z} \rightarrow \mu^{+} \mu^{-}$events. The non Gaussian tails are due to variations in the VD hit precision. Lower: miss distance precision in the $R z$ plane for $\mathrm{Z} \rightarrow \mu^{+} \mu^{-}$events, as a function of the polar angle. 

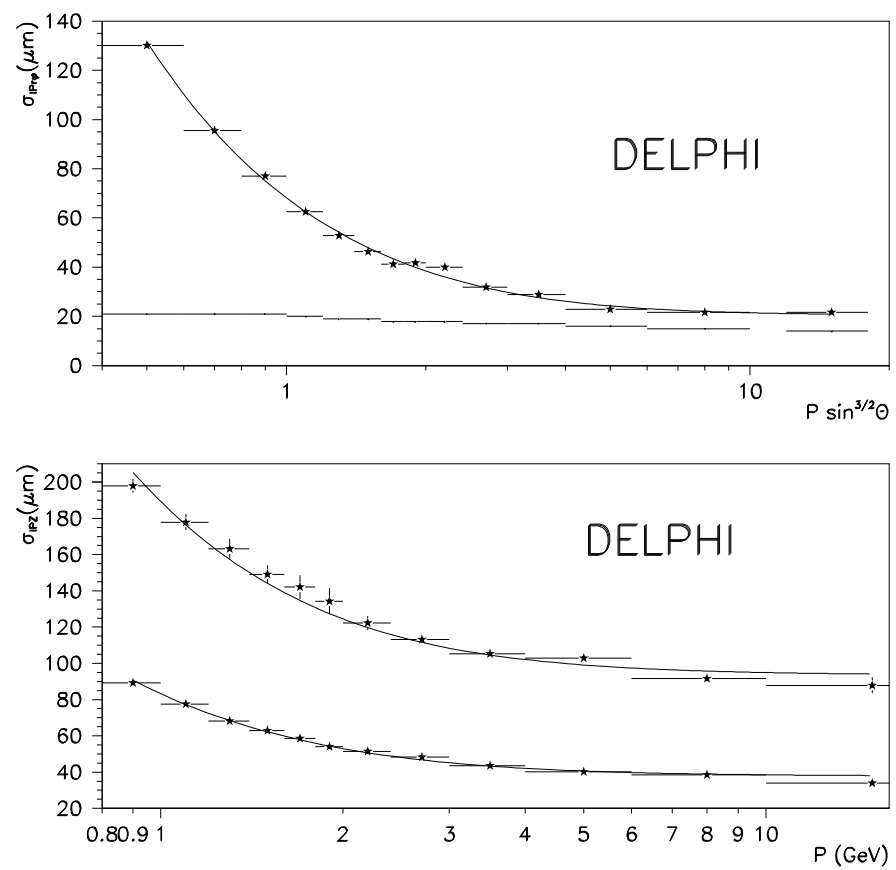

Figure 13: Upper: Mean track extrapolation uncertainty in $R \phi$ measured as a function of $p \sin ^{3 / 2} \theta(p$ in $\mathrm{GeV} / c$ ). The values are determined by subtracting quadratically the vertex position uncertainty, shown by the bottom curve, from the measured impact parameter uncertainty. The full line depicts $\left(65 / p \sin ^{3 / 2} \theta \oplus 20\right) \mu \mathrm{m}$. Lower: Mean track extrapolation uncertainty in the $R z$ plane, measured similarly as a function of the particle momentum. The two curves correspond to tracks with $80^{\circ}<\theta<90^{\circ}$ (bottom) and $45^{\circ}<\theta<55^{\circ}$ (top). The full lines depict $(71 / p \oplus 39) \mu \mathrm{m}$ and $(151 / p \oplus 96) \mu \mathrm{m}$ respectively. 
perpendicular tracks. The first is the varying point precision in $z$ which affects the measurement error; the second is the larger path in the material which increases the multiple scattering uncertainty. Figure 13, lower, shows the extrapolation error in $R z$ as a function of momentum for $45^{\circ}<\theta<55^{\circ}$, upper curve, and $80^{\circ}<\theta<90^{\circ}$, lower curve. The measurement error values are $96 \mu \mathrm{m}$ and $39 \mu \mathrm{m}$ respectively, matching well the result obtained from the dimuon miss distance at the same angles. The multiple scattering coefficient $\alpha_{M S}^{\prime}$ is $71 \mu \mathrm{m} \cdot \mathrm{GeV} / c$.

The effect of adding the $z$ information can be seen by comparing the impact parameter resolution in the $R z$ plane for nearly perpendicular tracks $\left(70^{\circ}<\theta<110^{\circ}\right)$ above $6 \mathrm{GeV} / c$, without and with $z$ hits. Adding the $z$ hits gives nearly a factor 20 improvement in the $R z$ impact parameter precision, from $884 \mu \mathrm{m}$ to $47 \mu \mathrm{m}$.
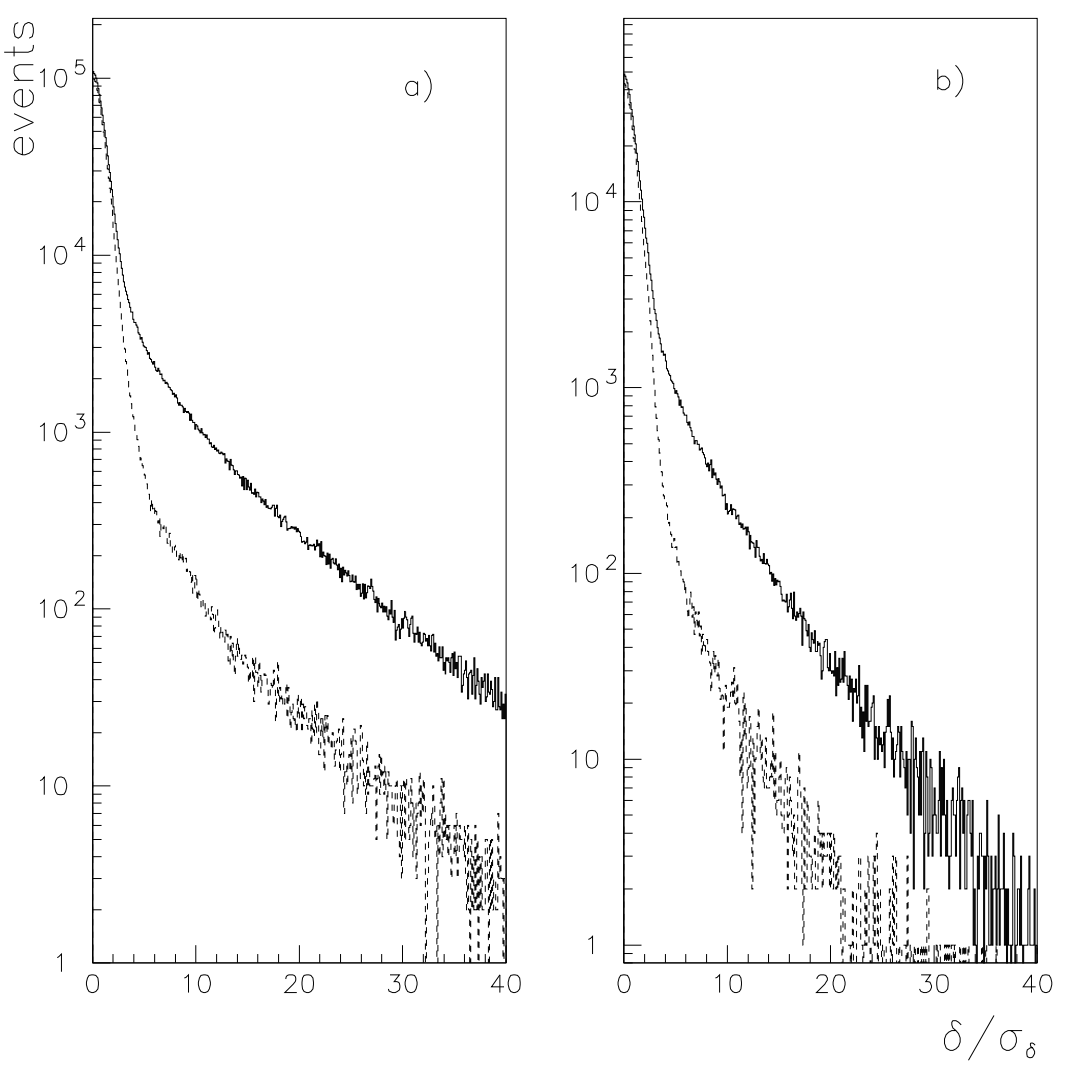

Figure 14: Distribution of the absolute value of the significance in $R \phi$ (a) and $R z$ (b) for tracks from real data measured in the VD with negative (dashed line) and positive (solid line) impact parameters.

The significance is defined as the ratio of the impact parameter value to its error. The positive and negative $R \phi$ and $R z$ significance distributions are shown in Figures 14(a) and 14(b) respectively. In the ideal case the negative significance distribution should have a Gaussian shape, but, as can be seen from Figure 14, there is a long non-Gaussian tail. This is due largely to tracks measured wrongly by the tracking system and partly to particles from secondary decays and interactions. The Gaussian part of the significance distribution is well understood and may be measured directly from the data, while the 
non-Gaussian tail depends significantly on the criteria which are used for the selection of tracks and events.

\subsection{The $b$ Tagging Algorithm}

Hadrons containing $b$ quarks have long lifetimes (typically $1.6 \mathrm{ps}$ ) and large masses, so they have many decay products with large impact parameters and this can be used to separate events in which $b$ quarks are produced from other hadronic events. With the definition of the impact parameter and of its sign given above, it follows that the tracks from the decays of $B$ hadrons should have positive impact parameters, whereas non-zero impact parameters arising from inaccurate track reconstruction should be equally likely to be positive or negative. Therefore using tracks with positive impact parameters increases the tagging performance.

For tagging $B$ hadrons, the probability method described in [44] is used. This gives the possibility of building one tagging variable from all the impact parameter values observed in the event. A very pure sample with a high tagging efficiency can be obtained for events with $B$ hadrons.

For $b$ tagging, tracks are selected as for the vertex fit (Section 9.1). The negative significance distribution mainly reflects the detector resolution and is used to build the track probability function $P\left(S_{0}\right)$, which is the probability for a track from the primary interaction to have a significance with absolute value $S_{0}$ or greater.

Using the track probability function, the probability for each track in the event can be computed according to the value of the significance. The N-track probability is defined as:

$$
P_{N} \equiv \Pi \cdot \sum_{j=0}^{N-1}(-\ln \Pi)^{j} / j !, \text { where } \Pi \equiv \prod_{i=1}^{N} P\left(S_{i}\right) .
$$

This variable gives the probability for a group of $\mathrm{N}$ tracks with the observed values of significance all to come from the primary vertex. By construction, the distribution of $P_{N}$ should be flat for groups of tracks from the primary vertex, provided the significances of these tracks are uncorrelated, while for $b$ quarks it should have a sharp peak at 0 . When the $R z$ impact parameter is measured, the probability $P\left(S_{0}\right)_{z}$ is computed in the same way as for the $R \phi$ impact parameter. The N-track probability is then given by the combination of the $P_{r \phi}$ and $P_{z}$ probabilities.

The N-track probability is the only variable which is used in this approach for tagging $b$ hadrons. The event probability, $P_{E}$, is the probability computed using all tracks of the event. Similarly, the hemisphere or jet probabilities, $P_{H}$ or $P_{J}$, are the probabilities computed from the tracks belonging to a given hemisphere or jet.

Figure 15 shows the efficiency and purity of the tagged sample for different values of the cut on the event probability (upper) and on the hemisphere probability (lower) for the 3-coordinates VD (full line) and the 2-coordinates VD (dashed line). The curves were calculated for a sample of simulated hadronic events selected within the acceptance of the vertex detector $\left(\left|\cos \left(\theta_{\text {thrust }}\right)\right|<0.75\right)$. This selection corresponds to an efficiency of $69 \%$. It can be seen from the figure that the possibility of measuring both $R \phi$ and $R z$ increases the efficiency for a given purity. 

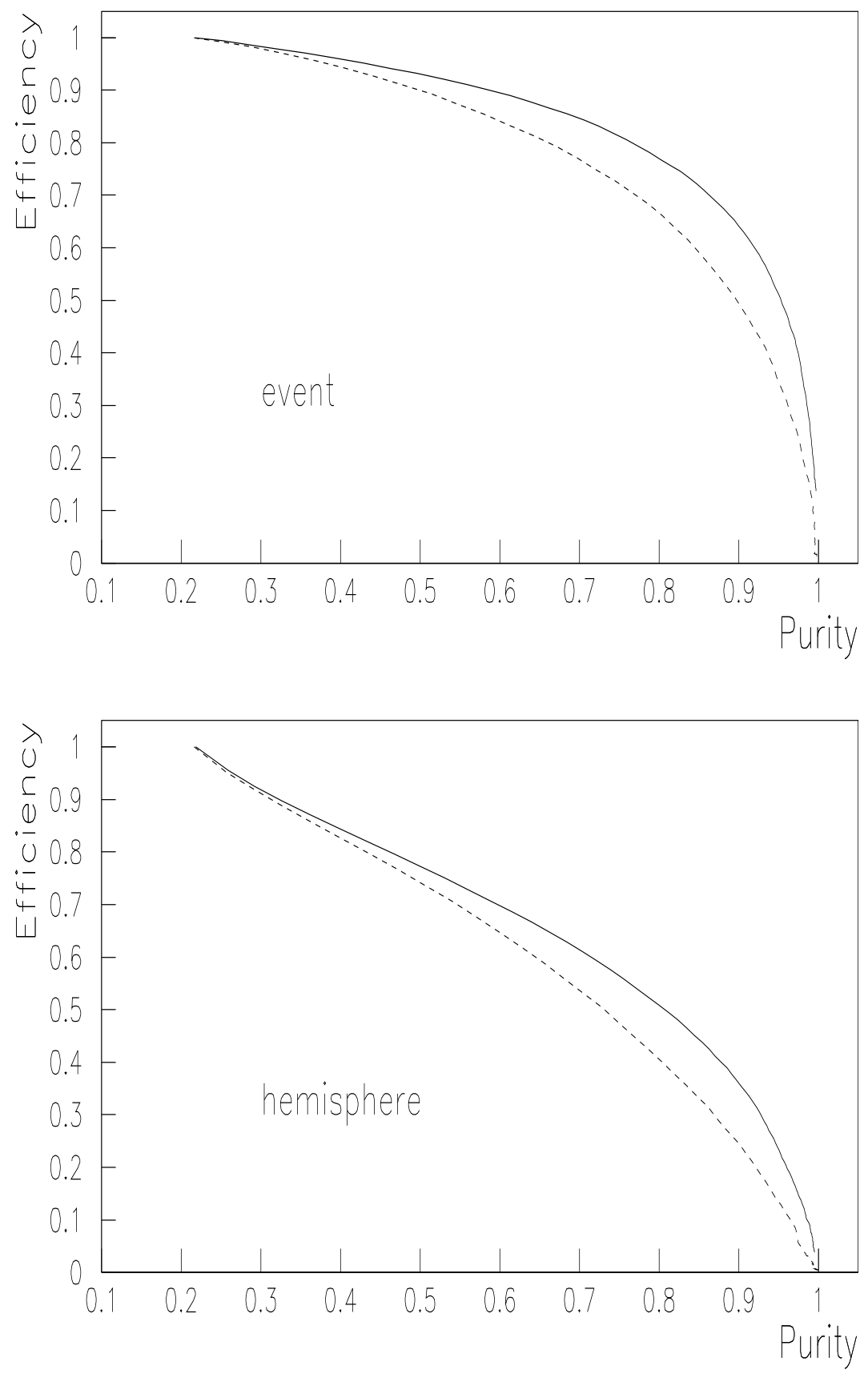

Figure 15: $b$-tagging efficiencies as a function of the purity of the tagged sample for different values of the cut on the event probability (upper) and on the hemisphere probability (lower) for the 3 -coordinates VD providing $R z$ information (full line) and the 2-coordinates VD that provided only $R \phi$ information (dashed line). 


\section{Charged Hadron Identification}

The identification of charged particles in DELPHI relies on the specific ionization energy loss per unit length $(d E / d X)$ in the TPC, on the RICH detectors, and on the electron and muon identification. In this section the performance of the charged hadron identification provided by the TPC and the RICH detectors is reviewed.

\subsection{Specific Ionization in the TPC}

The DELPHI TPC, in addition to providing three-dimensional track reconstruction, helps in charged particle identification by measuring the $d E / d X$. The sense wires of its proportional chambers provide up to 192 ionization measurements per track.

The signals collected by the sense wires are associated to the tracks reconstructed by the TPC pads. This association is done by comparing the arrival times of the pad and sense wire signals. Hits too close in time to be correctly separated are not used for the $d E / d X$ calculation. This requirement corresponds, for tracks orthogonal to the drift direction $(z)$, to a separation of at least $2 \mathrm{~cm}$. Sense wire signals with a width incompatible with a single track are also removed. On average $5 \%$ of the signals collected by the sense wires are below the electronic threshold. The fraction of the Landau distribution lost due to this effect is a function of the drift length and gap size (i.e. wire spacing as seen from the track). To reduce this dependence an effective threshold is applied which depends on these quantities. This contributes to a systematic loss of the lowest $\sim 8 \%$ of measurements. The highest $20 \%$ of signals are removed to reduce the influence of the Landau tail (that can be due to abnormal energy loss or to $\delta$ rays). After this cut, only $2 \%$ of the signals produced by electrons on the energy loss ("Fermi") plateau are still saturated. As well as reducing the number of saturated tracks, this last cut increases the relative height of the Fermi plateau, i.e. it increases by $10 \%$ the separation between particles on the Fermi plateau and minimum ionizing particles.

\begin{tabular}{|l|c|c|c|}
\hline & \multicolumn{2}{|c|}{ Tracks in Z $\rightarrow q \bar{q}$ events with } \\
& $0.2<p<1 \mathrm{GeV} / c$ & $p>1 \mathrm{GeV} / \mathrm{c}$ & $\begin{array}{c}\text { Leptons from Z } \rightarrow \ell \ell \\
p=45.6 \mathrm{GeV} / c\end{array}$ \\
\hline Barrel $(|\cos \theta|<0.7)$ & $82 \%$ & $61 \%$ & $97 \%$ \\
Endcap $(|\cos \theta|>0.7)$ & $78 \%$ & $67 \%$ & $97 \%$ \\
\hline
\end{tabular}

Table 6: Fractions of reconstructed tracks with at least $30 d E / d X$ measurements left in the truncated mean calculation.

To be used in the physics analysis, the $d E / d X$ value coming from the truncated mean is required to have at least 30 contributing measurements. The efficiencies obtained after all these requirements are given in Table 6 . The measured signals are corrected to take into account the remaining dependence on parameters like gap size or drift distance [45].

The dependence of $d E / d X$ on the momentum $p$ of the particle is measured from the data using various samples, and the final result can be seen in Figure 16 . The value of the Fermi plateau, normalized to the minimum ionizing particle, is found to be 1.52 units and for particles in jets the average precision estimated from the data (using pions from $\mathrm{K}_{S}^{0}$ decays) is about $7.4 \%$. Thus $\pi$ and $\mathrm{K}$ are separated at above the $1 \sigma$ level for momenta above $2 \mathrm{GeV} / c$. 


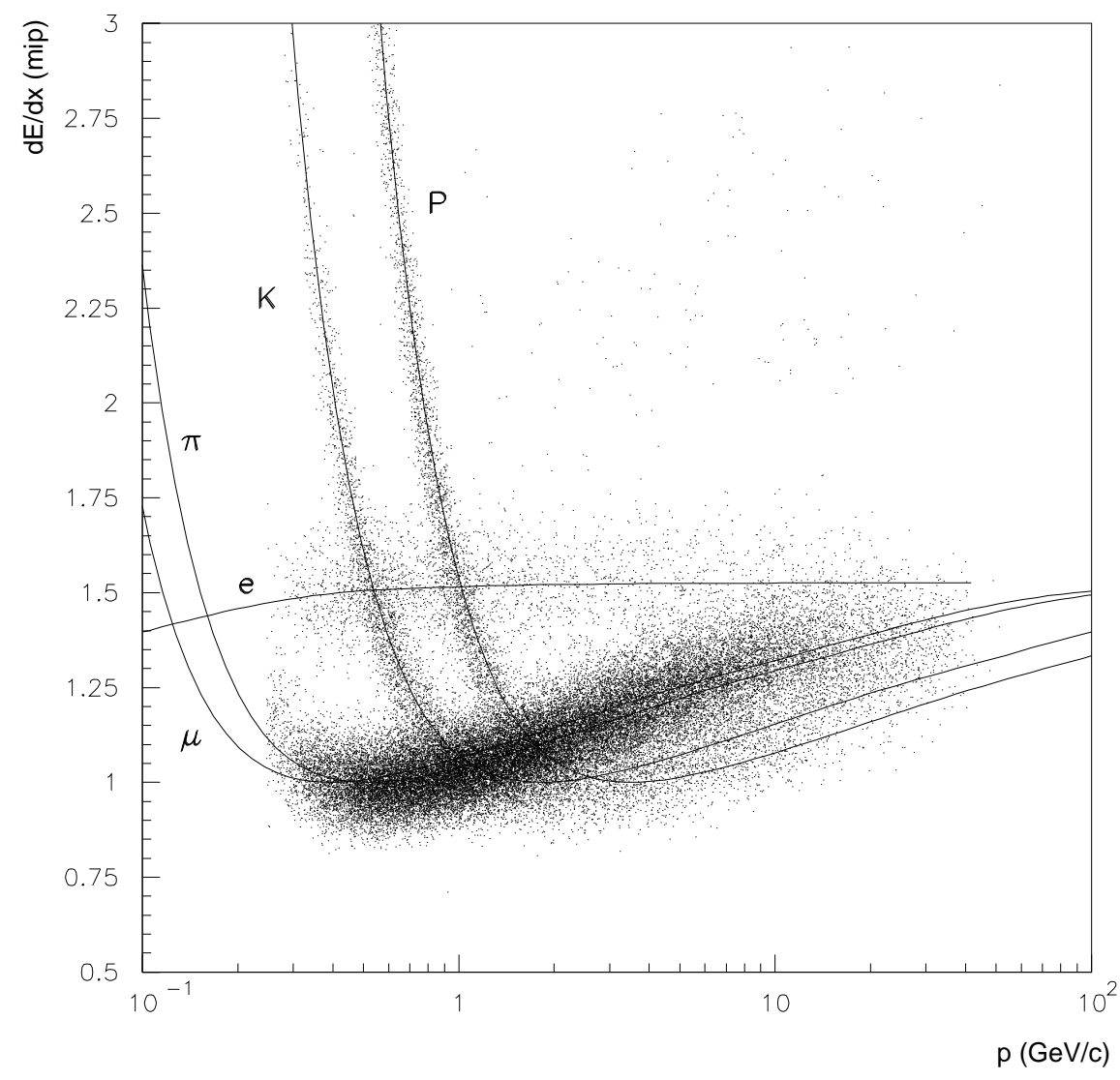

Figure 16: Specific energy loss $d E / d X$ in the TPC as a function of the momentum. 


\subsection{Ring Imaging Cherenkov Detectors}

Charged particles traversing a dielectric medium faster than the speed of light in that medium produce a cone of Cherenkov light. The emission angle $\theta_{c}$ depends on the mass $M$ and momentum $p$ via the relation $\cos \theta_{c}=1 / n \times \sqrt{1+M^{2} / p^{2}}$, where $n$ is the refractive index of the radiator medium. The number of photons emitted per unit length is proportional to $\sin ^{2} \theta_{c}$. The number of photons associated to a track and their Cherenkov angles are the input information used for identifying the particle mass. The fact that particles below the Cherenkov threshold do not emit light is also used ("veto identification").

The DELPHI RICH contains two radiators of different refractive indices. The liquid radiator is used for particle identification in the momentum range from 0.7 to $8 \mathrm{GeV} / \mathrm{c}$. The gas radiator is used from $2.5 \mathrm{GeV} / c$ to $25 \mathrm{GeV} / c$. It is kept at a temperature of $40^{\circ} \mathrm{C}$ and a pressure of 1033 mbar.

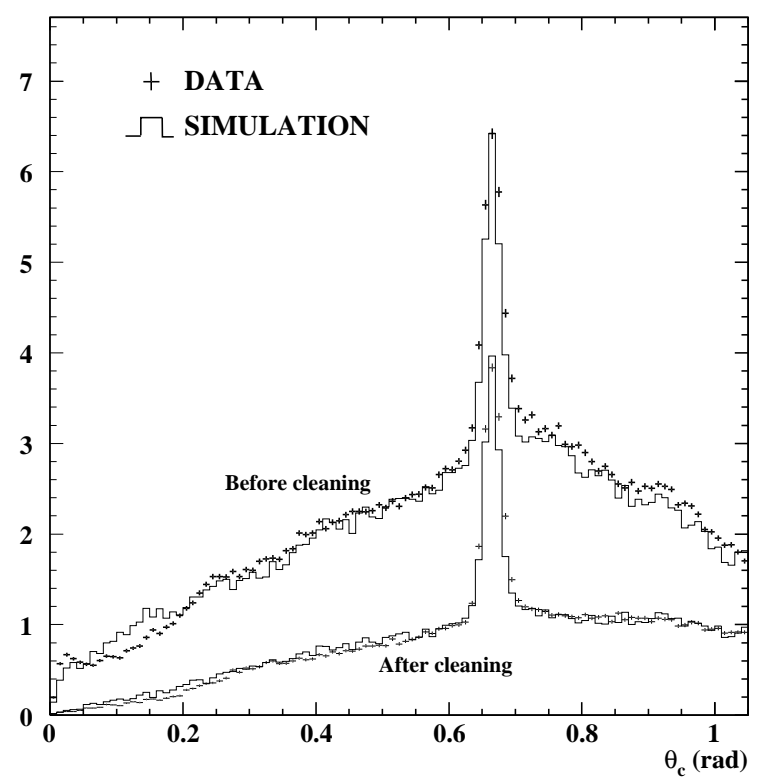

Figure 17: Cherenkov angles of individual photons in the barrel liquid radiator for charged particles with momenta above $6 \mathrm{GeV} / c$, before (upper line) and after (lower line) cleaning.

The full solid angle coverage is provided by two independent detectors, one in the endcap regions (Forward RICH), and one which covers polar angles between $40^{\circ}$ and $140^{\circ}$ (Barrel RICH) [46]. Perfluorocarbons were chosen as radiator media, both in the Forward (liquid $\mathrm{C}_{6} \mathrm{~F}_{14}$, gas $\mathrm{C}_{4} \mathrm{~F}_{10}$ ) and in the Barrel (liquid $\mathrm{C}_{6} \mathrm{~F}_{14}$, gas $\mathrm{C}_{5} \mathrm{~F}_{12}$ ). Photons in the range from 170 to $220 \mathrm{~nm}$ are focused onto photosensitive time projection chambers, 48 in number in the Barrel RICH and 24 in each arm of the Forward RICH. The three coordinates of the photon conversion point are determined by detecting the generated electron, referred to as the photoelectron. The emission angle of the photon with respect to the track (Cherenkov angle) is then reconstructed. An uncertainty is also computed, which depends on the photon conversion point and on the quality of the tracking information. In the first step of the data treatment, a cleaning algorithm removes detector and track related noise (several hundred photoelectrons, compared to a signal - see later - of the order of ten). In a hadronic event, the remaining background is due mainly to real photoelectrons, produced by nearby tracks (see Figure 17). 


\subsubsection{Monitoring and Alignment}

The identification power of the RICH depends on the accuracy of the Cherenkov angle measurement and on the number of photoelectrons detected. Stable operation of the different subsystems and monitoring of the relevant detector parameters is therefore very important.

The drift velocity and possible drift distortions are measured with a calibration system consisting of a matrix of fibres emitting UV light. In the Forward RICH, the Lorentz angle is determined by the same system. Longitudinal variations are also followed by comparing the ionizing tracks traversing the photon detector with the extrapolations from the tracking detectors. The final precision on the photon conversion point is measured to be within the design goal of $1 \mathrm{~mm}$.

Possible changes in the characteristics of the radiator media, which would induce variations of the refractive indices and the number of photoelectrons, are also monitored. The large number of events recorded allows a detailed study of the time evolution of the detector performance. The stability of the average number of photoelectrons per track in dimuon events was measured throughout the 1994 data taking period (where most of the statistics obtained with a fully operational detector were collected), and it was found to be excellent (the averages varied at most by \pm 0.5 photoelectrons).

Selected $\mathrm{Z} \rightarrow \mu^{+} \mu^{-}$events are used to align the different detector components, such as liquid radiator boxes, drift volumes and mirrors. A dedicated program minimises the difference between the observed and expected Cherenkov angles by varying the position of each component. Figure 18 shows the Cherenkov angle distributions obtained for dimuon events. The mean number of photoelectrons and the Cherenkov angle measurement precision depend on the position in the detector. The systematic uncertainties, in particular due to the track extrapolation, are different for each type of radiator. Table 7 shows average precisions both for single photoelectrons and per track. A detailed simulation program that takes into account all known detector effects has been tuned to reproduce the data.

\begin{tabular}{|l|c|c|c|c|}
\hline & B. liquid & B. gas & F. liquid & F. gas \\
\hline Number of photoelectrons per track & 14 & 8 & 7 & 8 \\
Cherenkov angle, mrad & 666 & 62.3 & 675 & 55.0 \\
Angular precision per photoelectron, mrad & 13.3 & 4.3 & 11.4 & 2.5 \\
Angular precision per track, mrad & 5.2 & 1.5 & 5.0 & 1.2 \\
\hline
\end{tabular}

Table 7: Numbers of photoelectrons and Cherenkov angles and precisions (in mrad) obtained in $\mathrm{Z} \rightarrow$ $\mu^{+} \mu^{-}$events, for the Barrel (B) and Forward (F) RICH.

\subsubsection{Identification Algorithms}

Several particle identification algorithms have been developed, in order to fulfil very different requirements. Some physics analyses need individual track tagging, while others measure statistically the content of a given sample, without associating tags to each track.

For track by track tagging, the observed signal is compared with that expected for known particle types, namely $e, \mu, \pi^{ \pm}, \mathrm{K}^{ \pm}$and $\mathrm{p}$, at the measured momentum. Depending on the analysis, the priority may be high rejection or high efficiency. The requirements also depends on the dominant source of combinatorial background, i.e. on whether only pion rejection is required or proton/kaon separation. 

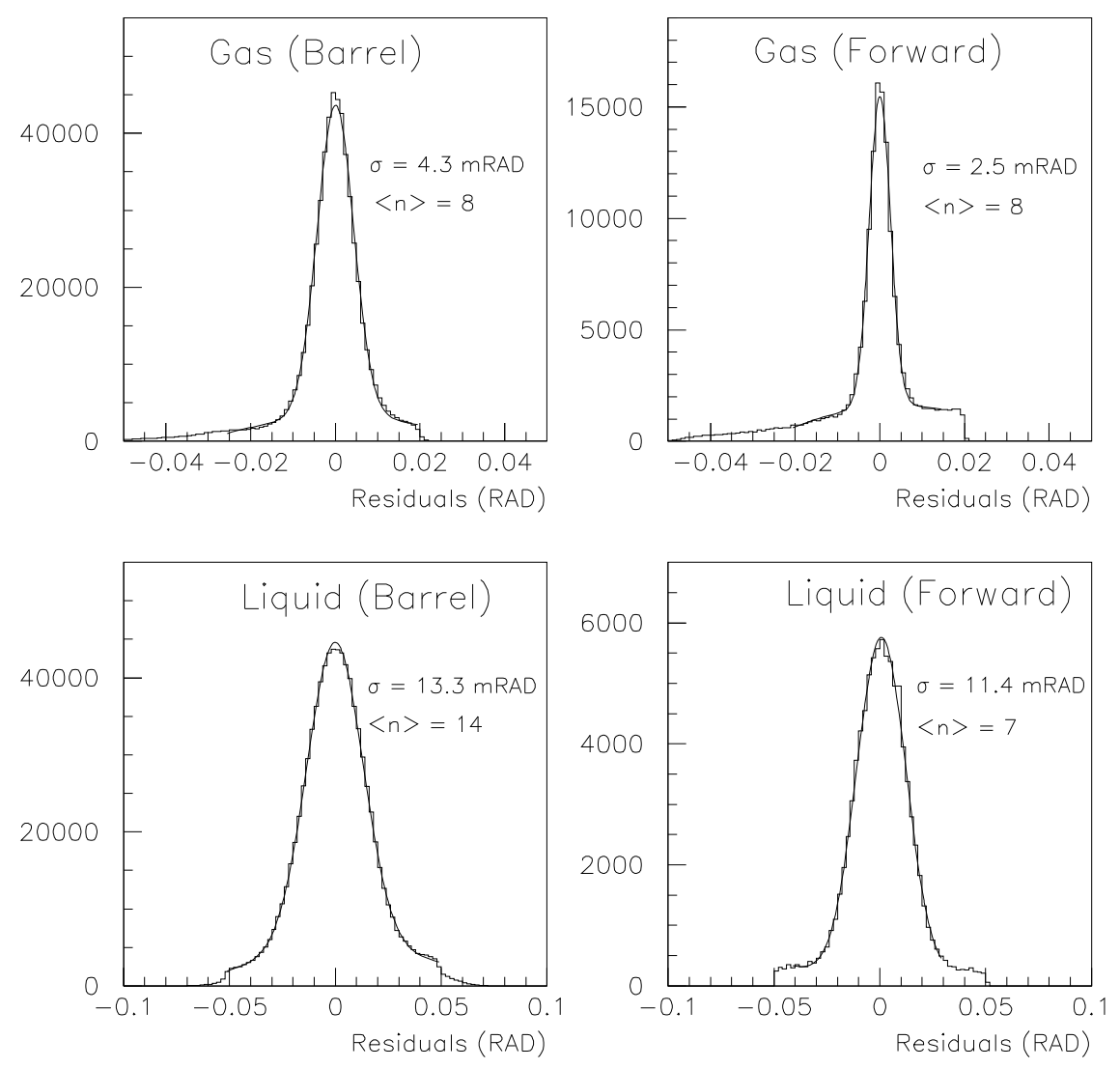

Figure 18: Distributions of the photoelectron Cherenkov angles, for $\mathrm{Z} \rightarrow \mu^{+} \mu^{-}$events. The average numbers of photoelectrons and the Cherenkov angle measurement precisions for single photons are given for both radiator types, gas and liquid, in both the Barrel and Forward RICH. 
For statistical analyses, one needs a continuous estimator of the observed Cherenkov angle, independent of any mass hypothesis, such that the number of particles of a given type can be determined.

In a hadronic event, the main difficulty is to deal with the background under the Cherenkov signal, whose shape and level is different for each track and a priori unknown. The algorithms developed so far follow two main approaches.

In the first approach (conventionally referred to as the "HADSIGN" approach), a flat background is fitted and no attempt is made to separate it from the signal. For each mass hypothesis, the expected signal is known. The flat background is adjusted in order to build and maximise a likelihood probability. The probabilities corresponding to the known particle types are then used for tagging. For statistical analyses, the likelihood probability is computed as a function of the Cherenkov angle, and the best one retained $[47]$.
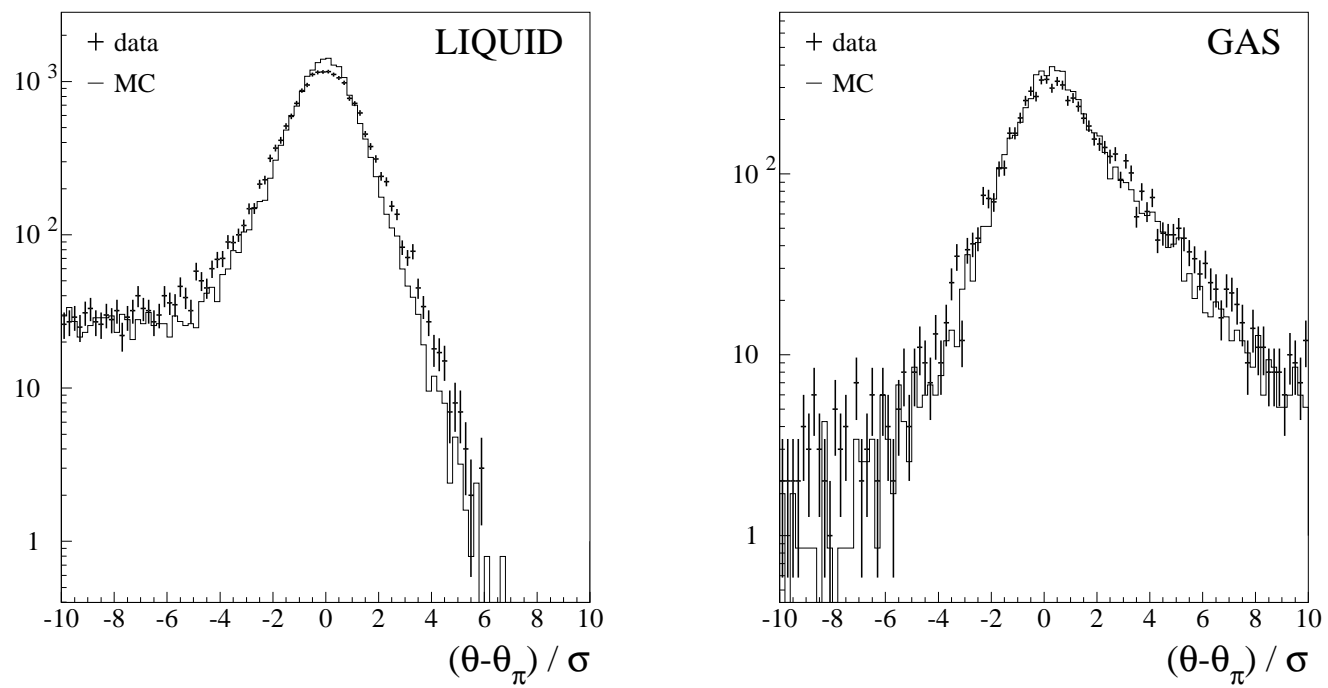

Figure 19: Average Cherenkov angle per track for pions from $\mathrm{K}^{0} \rightarrow \pi^{+} \pi^{-}$decays, relative to the pion hypothesis and normalized to the calculated width, for liquid (left) and gas (right) radiators.

The other approach (referred to as the "RIBMEAN" approach) uses a clustering algorithm to distinguish between background and signal photoelectrons. Photoelectrons are grouped into clusters which are weighted according to quality criteria, such as measurement errors or possible ambiguities between several tracks [48]. The best cluster is retained and weights used to measure the average Cherenkov angle, its error and the estimated number of photoelectrons. Quality flags are set to allow different rejection levels. They are based on the detector status and the cluster quality. Figure 19 shows the average angle distributions obtained in hadronic events, for data and simulation. The distribution of the average Cherenkov angle as a function of the momentum in multihadronic events, is shown in Figure 20 for the liquid (top) and gas (bottom) radiators.

Figures 21 and 22 illustrate the performances obtained for kaon and proton tagging in hadronic $Z$ decays, for sets of tagging cuts used in different analyses. In Figure 21 the emphasis has been put on the purity. In Figure 22 the requirement is a constant and high efficiency. The efficiencies are normalised to all tracks inside the geometrical acceptance of the detector. The classification probabilities for pions and protons have been checked 

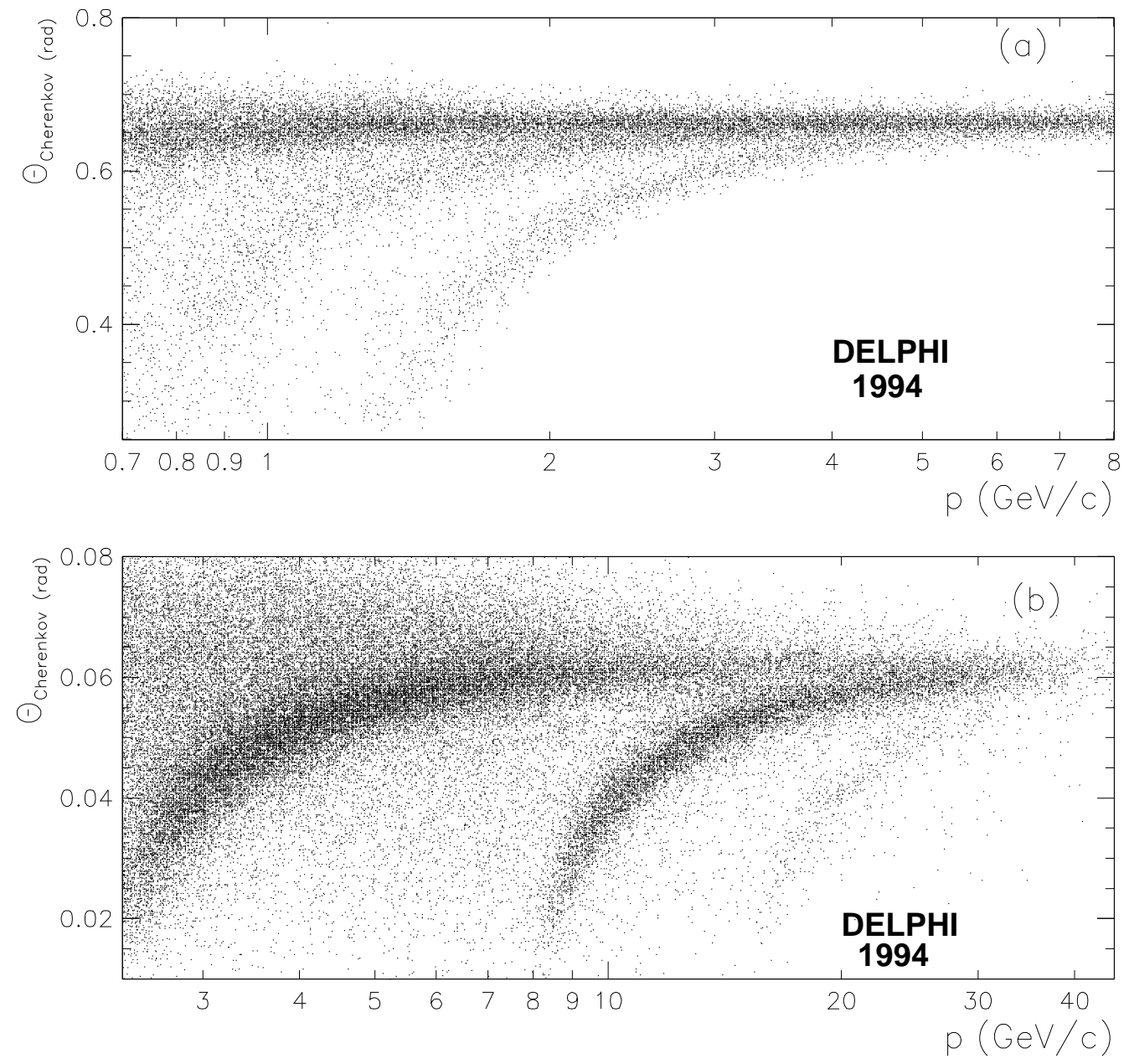

Figure 20: Average Cherenkov angle per track as a function of the momentum in multihadronic events in the Barrel RICH, for the liquid (top) and gas (bottom) radiators. The three bands on both plots correspond to pions (uppermost band), kaons (middle band) and protons (lowest band). 

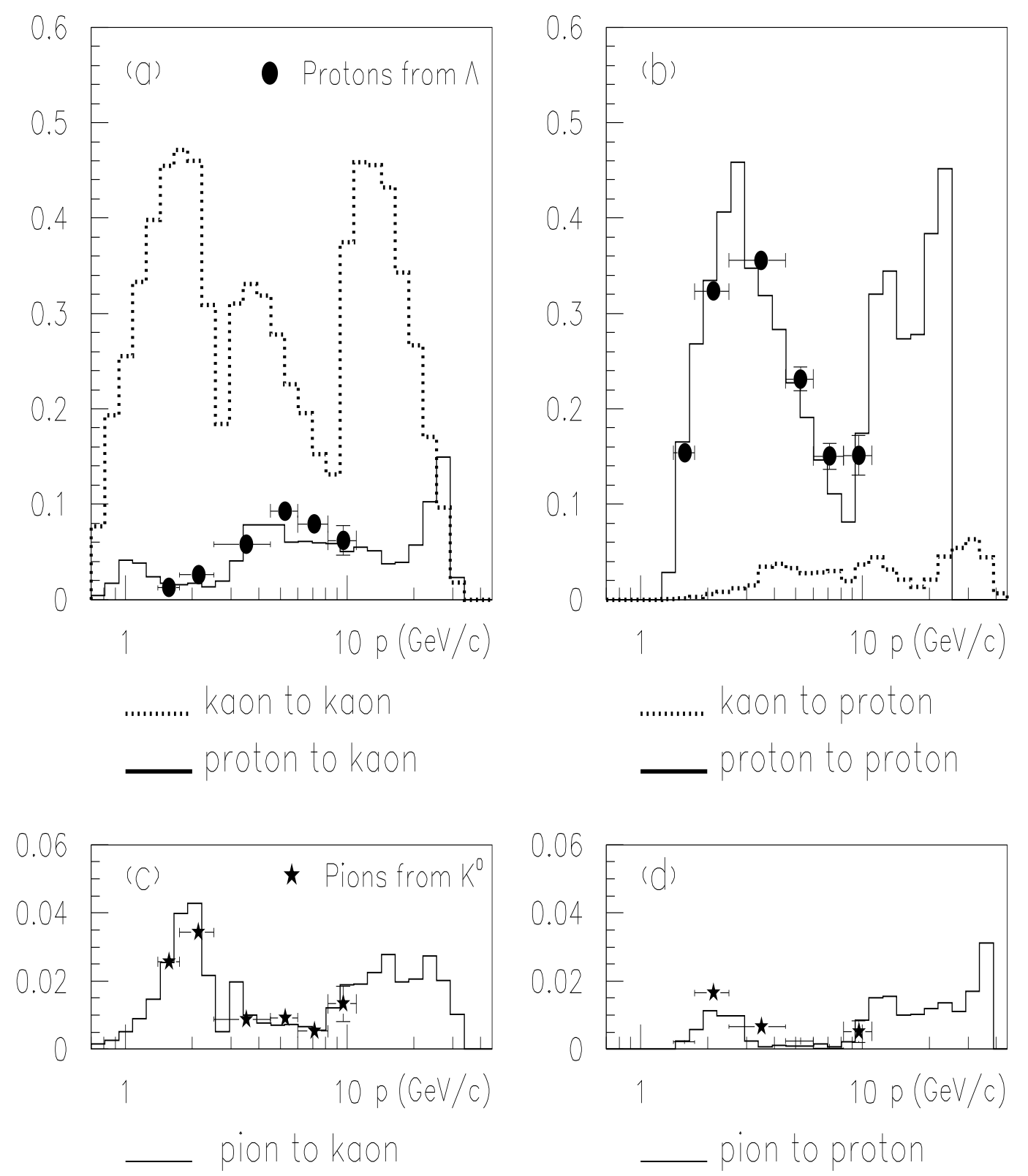

Figure 21: The performance of a high purity RICH tag (the "tight RIBMEAN" tag). (a) Fractions of protons (solid line) and kaons (dashed line) tagged as kaons in simulated hadronic decays as a function of momentum. The values found for protons from real $\Lambda^{0}$ decays are shown as circles. (b) Same as (a) but for particles tagged as protons. (c) Fraction of pions (solid line) tagged as kaons in simulated hadronic decays. The values found for pions from real $K^{0}$ decays are shown as stars. (d) Same as (c) but for particles tagged as protons. 

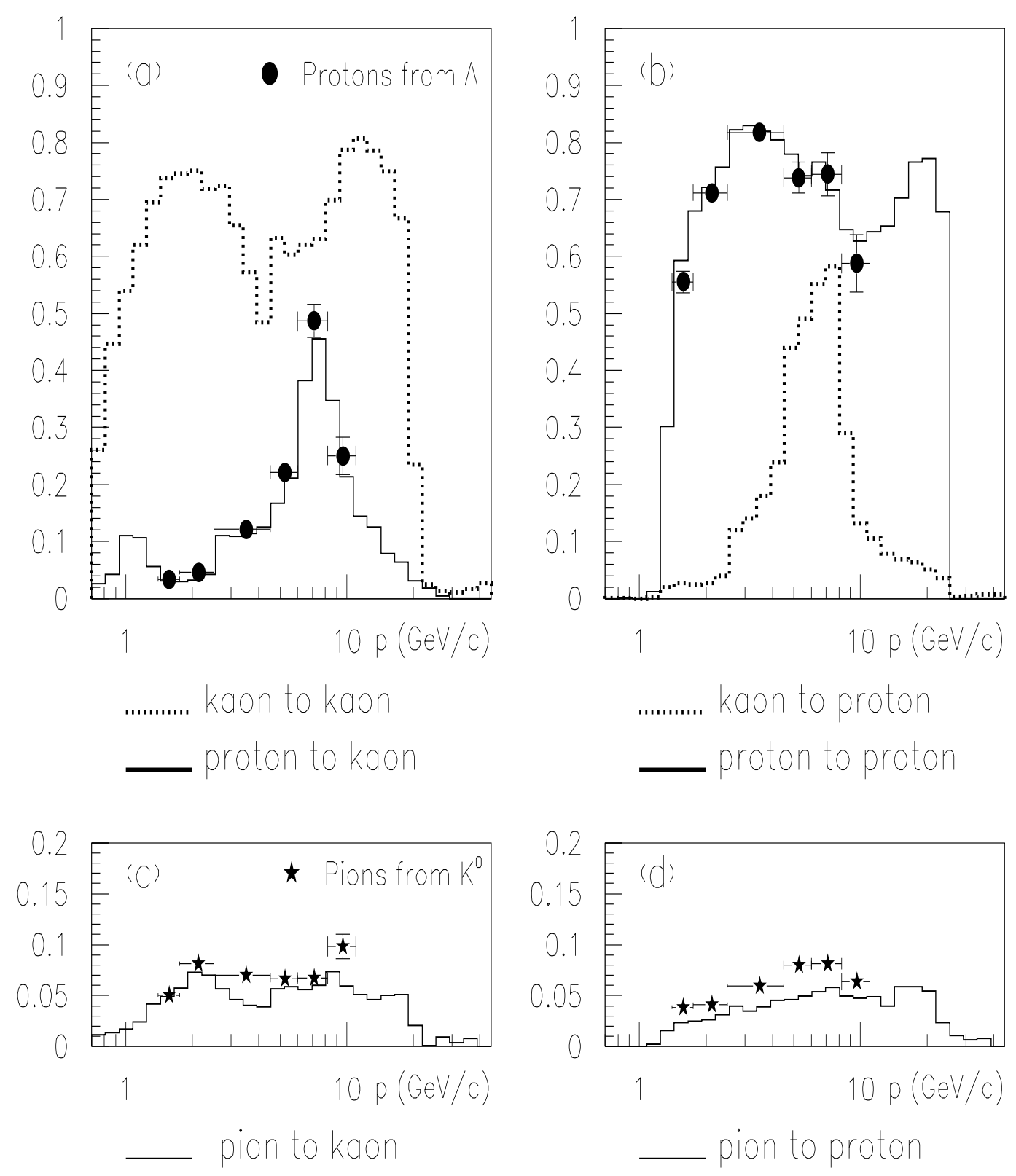

Figure 22: Same as Figure 21 but for a high efficiency RICH tag (the "loose HADSIGN" tag). The poorer agreement between real and simulated data reflects the fact that the HADSIGN software cannot be rerun at DST level (see Section 6). 
from the data (points with errors) using samples of pions from $\mathrm{K}_{S}^{0}$ decay and protons from $\Lambda$ decay respectively.

\subsection{Combining the Information}

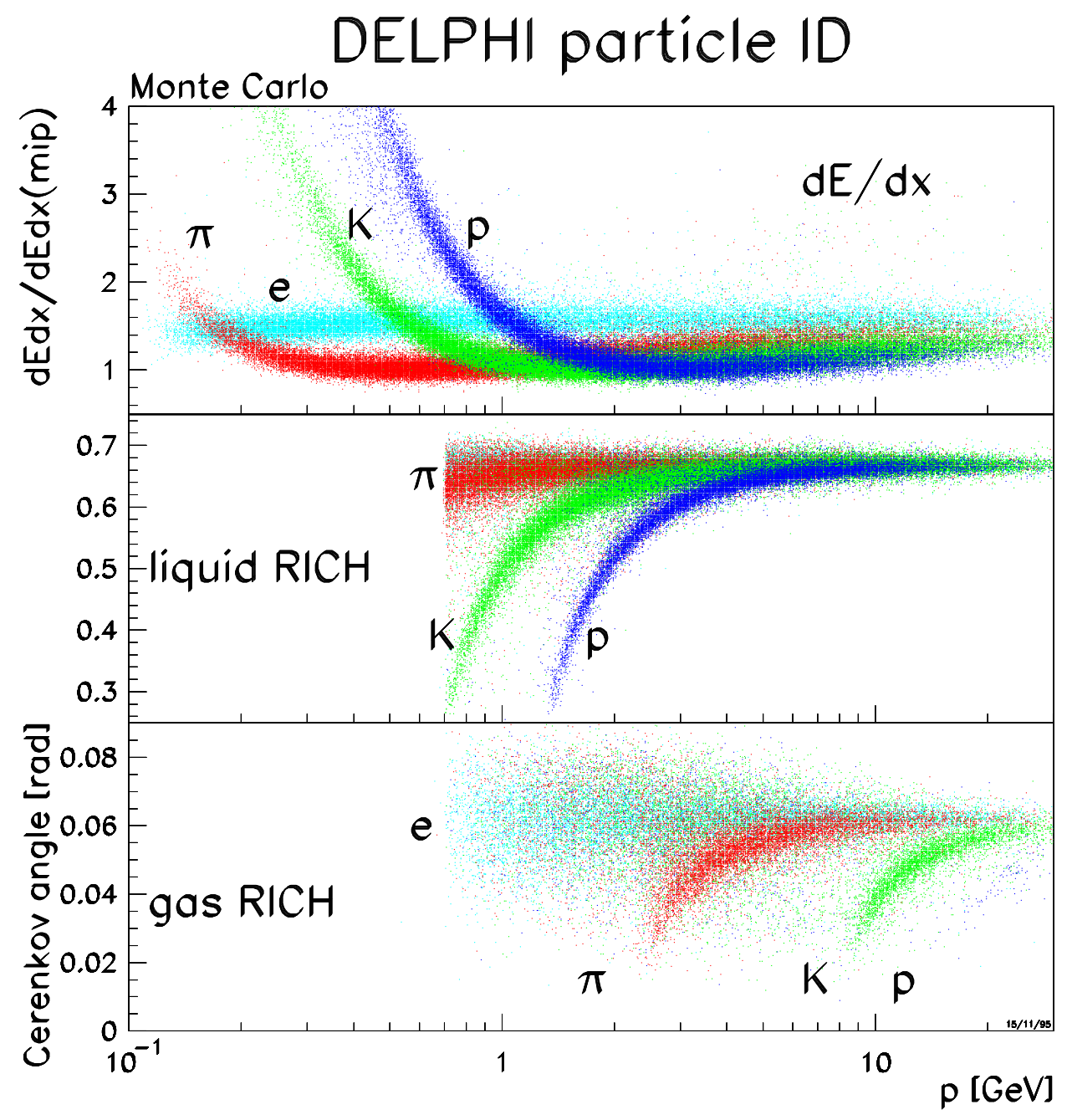

Figure 23: $d E / d X$ and RICH information for a set of simulated hadronic $\mathrm{Z}$ decays.

The $d E / d X$ and RICH data allow charged particle identification over most of the momentum range at LEP1 (Figure 23). They can be combined, providing three levels of proton and kaon tag (loose, standard and tight) corresponding to different purities. For example, the efficiency for the identification of a $\mathrm{K}^{ \pm}$using the standard tag, averaged over the momentum spectrum above $0.7 \mathrm{GeV} / c$, is about $70 \%$ with a contamination of $30 \%$. The typical efficiency for the identification of a proton is about $70 \%$ with a contamination of $50 \%$. Such a combined particle identification system can allow the unambiguous reconstruction of exclusive decays: a striking example is given in Figure 24 . 

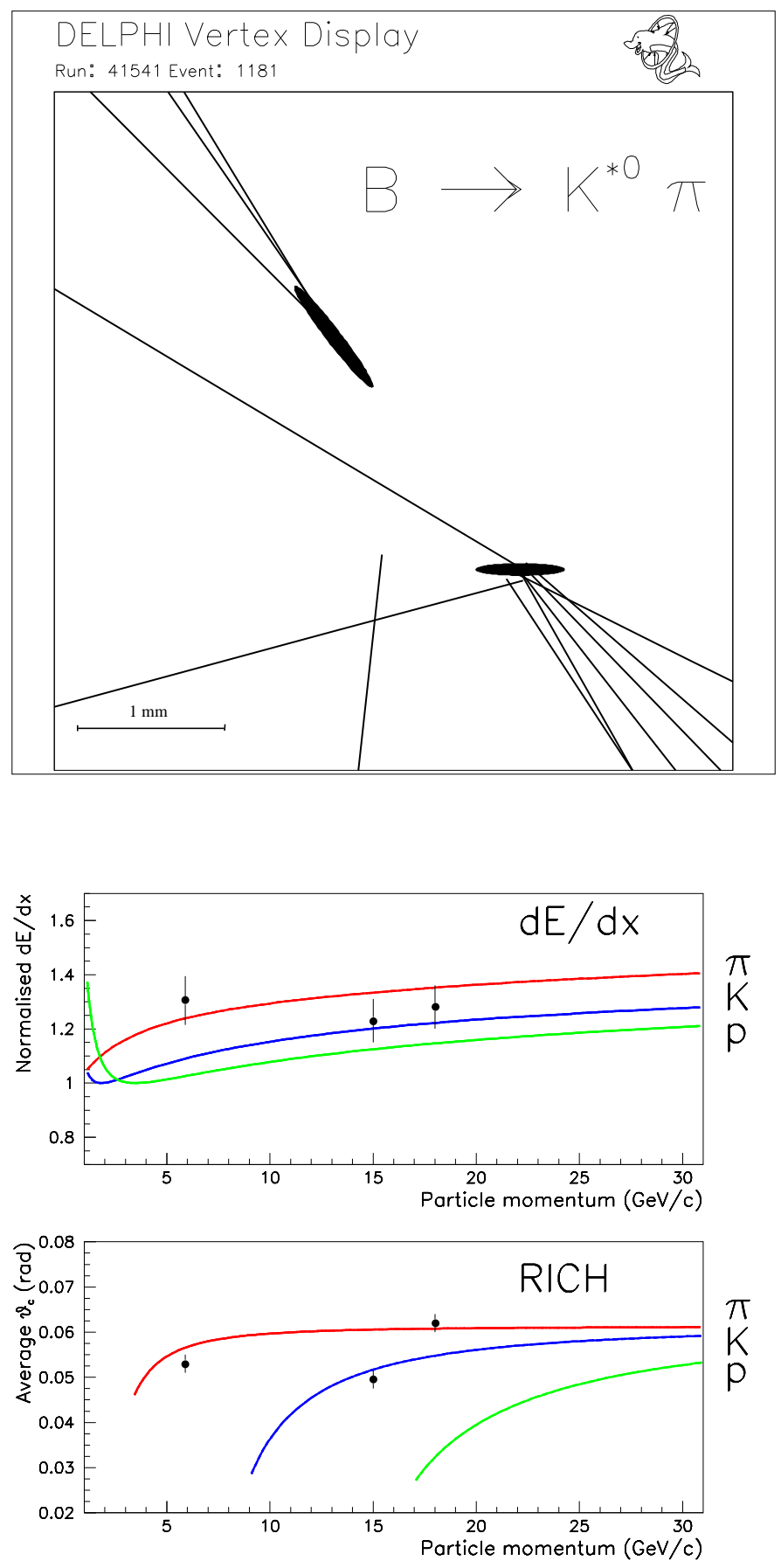

Figure 24: A candidate $B \rightarrow K^{*}(892) \pi$ decay. A magnified view of the tracks extrapolated to the vertex region in the $x y$ plane is displayed above: the $\mathrm{Z}$ decay (lower right) and $\mathrm{B}$ decay (upper left) vertices are indicated by error ellipses corresponding to $3 \sigma$ regions. The plots below summarise the hadron identification properties: the lines show the responses expected for pions, kaons and protons and the points with error bars the measured values for the reconstructed $B$ decay products. 


\section{Electromagnetic Calorimeters}

The electromagnetic calorimetry system of DELPHI is composed of a barrel calorimeter, the HPC, a forward calorimeter, the FEMC, and two very forward calorimeters, the STIC and the VSAT. The latter two are used mainly for luminosity measurement and were already described. There is no gap in angular coverage between the FEMC and the STIC. Supplementary photon taggers have been installed to cover the gap between the HPC and FEMC at $\theta \simeq 40^{\circ}$ and the $90^{\circ}$ and $\phi$ cracks in the HPC coverage ( i.e. between the HPC modules) not already covered by the TOF, thus establishing complete hermeticity.

\subsection{Barrel Electromagnetic Calorimeter}

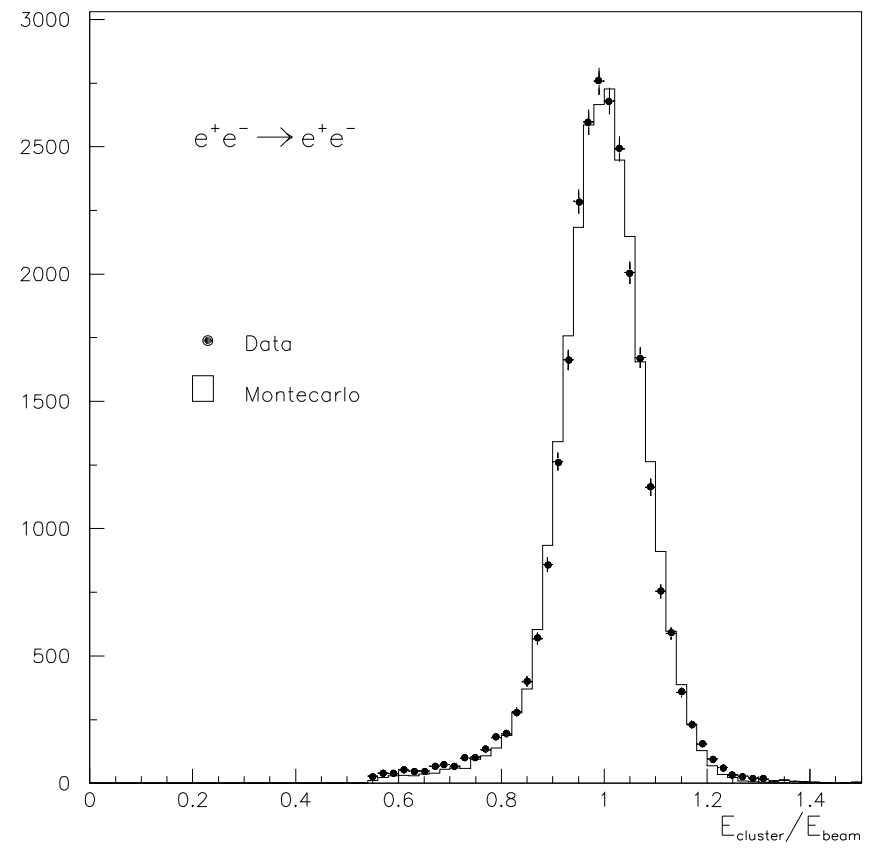

Figure 25: Distribution of the energies deposited in the HPC by Bhabha electrons in real and simulated data.

The barrel electromagnetic calorimeter, HPC [49], consists of 144 modules arranged in 6 rings inside the magnetic field. Each ring consists of 24 modules coaxially arranged around the beam axis with an inner radius of $208 \mathrm{~cm}$ and an outer radius of $260 \mathrm{~cm}$. Each HPC module is a small TPC with layers of high density material in the gas volume. These layers are made from lead wires which serve not only as converter material, but provide the drift field as well. The total converter thickness is $18 X_{0} / \sin \theta$. In each module there are 128 pads arranged in 9 rows. In the first row, nearest the beamspot, the pads are 2 $\mathrm{cm}$ wide, increasing to $8 \mathrm{~cm}$ wide in the last row. The charge of each pad is sampled in 256 time slots, providing very high granularity in $z$.

Equalisation of the energy response of the pads inside one module and first order module calibration and monitoring of ageing (which was found to cause a gain reduction of $0.12 \%$ per full day of running and is now fully compensated by raising the high voltages) are achieved using radioactive Krypton gas. Gas gain and drift velocity stability are 
monitored online using test chambers in the HPC gas circuit. In addition, pressure and temperature are monitored and corrected for in the reconstruction.

The first offline reconstruction step is to cluster the information from each single pad. Special care is taken to suppress fluctuations due to slow electrons curling in the magnetic field. The cluster reconstruction procedure reduces the contribution to the single cluster $z$-precision from the readout granularity to below $1 \mathrm{~mm}$.

Shower reconstruction then proceeds as follows. The charge strings of the accepted clusters of all nine layers are projected radially from the interaction point onto a $z-R \phi$ grid at a constant radius of $217 \mathrm{~cm}$ (corresponding to the third layer) of size $3.4 \mathrm{~mm} \times 3.4$ $\mathrm{mm}$ (corresponding to one timeslot in $z$ ). In the $R \phi$ direction, the charge measured in a pad is uniformly distributed among the grid bins covered by the pad. Then neighbouring bins are added into a coarser grid of $0.5^{\circ} \times 0.5^{\circ}$ resolution. On this grid a local maximum search is performed and connected areas are separated if a significant minimum is found between two local maxima. All bins that remain connected are collected into the shower defined by the nearest local maximum. Fits with removal of the tails are then performed in order to calculate a shower reference point (at a radius of $217 \mathrm{~cm}$ ) and internal shower directions.

In the second stage pattern recognition, charged particle tracks are extrapolated into the HPC and linked to HPC showers if they are compatible with the hypothesis of originating from the track. Also additional low energy showers may be reconstructed along the track extrapolation and, if necessary, showers may be split.

Final energy calibrations and alignment are performed using $\mathrm{Z} \rightarrow e^{+} e^{-}$events. Each electron track is extrapolated into the HPC and the $z$-coordinates of reconstructed clusters are compared to the track extrapolation. Mean drift velocity $\left(v_{D}\right)$ and time offset $\left(t_{0}\right)$ corrections are calculated for each module, as well as apparent $t_{0}$ and $v_{D}$ corrections for each layer and ring to account for the non-pointing geometry.

The reference point spatial resolutions achieved using this procedure are (for $45 \mathrm{GeV}$ electrons) $\sigma(z)=0.13 \mathrm{~cm}$ in the innermost rings (smallest $|z|), 0.22 \mathrm{~cm}$ in the middle rings and $0.31 \mathrm{~cm}$ in the outer rings. This corresponds to a nearly constant $\theta$ resolution of $0.6 \mathrm{mrad}$ for $45 \mathrm{GeV}$ electrons. The apparent $\phi$ resolution for electrons is $3.1 \mathrm{mrad}$, but much of this is due to the uncertainty in the electron track extrapolation from the TPC through the material of the RICH into the OD and the HPC. The energy resolution obtained for $45 \mathrm{GeV}$ electrons is about $6.5 \%$ (see Figure 25 ).

The linearity of the HPC energy response is monitored using neutral pions reconstructed with high precision from one photon converted before the TPC and one photon reconstructed in the HPC (see Section 13). The relative precision on the measured energy can be parametrized as $\sigma(E) / E=0.043 \oplus 0.32 / \sqrt{E}(E$ in $\mathrm{GeV})$ and the angular precisions for high energy photons are $\pm 1.7 \mathrm{mrad}$ in the azimuthal angle $\phi$ and $\pm 1.0 \mathrm{mrad}$ in the polar angle $\theta$.

\subsection{Forward Electromagnetic Calorimeter}

The Forward ElectroMagnetic Calorimeter, FEMC, consists of two arrays of 4532 Cherenkov lead glass blocks; the front faces are placed at $|z|=284 \mathrm{~cm}$, covering the polar angles $8^{\circ}<\theta<35^{\circ}$ and $145^{\circ}<\theta<172^{\circ}$. The blocks are truncated pyramids with inner (outer) face dimensions of $5.0 \times 5.0(5.6 \times 5.6) \mathrm{cm}^{2}$ and depths of $40 \mathrm{~cm}$, corresponding to 20 radiation lengths. Each block is mounted in the detector to point near to the interaction region. A tilt angle of $\sim 1^{\circ}$ was applied in order to avoid any particle escaping undetected in the insensitive regions between the blocks. The Cherenkov signal 
induced by the charged particles in the shower is read out by a single stage photomultiplier (triode) designed to operate inside the DELPHI magnetic field, coupled to a low noise preamplifier. The noise of the electronic readout chain in DELPHI is $\sim 35 \mathrm{MeV}$ per channel.

The reconstruction of electromagnetic showers is performed in two stages.

The first stage is an iterative search for energy clusters. In each endcap, the glass with the largest energy deposit not yet associated with others is located and the eight adjacent ones are taken as part of the cluster if their energies exceed a threshold value. A flag is set if a glass had previously been attributed to a contiguous cluster and its energy is then shared between the clusters in proportion to the energies in their central glasses. The cluster energy is then evaluated by summing the energies assigned to it and its coordinates are calculated from their centre of gravity. The energy sharings between contiguous clusters are then refined taking into account the computed energies and positions of the clusters. The process continues until all energies above threshold have been assigned to an energy cluster.

The second stage uses information from the tracking system to distinguish the clusters due to neutral particles from those coming from charged particles. A matching is performed based on a $\chi^{2}$ comparison between the predicted impact point and the reconstructed shower position. The efficiency of the matching algorithm is however degraded for electrons due to the emission of hard bremsstrahlung photons (more than one radiation length of material is crossed between the interaction point and the FEMC).

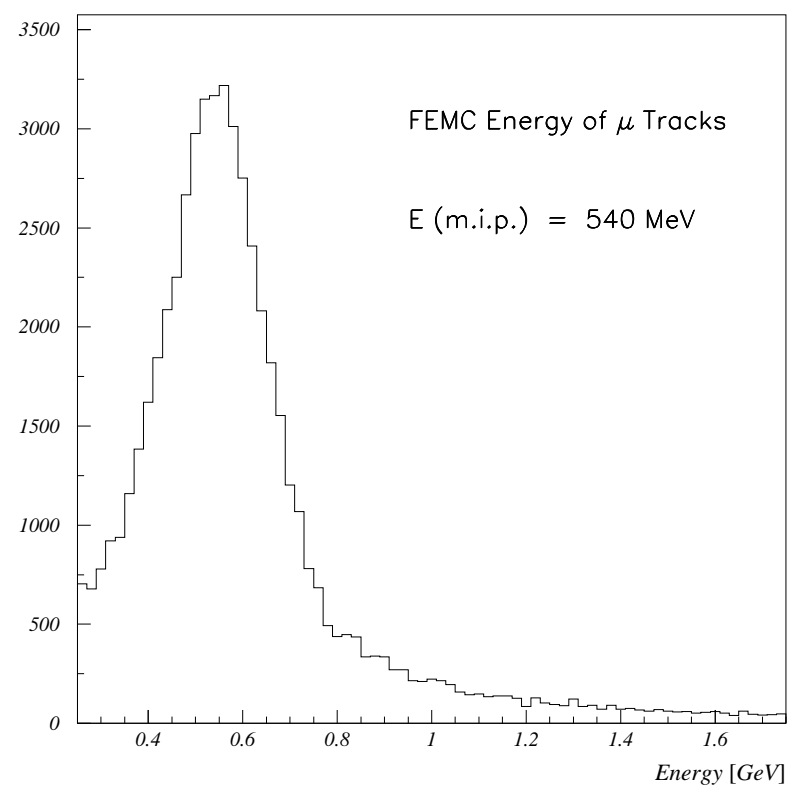

Figure 26: Distribution of the energies deposited in the FEMC by muons selected by means of the tight cuts, see Section 16.

Bhabha events are used to calibrate the detector. Clusters of 25 glasses are formed centred around the glass with the largest energy deposit. An iterative algorithm is then applied, aiming to minimize the spread of the energy about the nominal beam energy. The calibration constants were found to vary on average by less than $1 \%$ per year. This 
procedure calibrates about $90 \%$ of the detector, but in the region $\theta>32^{\circ}$ the electron energy is degraded too much by interactions in the TPC support structures. Counters in this region are therefore calibrated using muons, which deposit $540 \mathrm{MeV}$ in each glass with an energy spread of about $20 \%$ (see Figure 26).

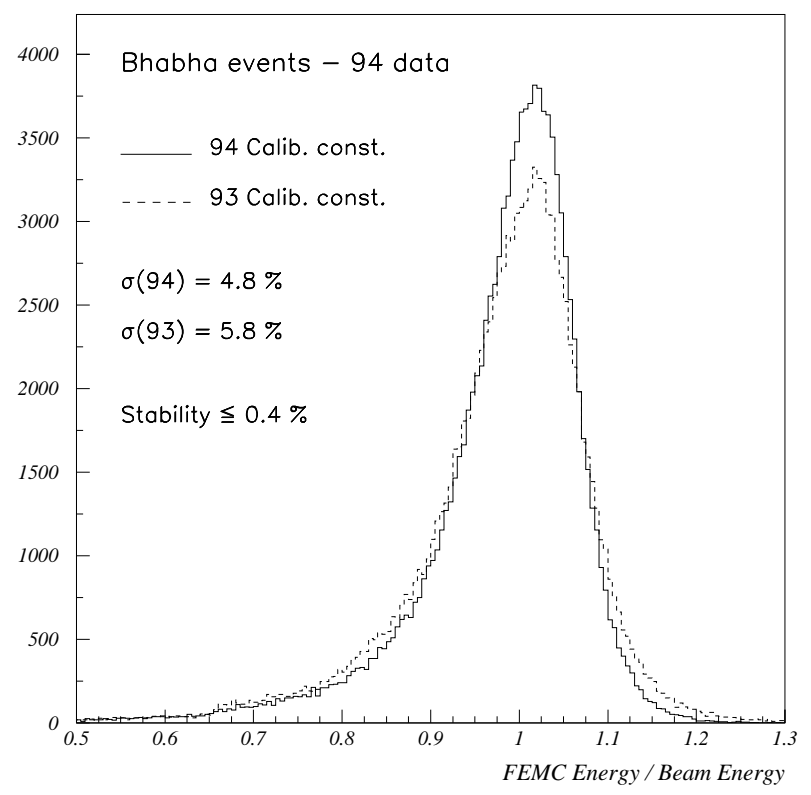

Figure 27: Energies of Bhabha electrons as seen by the FEMC, normalized to the beam energy. The energy distribution after the detector calibration is compared to that obtained using the calibration constants determined for the previous year.

The energy resolution for Bhabha electrons is $4.8 \%$ (see Figure 27), degraded as compared to the test beam results by pre-showering of the electrons in the material between the beam intersection point and the detector. The relative precision on the measured energy can be parametrised as $\sigma(E) / E=0.03 \oplus(0.12 / \sqrt{E}) \oplus(0.11 / E)$ where $E$ is in $\mathrm{GeV}$. For neutral showers of energy larger than $2 \mathrm{GeV}$, the average precision on the reconstructed hit position in $x$ and $y$ projected to $|z|=284 \mathrm{~cm}$ is about $0.5 \mathrm{~cm}$.

\subsection{Supplementary Photon Taggers}

At LEP2, in order to be sensitive to possible new physics whose experimental signatures are based on missing energy and momentum in the event, a hermetic detector is needed. For this purpose various sets of lead-scintillator counters have been placed in the uncovered regions at $\theta \simeq 90^{\circ}$ and $\theta \simeq 40^{\circ}[50]$.

The TOF counters are also used to provide information for those particles (mainly photons) that go in the dead regions of the inner-most detector layers of DELPHI. It has been observed that $84 \%$ of $45 \mathrm{GeV} / \mathrm{c}$ muons are detected by one TOF counter. As shown in Figure 28, the detection efficiency in the TOF for electrons and photons depends on the shower energy. For energies above $10 \mathrm{GeV}$, the efficiency averaged over all angles is about $90 \%$ but, more importantly, around the HPC cracks the efficiency is close to $100 \%$. 


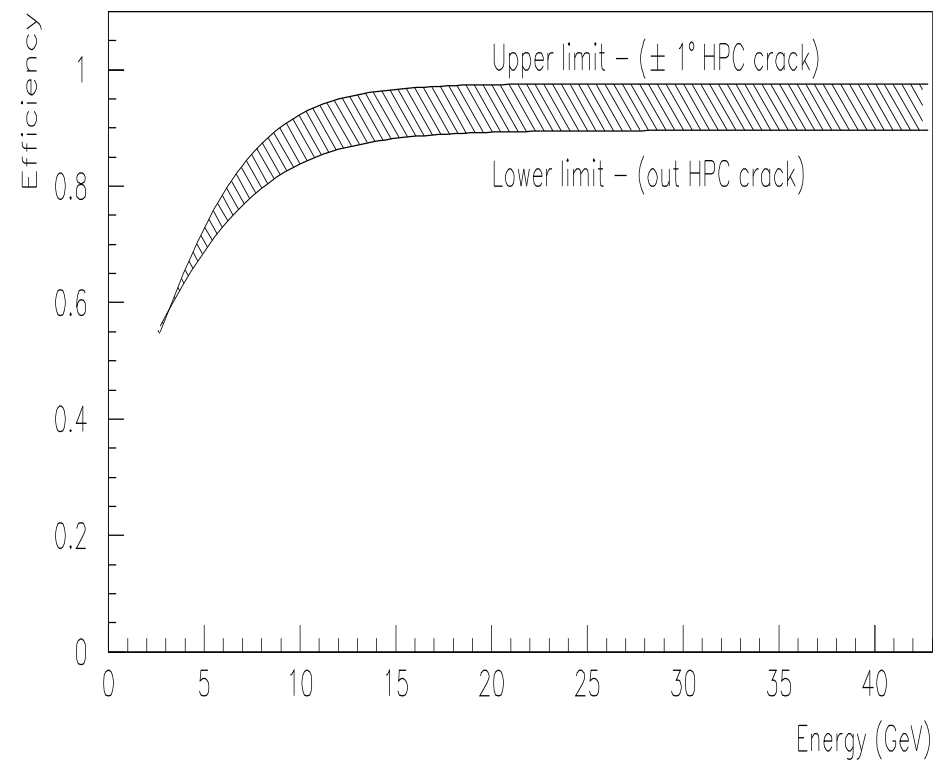

Figure 28: Efficiency for detecting electrons and photons in the TOF as a function of the shower energy. The lower and upper bounds reflect the results obtained when all regions (lower bound) or only the region covering the HPC cracks (upper bound) are considered.

The TOF does not fully cover two $\phi$ regions obstructed by the supports of the coil. These regions are therefore covered by lead-scintillator counters (" $\phi$ taggers") that are placed directly in the $\phi$ cracks between the HPC modules.

\section{Electron Identification}

Identification of electrons is complicated by electromagnetic interactions in front of the calorimeters. In the barrel region, the material amounts to about $0.8 X_{0} / \sin \theta$. In the forward region it is larger and the material is on average further from the electromagnetic calorimeter.

Electron identification in the barrel part of DELPHI is performed using two independent and complementary measurements, the $d E / d X$ measurement of the TPC and the energy deposition in the HPC. Probabilities from calorimetric measurements and tracking are combined to produce an overall probability for the electron hypothesis.

The comparison of the energy $E$ in the calorimeter with the independently measured momentum $p$ from the tracking devices provides a powerful tool for electron identification. After correction for radiation effects in front of the calorimeter and for small nonlinearity effects inside the calorimeter, the ratio $E / p$ is expected to be close to unity independent of the electron energy. The $E / p$ distribution is parametrised and converted into a probability for the electron hypothesis.

Charged particle tracks are extrapolated from the TPC to the calorimeter and their crossing point with the HPC as well as their directions are calculated. The comparison of these values with the position and direction measurement of the shower in the HPC leads 
to additional $e-\pi$ separation. The most powerful tools were found to be $\Delta z$ (position mismatch in $z$ ) and $\Delta \phi$ (direction mismatch in $\phi)$.

In order to quantify the electromagnetic character of a shower profile, the longitudinal shower shape is parametrised using a $\Gamma$-distribution for the energy deposition rate $d E / d t$ as a function of the shower depth $t$ in radiation lengths:

$$
\frac{d E}{d t}=E \cdot \beta \cdot \frac{(\beta t)^{\alpha-1} \cdot e^{-\beta t}}{\Gamma(\alpha)},
$$

where $E$ is the shower energy, and $\alpha$ and $\beta$ are empirical parameters determined from the data, and are functions of the shower energy $E$. To a good approximation the dependence of $\alpha$ and $\beta$ on the angles $\theta$ and $\phi$ from the track extrapolation to the HPC can be neglected. The shower depth $t$ can be calculated by taking into account the $\theta-$ and $\phi$-dependent geometrical factors for the material distribution in front of the HPC and the correct material distribution inside the HPC. Performing a three parameter fit $\left(E, \alpha\right.$ and $\beta$ ) leads to the definition of a $\chi^{2}$-like variable to quantify the electromagnetic character of a shower profile.

The second completely independent piece of information that was used to distinguish between electrons and hadrons is the $d E / d X$ measurement in the TPC. The value of the Fermi plateau, normalized to the minimum ionizing particle, is found to be 1.52 units. For isolated particles (muons in dimuon events) the root mean square resolution on the mean $d E / d X$ is $5.5 \%$, while for particles in jets the root mean square resolution is $7.4 \%$ (see Section 10.1). Thus the separation between $e$ and $\pi$ in jets is above $3 \sigma$ for momenta below $4.5 \mathrm{GeV} / c$ and above $2 \sigma$ for momenta up to $20 \mathrm{GeV} / c$.

The electron identification in the forward part of DELPHI is also based on cuts on the ratio $E / p$ of the electromagnetic energy associated to a track and the track momentum. For this purpose all tracks in the forward region fulfilling certain quality criteria are extrapolated to the FEMC. Showers are then associated to the tracks taking into account the specific spread of energy created by electrons. The $d E / d X$ information provided by the TPC is also used, but to a smaller extent than in the barrel region

Throughout, three different levels of tagging are provided; they can classify electrons above a momentum of $2 \mathrm{GeV} / c$. Their typical efficiencies and misidentification probabilities are shown in Table 8; note that there are on average about 21 charged particles per event, about 17 of which are pions [51]. Figure 29 illustrates the efficiency and purity of the DELPHI electron identification (standard tag) as a function of track momentum $p$ and polar angle $\theta$.

\begin{tabular}{|l|c|c|}
\hline Tag & Efficiency (\%) & Misid. Probability (\%) \\
\hline Loose & 80 & $\simeq 1.6$ \\
Standard & 55 & $\simeq 0.4$ \\
Tight & 45 & $\simeq 0.2$ \\
\hline
\end{tabular}

Table 8: Efficiencies and misidentification probabilities (measured with $\mathrm{K}_{S}^{0} \rightarrow \pi^{+} \pi^{-}$) for the electron sample $(p>3 \mathrm{GeV} / c)$ in multihadronic events.

Electrons passing through material lose energy by radiating soft and hard photons. Consequently the electron trajectory is not a perfect helix, but a spiral with decreasing radius of curvature. The association of HPC showers to electron tracks has to account for this effect. 

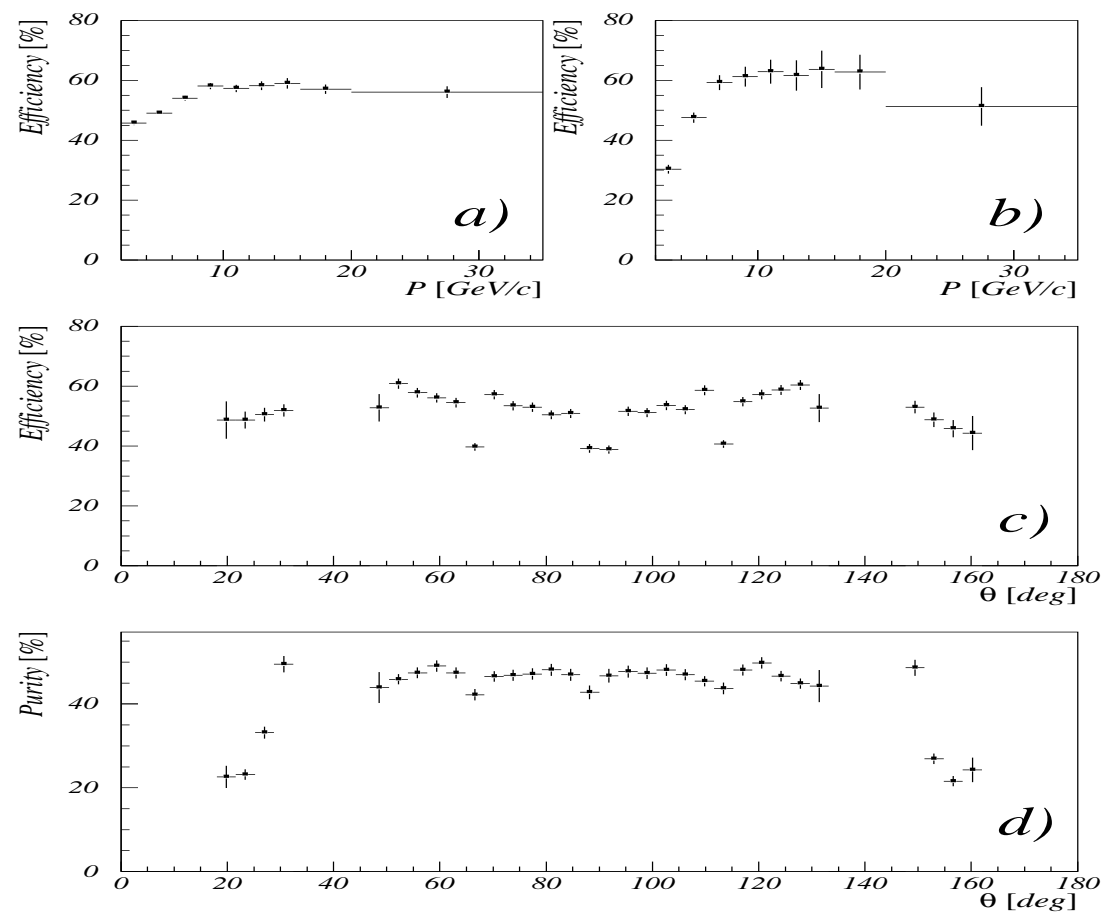

Figure 29: The upper plots show the electron identification efficiency (standard tag) as a function of momentum in the barrel region (a) and the forward region (b). The lower plots show the efficiency (c) and purity (d) as a function of the polar angle $\theta$. 
The reconstruction software looks for radiation of electron candidates in front of the TPC by a very simple procedure: tracks which are electron candidates (loose, standard or tight electron tag) are extrapolated to two radii, namely $35 \mathrm{~cm}$ on the large $R$ side and to the radius of the first measured point minus $3 \mathrm{~cm}$ on the internal side; the likelihood of reconstructed photons to come from electron radiation between these points is then checked. If a photon is consistent with coming from collinear radiation, it is accepted as having been radiated off the track.

The tracks of all the electron candidates are then refitted omitting information from the OD (because of the large amount of material before it, particularly in the RICH) and any photons accepted as having been radiated off it are used to correct its momentum ${ }^{\ddagger}$.

\section{Photon and $\pi^{0}$ Identification}

About $40 \%$ of the photons convert before they reach the HPC. About $7 \%$ of the photons convert in front of the TPC creating visible $e^{+} e^{-}$pairs, and a useful fraction of these can be reconstructed very precisely. This is achieved using algorithms which aim for a reconstruction of first order radiation effects (the calculations explicitly assume that opening angles are zero). The reconstructed converted photons have an energy precision of $\pm 1.2 \%$, and a directional precision of $\pm 1.5 \mathrm{mrad}$ in $\theta$ and $\phi$, and a precision on the conversion radius of $\pm 5 \mathrm{~mm}$.

Photon conversions in the outer wall of the TPC and in the RICH are not reconstructed due to the limited charged particle tracking.

Photon showers in the HPC and FEMC are reconstructed by summing neighbouring clusters (see Sections 11.1 and 11.2). If the width of a cluster is large, i.e. it spans several drift time intervals, a higher threshold is applied, and one or more new clusters are found. A shower is assumed to be caused by a neutral particle if it cannot be associated to a track.

A $\pi^{0}$ can be reconstructed either by pairing photons (converted before the TPC or seen in the calorimeters), or by analysing energy depositions in the calorimeters that display an internal structure.

\section{1 $\pi^{0}$ Reconstruction from Single Photons}

Having reconstructed photons converted before the TPC and in the HPC, $\pi^{0}$ 's can be searched for by calculating the invariant $\gamma \gamma$ mass [52]. Combining converted and HPC photons gives three different $\pi^{0}$ reconstruction methods: pairs of converted photons, pairs of one converted photon and one HPC photon (which are used for low and intermediate $\pi^{0}$ energies, typically from 0.5 to $15 \mathrm{GeV}$ ), and pairs of HPC photons which are used in the $\pi^{0}$ energy range from 3 to $8 \mathrm{GeV}$. Figure $30(\mathrm{a})$ shows the invariant mass spectrum of the combination of two converted photons, Figure $30(\mathrm{~b})$ from the combination of a converted photon with a HPC photon and Figure 30(c) from the combination of two HPC photons in the energy ranging from 4 to $8 \mathrm{GeV}$. In each case there is a clear $\pi^{0}$ signal on top of a combinatorial background.

The position and width of the $\pi^{0}$ peak for the sample containing one conversion photon and one HPC photon offers an excellent opportunity to understand the systematics of the HPC at low energies, since (as Figure 30a shows) the converted photons are very well measured. Although the number of converted photons is an order of magnitude less

\footnotetext{
${ }^{\ddagger}$ However no correction or error contribution is presently added to allow for soft bremsstrahlung.
} 

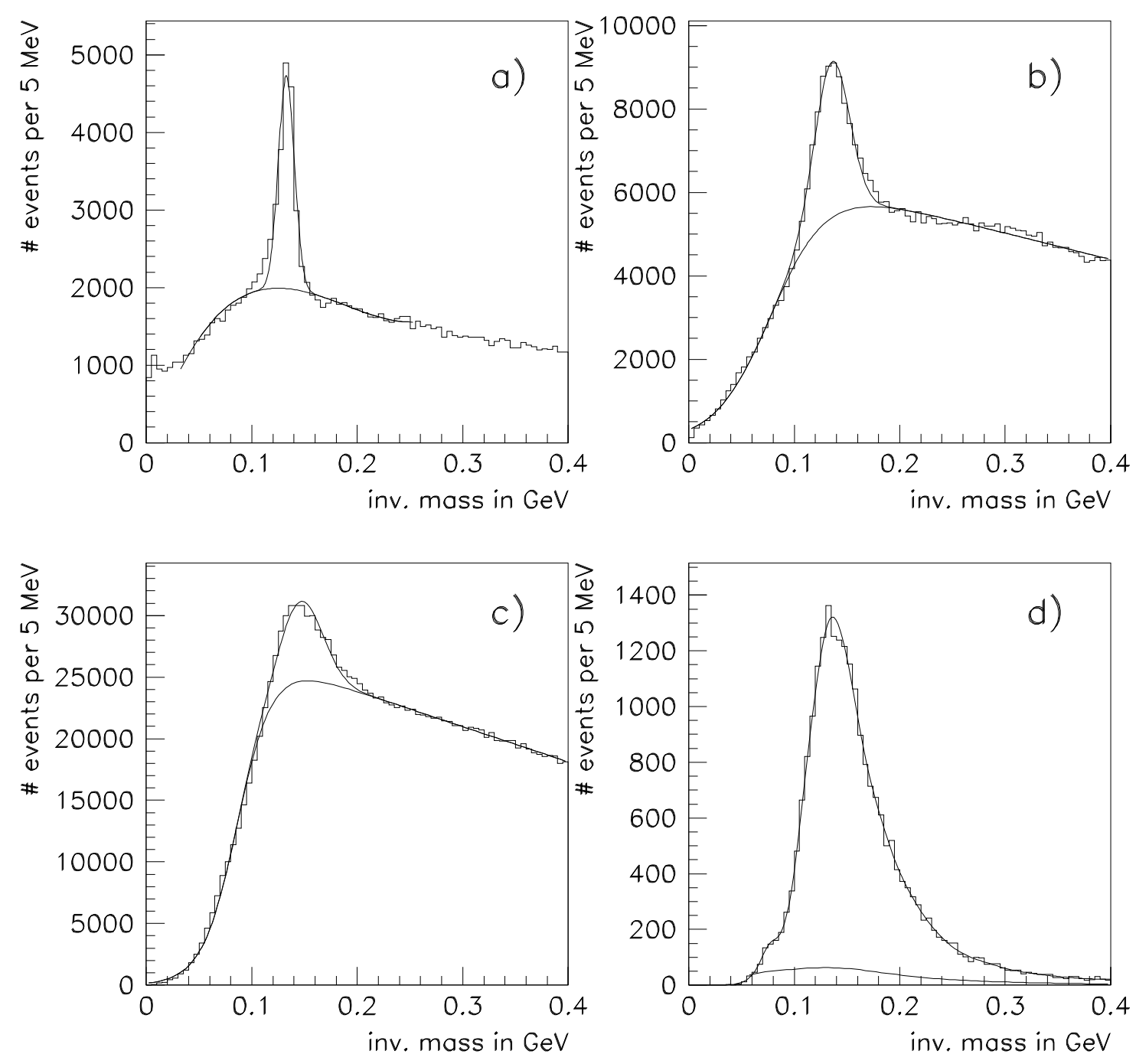

Figure 30: Invariant mass spectra for the four different $\pi^{0}$ reconstruction methods: (a) combination of two converted photons; (b) combination of a converted photon with an HPC photon; (c) combination of two HPC photons in the energy range from 4 to $8 \mathrm{GeV}$; (d) mass calculated from merged HPC showers in the energy ranging from 10 to $25 \mathrm{GeV}$. In $(\mathrm{a}, \mathrm{b}, \mathrm{c})$ the solid lines represent fits to the data; in (d) the background cannot be determined from the data so it was taken from the simulation. 
than that of HPC photons, the statistics are nevertheless large enough to provide a good estimate of the systematic errors.

The $\pi^{0}$ detection efficiency, as determined from the simulation for the 1992 and 1993 data, is shown in Figure 31 as function of the fractional momentum $x_{p}$. The efficiency is calculated as the number of reconstructed $\pi^{0}$ s in an $x_{p}$ bin after the photon selection cuts mentioned above, divided by the total number of generated $\pi^{0}$ 's in the same bin and the same geometrical acceptance $(|\cos \theta|<0.65)$. The efficiency for the $\pi^{0}$ reconstruction from two converted photons (one converted, one HPC photon) is about a factor 1.25 (1.10) larger in 1994, due to improvements in the track pattern recognition. These numbers include the fraction of photons converting, the efficiency of reconstructing conversions and HPC showers, and of identifying them as photons.

\section{2 $\pi^{0}$ Reconstruction in Merged Showers}

The angle between the photons from the decay of a $\pi^{0}$ with an energy above $6 \mathrm{GeV}$ is generally below $2^{\circ}$. In this case, the HPC pattern recognition program often reconstructs single or overlapping showers. To identify $\pi^{0}$ 's in HPC showers with reconstructed energies above $6 \mathrm{GeV}$ a lateral substructure is searched for by taking advantage of the very fine granularity of the HPC. The sampling width in the drift direction $(z)$ corresponds to $3.7 \mathrm{~mm}$, whereas the typical pad width in the first few layers is 2 to $3 \mathrm{~cm}$. In the substructure algorithm all cluster measurements are projected onto a $\theta$ - $\phi$ grid. A $\theta$-bin is chosen to correspond to a time slot. The charge of each $\theta$-bin of a pad is equally distributed into $\phi$-bins of the same size. The charges of all pads belonging to the shower are added up using a weight depending on the depth of the pad row. The weights were optimized in order to achieve the best two-shower separation, i.e. the pad-rows containing the start of the shower evolution get the highest weights.

The next step consists in finding the eigenvalues and eigenvectors of the lateral charge distribution tensor. The charge distribution is then projected onto the main axis and two Gaussians are fitted to this distribution. When there are two significant maxima, the showers are mainly from $\pi^{0}$ decay. The main background is from single photons that convert in the material just before the HPC. These also lead to two-cluster topologies. However, they are separated by the solenoidal magnetic field only in $\phi$, not in $\theta$. Therefore merged showers are rejected if the separation in $\theta$ between the shower centres is less than one-tenth of their separation in $\phi$. This cut removes $60 \%$ of the background.

The invariant mass of the charge distribution is calculated from the energy sharing between the two peaks and the distance between them. Studies using simulation show that both the reconstructed opening angle and the reconstructed energy depend slightly on energy. This can be explained by the features of the pattern recognition algorithm (maximal size of a shower) and the binning effects in the pad direction; corrections are made for these effects. Figure 30(d) shows the invariant mass for merged showers for energies larger than $10 \mathrm{GeV}$.

The background in the merged shower sample arises mainly from $\eta$ decays and varies with energy: at $6 \mathrm{GeV} 83 \%$ of the merged showers originate from $\pi^{0}$ 's, while at $10 \mathrm{GeV}$ the purity rises to $90 \%$. The combinatorial background is strongly reduced at these energies, since the algorithm only looks for the closest shower in space in a small cone of halfopening angle less than $2^{\circ}$, whereas the average distance to the nearest reconstructable uncorrelated photon is much larger. The detection efficiency of the algorithm has been determined by simulation and is shown in Figure 31(d). The tail in Figure 30(d) towards 

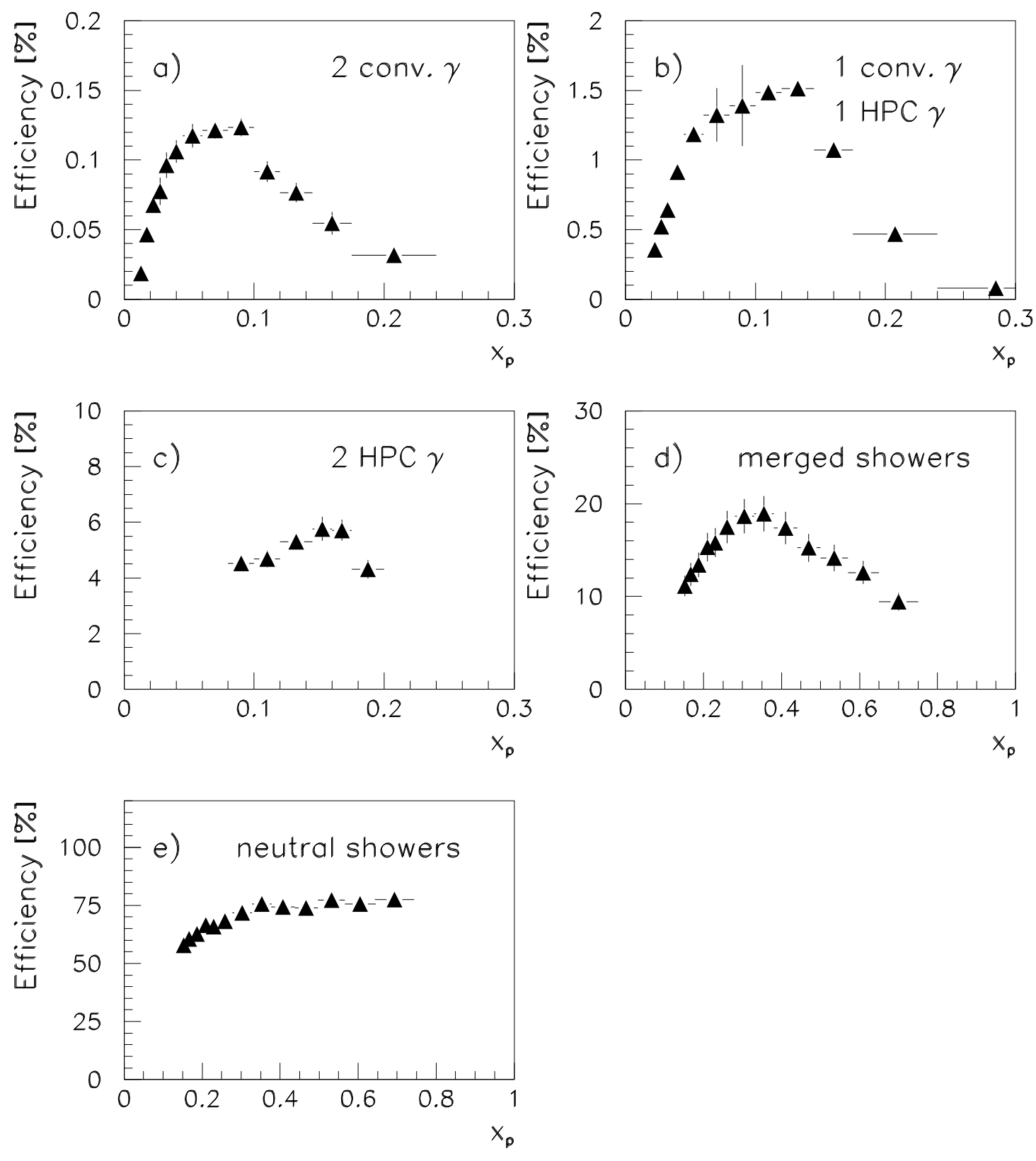

Figure 31: Efficiencies (for $|\cos \theta|<0.65$ ) as a function of the fractional momentum $x_{p}$ determined from the simulation for the five different $\pi^{0}$ reconstruction methods: (a) combination of two converted photons; (b) combination of a converted photon with a HPC photon; (c) combination of two HPC photons; (d) merged HPC showers; (e) single HPC showers not linked to a charged particle track. 
larger masses is due mainly to $\pi^{0}$ 's in which one of the photons converted just before the HPC.

\subsection{Single $\pi^{0}$ Showers}

For high energy $\pi^{0}$ s the opening angle between the photons is often too small for them to be resolved as two showers even in the HPC. As most photons come from $\pi^{0}$ decays, most of the energetic showers in the HPC are expected to be merged $\pi^{0}$ showers. One can therefore take as a $\pi^{0}$ any high energy shower in the HPC that is not associated to a charged particle. The purity obtained with this method is around $75 \%$ for energies from 6 to $25 \mathrm{GeV}$ and then it decreases slowly. The contamination is mainly from $\eta$ decays. The efficiency, calculated as the number of reconstructed showers after the photon selection cuts divided by the total number of generated $\pi^{0}$ 's in the same geometrical acceptance, is around $55 \%$ at $6 \mathrm{GeV}$ and rises gently up to $75 \%$ at $25 \mathrm{GeV}$, as shown in Figure $31(\mathrm{e})$.

\section{Hadron Calorimeter}

The HCAL is installed in the return yoke of the DELPHI solenoid. It is made of 2 endcaps, each consisting of 12 sectors, and a barrel section which consists of 24 modules. The whole Hadron Calorimeter covers almost the full solid angle: $11^{\circ}<\theta<169^{\circ}$. More than 19,000 limited streamer tubes $(8 \mathrm{~cm}$ in width, and varying in length between 40 to $410 \mathrm{~cm}$ ) are installed in the $18 \mathrm{~mm}$ wide slots between the $50 \mathrm{~mm}$ thick iron plates.

The limited streamer tubes are mounted on copper clad readout boards on which are scored up to 64 pads each covering a fixed angular region of $\Delta \phi=3.75^{\circ}$ and $\Delta \theta=2.96^{\circ}$. In the barrel part, five pads in the radial direction, called a tower, are read out together by the same electronic channel. In part (about 20\%) of the endcap, a tower is formed by seven pads, in the rest by four pads. The charge in each tower is integrated during $2 \mu \mathrm{s}$ and afterwards digitized by an 8-bit ADC.

The detector has proven to be very stable and reliable. Only around 150 streamer tubes out of the 19,000 have broken down, mostly during transport and installation in the pit. Starting at the beginning of the experiment in 1989 with $3.7 \mathrm{kV}$, the high voltage has been increased slowly and for several years now it has run very stably at $4.0 \mathrm{kV}$.

Muons produced in $\mathrm{Z} \rightarrow \mu^{+} \mu^{-}$decays are used for calibration. They have only $2 \%$ contamination from the $\tau^{+} \tau^{-}$channel and give a clean sample of penetrating particles. Hadronic showers are also used to set the energy scale. Calibration coefficients can be determined for each channel using muons and the azimuthal symmetry of the total energy deposited in a sample of hadronic $Z$ decays. The tower information is reconstructed in the form of clusters. Figure 32 shows the energy deposited in the HCAL by muons from $Z^{0} \rightarrow \mu^{+} \mu^{-}$, and Figure 33 shows the total energy deposited in hadronic $Z$ decays (ignoring neutral showers). The calibration for hadronic showers is checked using pions from single-prong $\tau$ decays that penetrate the electromagnetic calorimeter. In the barrel region $\left(52^{\circ}<\theta<128^{\circ}\right)$ the energy precision in the hadron calorimeter is found to be

$$
\sigma(E) / E=0.21 \oplus 1.12 / \sqrt{E}
$$

(with $E$ expressed in $\mathrm{GeV}$ ). The fixed term in this expression is due to the material between the hadron calorimeter and the electromagnetic calorimeter. Figure 34 illustrates the energy resolution of the hadron calorimeter for two momentum intervals for charged particles that have any energy deposition in HCAL. It shows the ratio of the energy 


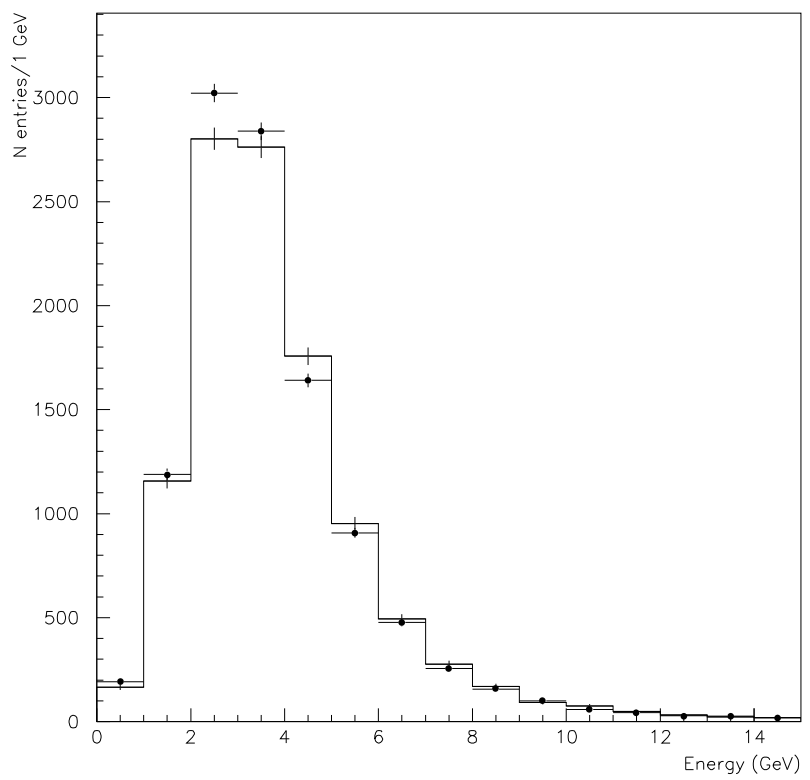

Figure 32: Energy in HCAL deposited by muons identified in the muon chambers. Real data (dimuons from the 1994 run) are shown by the points and simulation by the histogram.

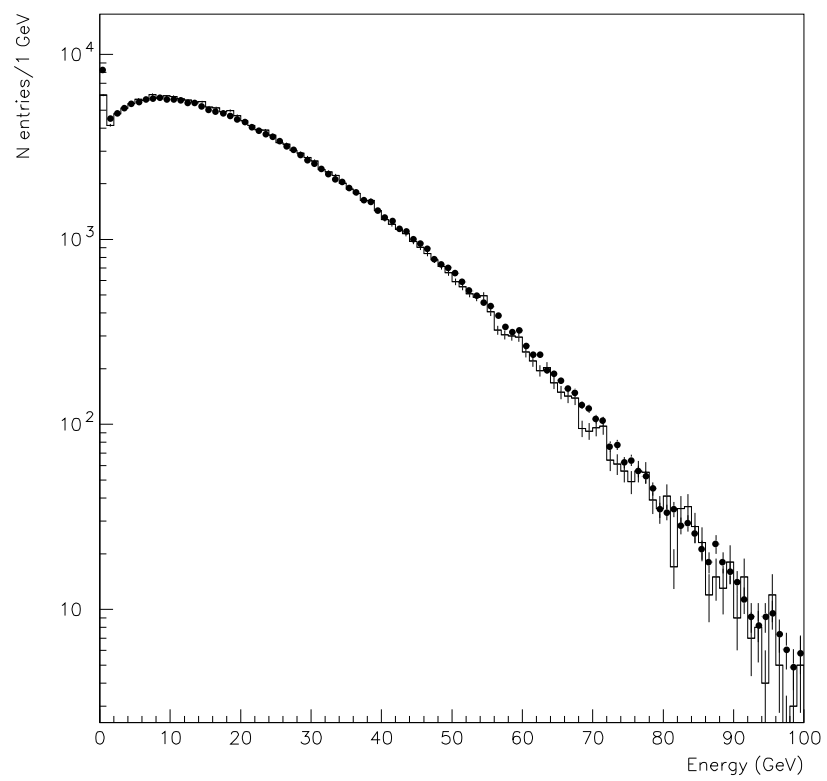

Figure 33: Total energy deposited in the HCAL in hadronic Z decays. Data (from the 1994 run) are shown by the points and simulation by the histogram. 

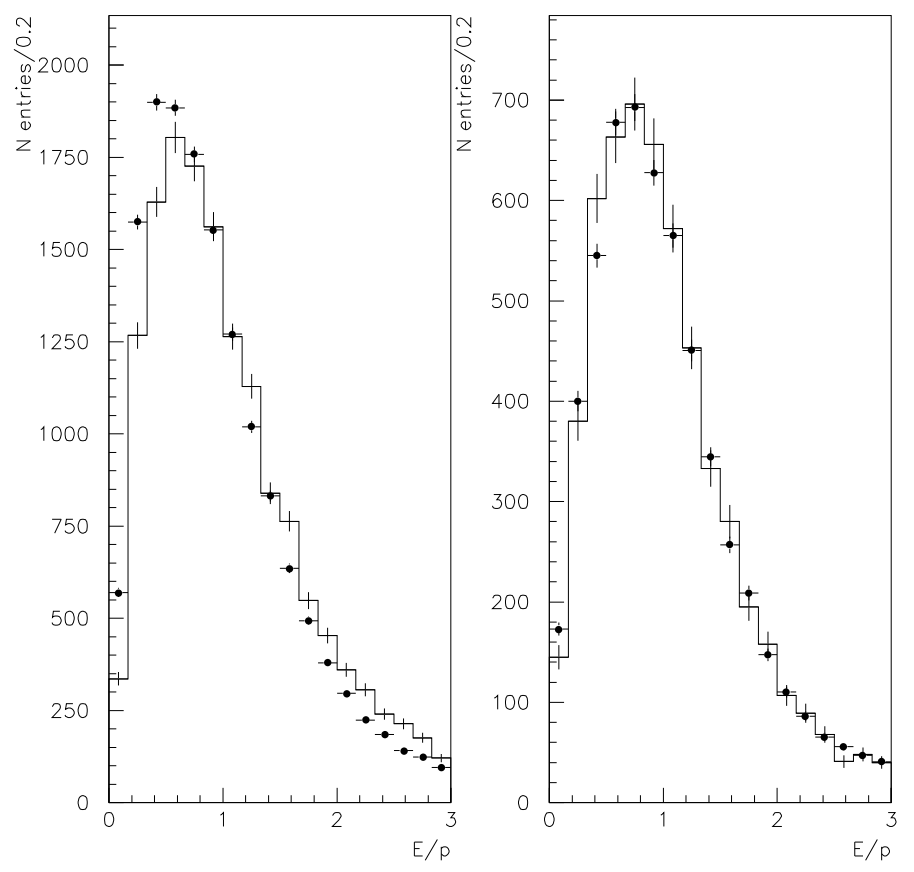

Figure 34: $E / p$ ratio in the HCAL (plus HPC) for charged particles with (left) $p<6 \mathrm{GeV} / c$ and (right) $p>6 \mathrm{GeV} / c$ in $\mathrm{Z}$ decays. Data (from the $1994 \mathrm{run}$ ) and simulation are shown by the points and histograms respectively. 
deposited in HPC plus HCAL to the momentum of the charged particle. Identified electrons and muons are excluded from the plot.

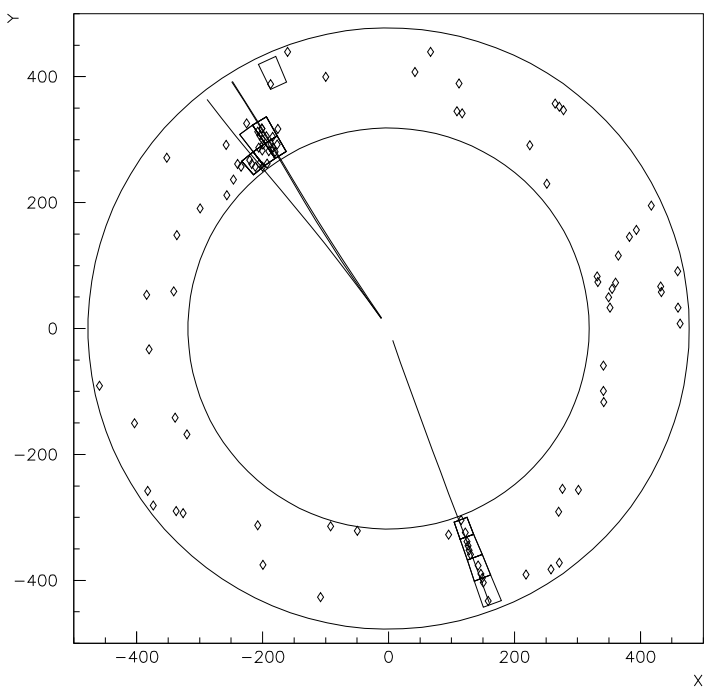

Figure 35: A $\tau^{+} \tau^{-}$event seen in the HCAL with cathode readout. One $\tau$ decays into three pions, the other one into a $\mu$.

Before 1994 only the pads were read out, as described above. To increase the granularity of the detector a new system has been developed which reads out the cathodes of individual streamer tubes. This new system is independent of the present pad readout and improves the granularity in $\phi$ by a factor of 3 and in $R$ by a factor of 5 . Combining the two readout systems will provide better $\pi / \mu$ separation, improved detection of neutral long lived particles, enhanced discrimination between neighbouring showers and more precise hadron energy measurement. This was demonstrated during a test on $4+4$ back-to-back barrel modules during the last 3 months of 1994 . The new system was available on the whole of the barrel at the start of 1995 [54]. The number of hits in the readout was been observed to be quasi-linearly correlated with the energy deposit up to $40 \mathrm{GeV}$. A typical event is presented in Figure 35. The endcaps will also be equipped with tube readout for the data taking in 1996.

\section{Energy Flow}

Non-leptonic decays of the $\mathrm{Z}$ give rise to relatively complex events containing charged and neutral hadrons, leptons from the weak decays of hadrons, and photons from electromagnetic decays. An understanding of the overall response of the DELPHI detector to these events is important in extracting the direction and energies of the partons in the event as well as a tag (or veto) for events that contain large amounts of missing energy. The energy flow in the event is determined by using all the information available from the tracking detectors and the calorimeters.

The precision of the energy flow measurement is a function of the intrinsic precision and resolution of the detectors, the efficiency of the detectors, and the efficiency of the 
reconstruction algorithms used to combine the data. Here we describe the method used to measure the energy flow in the detector.

In DELPHI the precision of the tracking detectors is such that (except for badly reconstructed tracks) the energy of charged particles is best estimated using the tracking detectors rather than the calorimeters. For these particles their masses are estimated using the standard DELPHI particle identification. The hadron calorimeters detect long lived neutral hadrons such as neutrons or $\mathrm{K}_{L}^{0}$ 's.

In general a charged particle will deposit energy in the calorimeters. An electron will deposit all of its energy in the electromagnetic calorimeter whereas a hadron may share its energy between the electromagnetic and hadronic calorimeters depending on the point in the detector that the hadronic shower is initiated. It is fundamental to differentiate hadronic showers from purely electromagnetic showers due to photons or electrons.

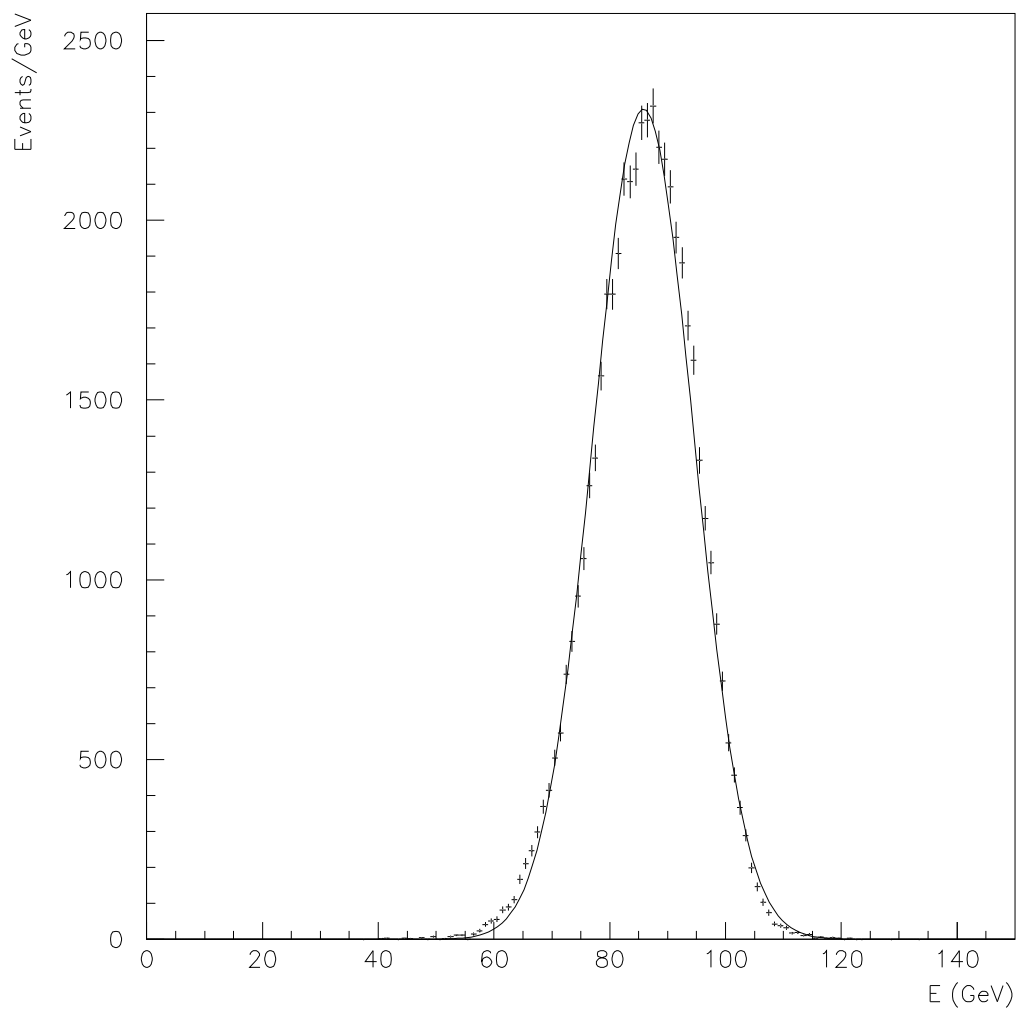

Figure 36: Distribution of the event energy after corrections.

The clusterization of energy is performed in two passes. First the electron and photon identification is performed (see Section 12). Showers in the HPC that are not identified as having come from a photon or electron are then reclustered according to the following algorithm. All the charged particle tracks in the event are sorted according to momentum. Each track, in order of descending energy, is then extrapolated through the electromagnetic calorimeters to the hadron calorimeter and any energy deposit within a specified angle $\left(2^{\circ}\right.$ for the HPC, $5^{\circ}$ for the FEMC and $6^{\circ}$ degrees for the HCAL, depending on the angular resolution of the detectors) of the extrapolated track is associated to that track. The process is repeated until all the charged particle tracks have been treated. 
The energy thus associated to the charged particle tracks is not used in evaluating the total energy - except for a special case discussed below.

In the next step, the remaining energy deposits are used to create neutral showers. These are "seeded" by ordering all remaining calorimeter hits by energy and using the highest energy hits first. The seed calorimeter hit is used in a similar fashion to the charged particle tracks: all calorimeter deposits within the specified angular separation (as above) of a straight line between the vertex and the seed hit are associated to the seed. When all the energy is exhausted the neutral showers are complete.

The neutral shower and charged particle information is then combined on an equal footing and passed through a jet-finding algorithm using LUCLUS, provided in the JETSET library[30]. The jet algorithm provides the four vector of each jet in the event. If any jet has more than the beam energy its momentum is scaled to make its energy equal to the beam energy. The total energy in the event is then calculated from the sum of the energies of all the jets in the event, and the missing momentum from the vector sum of their momenta.

At this stage the observed total energy of the event depends on the number of jets reconstructed in the event. The effect is well reproduced in simulated data. It arises partly because in narrow jets it is harder to distinguish neutrals in the calorimeters and the efficiency for reconstructing charged particles is also lower, and partly from having imposed a maximum jet energy. An overall correction is applied to the observed effect which depends on the number of jets. The added energy is about $8 \mathrm{GeV}$ for 2 jet events reducing to $0 \mathrm{GeV}$ for 6 jet events.

Additional corrections are made to account for the possibility of a charged particle being collinear with a neutral cluster. If the neutral particle has low energy there is no way to recover its energy as the discrepancy between the charged particle momentum $p$ (as measured in the tracker) and the associated calorimeter energy $E_{\text {ass }}$ is dominated by the large energy error in the calorimeter. However, if it has high energy the calorimetric energy associated to the particle will be much larger than its momentum. If the associated energy $E_{a s s}$ is more than $2.5 \sigma$ (in energy resolution) greater than the momentum $p$ a new neutral with energy $\left(E_{a s s}-p\right)$ is created and its energy is added to the event.

The total energy measurement precision for $Z$ events contained in the barrel is about $\pm 8.0 \mathrm{GeV}$ (see Figure 36) whereas in the forward region the uncertainty increases to about $\pm 10.5 \mathrm{GeV}$.

The directional precision (for jets) is computed using two jet events. The jets are collinear, in the transverse plane, to within 1.3 degrees. This corresponds to an individual jet having a directional resolution of about $0.9^{\circ}$.

In analyses where one can neglect the missing energy, for example in QCD analyses of 3-jet events or in studies of quasi-3-jet events at LEP2 where one "jet" is a radiated photon, one can compute the energies of the jets from their directions using momentum and energy conservation: most simply, assuming massless kinematics, the jet energies can be expressed as:

$$
p_{j}^{\text {calc }}=E_{j}^{\text {calc }}=\frac{\sin \theta_{j}}{\sin \theta_{1}+\sin \theta_{2}+\sin \theta_{3}} \sqrt{s}, \quad j=1,2,3
$$

where $\theta_{j}$ is the inter-jet angle opposite to the $j$-th jet [53]. Studies using simulation show that, for jet energies from $10 \mathrm{GeV}$ up to $40 \mathrm{GeV}$, the calculated jet energy $E_{j}^{\text {calc }}$ gives a better representation of the true jet energy than does the reconstructed (or visible) jet energy. The use of expression (8) both corrects for the underestimation of the jet energy due to particle losses and improves the energy resolution by about a factor 2 . 


\section{Muon Identification}

The iron of the hadron calorimeter provides a filter which gives a first level of separation between muons and hadrons. Most hadrons are stopped by this material, whereas all muons of momenta above $2 \mathrm{GeV} / \mathrm{c}$ are expected to penetrate to the Muon Chambers $(\mathrm{MUC}=\mathrm{MUB}+\mathrm{MUF}+\mathrm{SMC})$.

After this filtering there remains in the MUC a residual activity arising from hadronic tracks. Punch-through from hadronic showers, decays in flight and particles traversing the HCAL sector boundaries contribute to this background. For example, charged pions from three-prong $\tau$ decays give signals in the MUC on $\sim 5 \%$ of occasions. Because of the high ratio of charged hadrons to prompt leptons in $\mathrm{Z} \rightarrow q \bar{q}$ decays, further discrimination between hadrons and muons is necessary to achieve muon samples of acceptable purities in the jet environment. To this end, criteria can be placed on the goodness of association between the extrapolated candidate tracks and the signals in the MUC. Signals induced by hadronic showers and decays in flight of kaons will in general give larger deviations from the track extrapolation in position and direction than are expected from the multiple Coulomb scattering of a muon of the same momentum.

The DELPHI muon identification algorithm is based on these principles. Charged particle tracks, reconstructed in the central detectors, are extrapolated through the solenoid and the iron of the HCAL, referring to a map of the return field in this region. During this extrapolation the tracking errors are propagated; also the errors from multiple scattering are added. In both cases, correlations among the errors on the track parameters are taken into account. The extrapolations are made to software reference surfaces coincident with the MUC modules. From these, and from the knowledge of the MUC geometry, the extrapolated coordinates in $R \phi$ and $z$ are available at each muon chamber layer, together with the track direction in $\theta$ and $\phi$ (in the endcap these coordinates are given in $x$ and $y$.) In addition a full error matrix is provided at the innermost reference surface.

For each extrapolated track, the MUC hits in the event are searched through. A $\chi^{2}$ comparison is then made between a hit and the extrapolated track coordinates in that layer. Any hit potentially associated is flagged for further consideration. In this way a set of hits is selected for fitting.

A $\chi^{2}$ fit is then made at the innermost reference surface. The four track parameters are varied here and the effect of this variation is projected into the chambers themselves, where it is compared with the MUC hits in the two measured coordinates. The track covariance matrix and the assigned MUC measurement errors participate in this $\chi^{2}$. If the fit does not converge the worst hit is removed and the fit repeated. If there is more than one candidate hit in a layer, then the hit giving the fit with lowest $\chi^{2}$ is taken - it is this logic which resolves the left-right ambiguity in the chamber drift coordinate. The fit returns a set of associated hits, track and chamber residuals, and a $\chi^{2}$ per degree of freedom giving the goodness of association.

After these fits, the same MUC hit may be assigned to more than one track. These multiple associations, termed 'ambiguities', are clearly unphysical. Ambiguities are resolved by assigning the hit to the track with the greatest number of associated hits; if this still does not uniquely select a track then the $\chi^{2}$ values are compared.

Ideally this $\chi^{2}$ alone should possess sufficient discrimination to distinguish between prompt muons and hadrons. Inevitably, however, for genuine muons the set of associated hits can contain delta rays knocked from the chamber walls, wrongly included signals from overlapping hadronic showers or badly reconstructed space points from the occasional poorly calibrated chamber. These inflate the $\chi^{2}$ and degrade the muon-hadron separation. 
For this reason it is necessary to identify bad hits and remake the fit with these removed. What constitutes a "bad hit" depends on the performance required from the algorithm, as the discarding of hits reduces the efficiency of the tag. Four tag levels are defined, called very loose, loose, standard and tight. These are constructed to satisfy all muon identification requirements ranging from studies of isolated, low background channels like $\mathrm{Z} \rightarrow \mu^{+} \mu^{-}$(very loose) to those requiring high purity samples in hadronic jets (tight). Depending on the tag requested, poor hits with an individual $\chi^{2}$ above a certain cut are discarded, and the fit is remade. Finally, cuts are applied on the global $\chi^{2}$ from the fit and also on a $\chi^{2}$ which represents the quality of the match between the fitted track and the initial extrapolation. The severity of these cuts again depends on the tag level. The standard and tight tags also require an associated hit in at least one of the MUC modules lying outside the iron.

Inefficiencies are introduced into the simulation to model correctly the performance of the detectors in the data and the loss of hits sustained in the refit. Smearings and error assignments are made so that the data distributions are well reproduced in the key variables (e.g., number of associated hits in the MUC, and global and extrapolation $\chi^{2}$ of the fit).

The muon identification efficiency is studied in data and simulation using the following channels:

- $\mathrm{Z} \rightarrow \mu^{+} \mu^{-}$events, giving muons with momentum $\sim 45 \mathrm{GeV} / c$;

- $\mathrm{Z} \rightarrow \tau^{+} \tau^{-}$events where at least one of the $\tau \rightarrow \mu \nu_{\tau} \bar{\nu}_{\mu}$, giving muons with a continuous momentum spectrum between $3 \mathrm{GeV} / c$ and $\sim 40 \mathrm{GeV} / c$;

- $\gamma \gamma \rightarrow \mu^{+} \mu^{-}$events, giving low momentum muons produced mainly in the forward region.

The kinematics and topologies of these decays allow samples to be isolated with high purity. In these selections the muon candidates are tagged using their energy deposition in the electromagnetic and hadron calorimeters, thereby yielding unbiased samples for determining the efficiency of the algorithm described above.

The performance is given only for muons with momentum above $3 \mathrm{GeV} / c$ and polar angle $\theta$ inside the angular acceptance of the $\operatorname{MUB}\left(53.0^{\circ} \leq \theta \leq 88.5^{\circ}\right.$ and $91.5^{\circ} \leq \theta \leq$ $\left.127.0^{\circ}\right)$ or the MUF $\left(20.0^{\circ} \leq \theta \leq 42.0^{\circ}\right.$ and $\left.138.0^{\circ} \leq \theta \leq 160.0^{\circ}\right)$. Figure 37 shows the efficiency of the standard tag in the data and in the simulation.

The probability of misidentifying a charged hadron as a muon is measured using:

- $\mathrm{Z} \rightarrow \tau^{+} \tau^{-}$events where one $\tau$ decays to three charged pions, $\tau \rightarrow 3 \pi \nu_{\tau}$, and the other to only one charged particle;

- hadronic Z decays containing a $\mathrm{K}^{0} \rightarrow \pi^{+} \pi^{-}$decay.

These are selected with high purity and any residual muon contamination is corrected for using the simulation.

Table 9 shows the efficiencies and misidentification probabilities for the four tags, averaged over the MUB and MUF. It is seen that a spectrum of well determined tagging performances is available. More details can be found, for example, in [55].

The recent addition of the SMC has improved the hermeticity of the DELPHI muon identification. This detector gives coverage in the region between the barrel and the endcap. Figure 37(c) shows the efficiency of the very loose tag in this region before and after the installation of the SMC. 

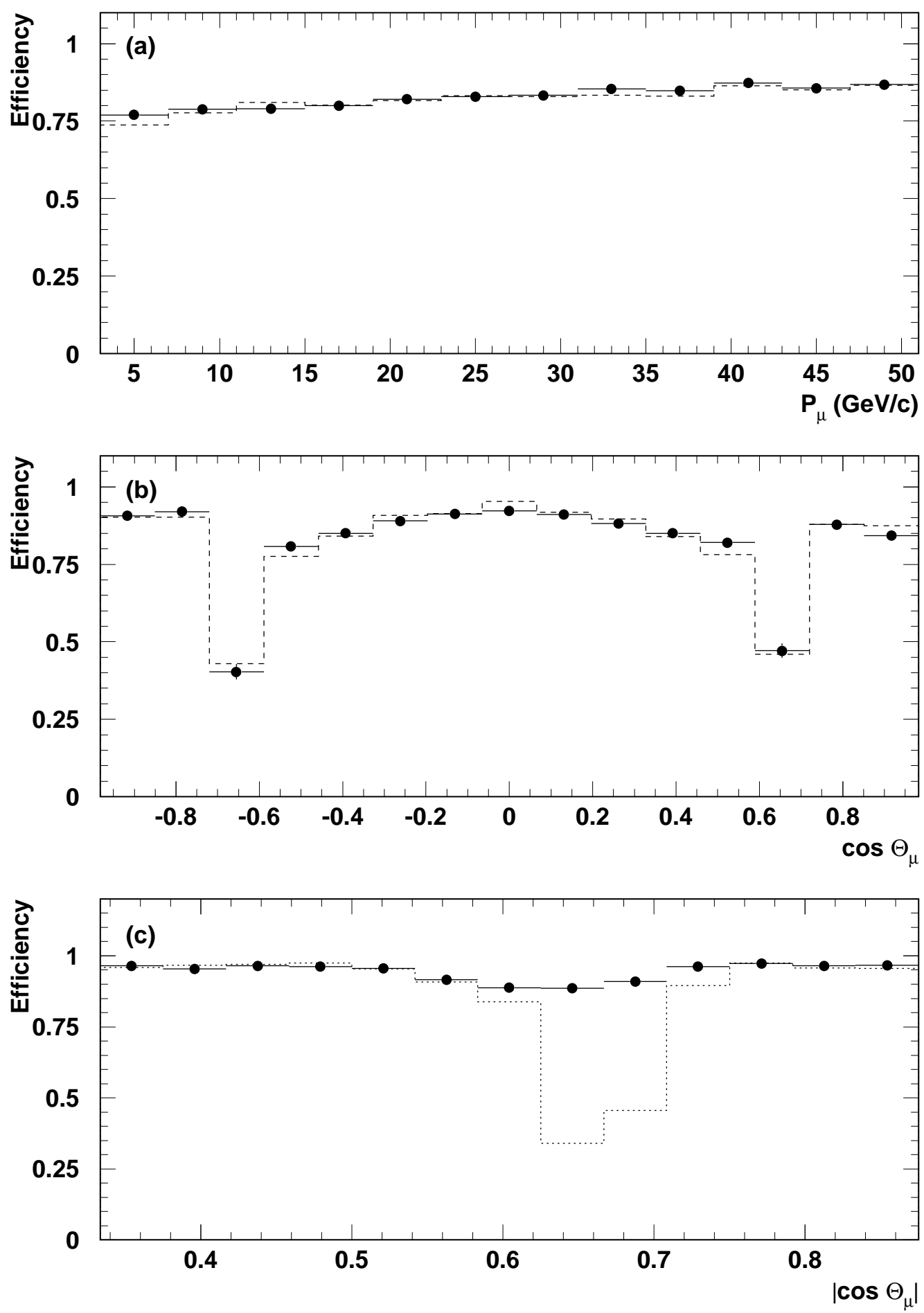

Figure 37: Muon identification efficiency with the standard tag as a function of muon momentum (a) and of polar angle (b). The results obtained for real data are represented by the points, the dashed line shows the results obtained for simulated data. In (c) is shown the performance of the very loose tag in the region of the SMC. The points show the efficiency with the SMC present, and the broken line shows the efficiency with the MUB and MUF alone. 


\begin{tabular}{|l|c|c|}
\hline Tag & Efficiency (\%) & Misid. Probability (\%) \\
\hline Very loose & $95.9 \pm 0.1$ & $5.4 \pm 0.2$ \\
Loose & $94.8 \pm 0.1$ & $1.5 \pm 0.1$ \\
Standard & $86.1 \pm 0.2$ & $0.7 \pm 0.1$ \\
Tight & $76.0 \pm 0.2$ & $0.4 \pm 0.1$ \\
\hline
\end{tabular}

Table 9: Identification efficiency for each tag as determined on $\mathrm{Z} \rightarrow \mu^{+} \mu^{-}$and misidentification probabilities as determined on $\mathrm{Z} \rightarrow \tau^{+} \tau^{-}$events with $\tau \rightarrow 3 \pi \nu_{\tau}$.

\section{Event Selection for Physics Analysis}

The event selections vary in the different physics analyses but most derive from the prototypes described below. Additional cuts are often imposed, limiting the angular acceptance to that of particular subdetectors most essential to the analysis and requiring them to be fully operational.

\subsection{Hadronic Event Selection}

For selecting hadronic events, basically two sets of selections are used.

The first one (the "Team 10" selection) is meant to guarantee a reliable calculation of event shape variables and is the basis of the selections used in QCD papers. For the event selection, charged particles are accepted in the momentum range $0.2<p<50 \mathrm{GeV} / c$ provided the relative momentum error is below 1 and the length seen in the tracking detectors exceeds $50 \mathrm{~cm}$. The polar angle $\theta$ of the charged particle must be between $25^{\circ}$ and $155^{\circ}$ and its impact parameter with respect to the average interaction point must be below $5 \mathrm{~cm}$ in the plane transverse to the beam and $10 \mathrm{~cm}$ along the beam. A hadronic event is selected by requiring a charged particle multiplicity above 4 and a total energy in charged particles (assumed to be pions) above $15 \mathrm{GeV}$ with at least $3 \mathrm{GeV}$ in each of the hemispheres $\theta<90^{\circ}$ and $\theta>90^{\circ}$. The efficiency for selecting hadronic $Z$ decays is over $90 \%$ and the background, mainly from $\tau^{+} \tau^{-}$and $\gamma \gamma$ events and evaluated by simulation of these processes, is below $0.3 \%$.

The second one (the "Team 4" selection) is meant to guarantee a high and well understood efficiency and is the basis of the selections used for measurements of the $\mathrm{e}^{+} \mathrm{e}^{-} \rightarrow Z^{0} \rightarrow$ hadrons cross section. Hadronic $Z$ decays are selected as events with a multiplicity above 4 of charged particles with $p>400 \mathrm{MeV} / c, 20^{\circ}<\theta<160^{\circ}$ and a track length of at least $30 \mathrm{~cm}$ in the TPC, with a total energy in these charged particles above $0.12 \times E_{c m}$, and with a thrust axis well clear of the beampipe, ie satisfying $\left|\cos \theta_{\text {thrust }}\right|<0.95$. The efficiency for selecting hadronic $\mathrm{Z}$ decays is over $95 \%$. The background, again mainly from $\tau^{+} \tau^{-}$pairs but also from $\gamma \gamma$ collisions, is below $0.7 \%$.

\subsection{Leptonic Event Selection}

The selections of leptonic events described below follow those used in [24] for the determination of the $\mathrm{e}^{+} \mathrm{e}^{-} \rightarrow Z^{0} \rightarrow \mathrm{e}^{+} \mathrm{e}^{-}, \mathrm{e}^{+} \mathrm{e}^{-} \rightarrow Z^{0} \rightarrow \mu^{+} \mu^{-}$, and $\mathrm{e}^{+} \mathrm{e}^{-} \rightarrow Z^{0} \rightarrow \tau^{+} \tau^{-}$ cross sections. 


\subsection{1 $e^{+} e^{-}$Event Selection}

Two independent methods have been developed to select $\mathrm{e}^{+} \mathrm{e}^{-} \rightarrow \mathrm{e}^{+} \mathrm{e}^{-}$events, so as to increase the overall selection efficiency and to obtain a better determination of the efficiency corrections. The first one is described below.

Events with two charged particles were considered if both particles had a polar angle between $44^{\circ}$ and $136^{\circ}$ and they were back-to-back, ie if their acollinearity was smaller than $10^{\circ}$. Events were accepted if they contained

- two back-to-back high energy clusters in the HPC, at least one with energy above $30 \mathrm{GeV}$, the other above $25 \mathrm{GeV}$;

- no more than 4 charged particles with momentum above $1.5 \mathrm{GeV}$ and impact parameter below $5 \mathrm{~cm}$ both in the radial and in the beam direction;

- total electromagnetic energy above $70 \mathrm{GeV}$ in $1-3,0-3$ or $0-4$ topologies (the numbers are the number of charged tracks in each hemisphere);

- hits in the VD compatible with one charged particle per hemisphere in 0-0 and 0-1 topologies.

To avoid losing events due to bad reconstruction of one shower in the HPC, events were also accepted with

- one very energetic electromagnetic cluster with energy above $40 \mathrm{GeV}$;

- at least one charged particle in each hemisphere;

- no energy deposited beyond the first 1.5 interaction lengths of the HCAL;

The selection efficiency was about $90 \%$ and the percentage of background (mostly from $\tau^{+} \tau^{-}$events) was about $1.6 \%$.

\subsection{2 $\mu^{+} \mu^{-}$Event Selection}

Events with two charged particles in the angular range $11^{\circ}<0<169^{\circ}$, or $20^{\circ}<\theta<$ $160^{\circ}$, were kept if

- both particles had momenta above $15 \mathrm{GeV}$ and came from the interaction region (the size taken for this region depended on which detectors participated in the track fit);

- the acollinearity angle between the two charged particle tracks was below $10^{\circ}$;

- there were no additional charged particles with momenta above $5 \mathrm{GeV}$, unless the fastest particle had a momentum over $40 \mathrm{GeV} / c$ (to reduce loss of muon pairs in which the third particle was due to radiative $e^{+} e^{-}$pair creation).

Each particle had to be identified as a muon by either the MUC, HCAL, HPC or FEMC. If either particle was identified as a hadron by the HCAL or if both particles deposited more than $10 \mathrm{GeV}$ in the HPC or FEMC, and their acollinearity exceeded $1^{\circ}$, the event was rejected. The cosmic ray background was substantially reduced by requiring both tracks to be consistent with having been produced at the beam crossover time, using the timing measurements from the TPC and the OD, and by cuts on the distance of closest approach to the average interaction point in the transverse plane. made using the microvertex detector,

The overall muon selection efficiency was about $94 \%$ and the background (again mostly from $\tau^{+} \tau^{-}$events) was below $3 \%$. 


\subsection{3 $\tau^{+} \tau^{-}$Event Selection}

To select $\mathrm{Z} \rightarrow \tau^{+} \tau^{-}$events, each event was divided into two hemispheres relative to its thrust axis and the most energetic charged particle (leading track) in each hemisphere was chosen to define the corresponding $\tau$ quantities. The leading track in at least one of the hemispheres had to be in the accepted polar angle range, $43^{\circ}<\theta<137^{\circ}$. To define calorimetric energies, all energy inside a cone of $30^{\circ}$ half angle around the leading track was added as the $\tau$ energy.

A first set of cuts was applied to remove hadronic $\mathrm{Z}$ decays and two-photon events:

$$
2 \leq N_{c h} \leq 6, \theta_{\text {iso }} \geq 160^{\circ}, E_{\text {vis }}>8 \mathrm{GeV},
$$

where $N_{c h}$ is the number of charged particles reconstructed in the TPC and coming from near the average interaction point $(r<5 \mathrm{~cm}$ and $|z|<10 \mathrm{~cm}), \theta_{\text {iso }}$ is the smallest angle between two charged particles in opposite hemispheres, and $E_{\text {vis }}$ is the total energy, defined as the sum of the charged particle momenta and neutral electromagnetic energy.

Two further cuts were used to reject leptonic Z decays, $e^{+} e^{-}(\gamma)$ and $\mu^{+} \mu^{-}(\gamma)$ :

$$
P_{\text {rad }}<1.0, E_{\text {rad }}<1.0
$$

where $P_{\text {rad }}=\frac{\sqrt{p_{1}^{2}+p_{2}^{2}}}{P_{\text {beam }}}$ and $E_{\text {rad }}=\frac{\sqrt{E_{1}^{2}+E_{2}^{2}}}{E_{\text {beam }}}, p_{1}\left(p_{2}\right)$ and $E_{1}\left(E_{2}\right)$ being the momentum and electromagnetic energy assigned to each $\tau$ as explained above.

Cosmic, beam-gas and beam-wall events were rejected with impact parameter cuts:

$$
r_{1}<1.5 \mathrm{~cm}, r_{2}<1.5 \mathrm{~cm},\left|z_{1}\right|<4.5 \mathrm{~cm},\left|z_{2}\right|<4.5 \mathrm{~cm}
$$

where $r_{1}$ and $r_{2}$ are the impact parameters in the transverse plane of the two leading tracks with respect to the average interaction point and $z_{1}$ and $z_{2}$ are the distances along the beam between the point of closest approach and the interaction point.

Finally, extra cuts were applied to 1-1 topology events, to remove remaining background

$$
\theta_{\text {acol }}>0.5^{\circ},\left|\vec{P}_{T}\right|>0.4 \mathrm{GeV},\left|z_{1}-z_{2}\right|<3 \mathrm{~cm},
$$

where $\left|\vec{P}_{T}\right|$ is the resultant momentum transverse to the beam axis, and $\theta_{\text {acol }}$ is the acollinearity angle of the two charged particles. The acollinearity cut reduces the dilepton and cosmic ray backgrounds, the $\left|z_{1}-z_{2}\right|$ cut further reduces the cosmic ray background, and the $\left|\vec{P}_{T}\right|$ cut reduces the two-photon background.

The selection efficiency was about $53 \%$, equivalent to about $82 \%$ for polar angles $43^{\circ}<\theta<137^{\circ}$. The overall background was $(1.9 \pm 0.4) \%$ from other $\mathrm{Z}$ decays, $2.0 \pm 0.6$ $\mathrm{pb}$ from two-photon events, and up to $2.1 \mathrm{pb}$, depending on centre-of-mass energy, from Bhabha processes.

\section{Conclusions}

The DELPHI detector has operated with high efficiency throughout the six years of LEP1 operation. It has demonstrated a performance that is well up to design specifications and has allowed and is allowing the extraction of physics results in all sectors, in particular, since the successful completion and operation of the RICH system, the ones based on identified final states from $Z$ decays. This performance, currently being further augmented by a program of upgrades planned in 1992 and now reaching completion and by continual development of the software, make it a powerful instrument for future physics studies at LEP2. 


\section{Acknowledgements}

We are greatly indebted to our technical collaborators and to the funding agencies for their support in building and operating the DELPHI detector, and to the members of the CERN-SL Division for the excellent performance of the LEP collider. 
[1] P. Aarnio et al. (DELPHI Collaboration), Nucl. Instr. and Meth. A303 (1991) 233.

[2] N. Bingefors et al., Nucl. Instr. and Meth. A328 (1993) 447.

[3] V. Chabaud et al., Nucl. Instr. and Meth. A368 (1996) 314.

[4] DELPHI Collaboration, "An Upgrade of DELPHI in the Forward Region - Letter of Intent", CERN/LEPC/92-5, LEPC/I-II, 1992.

[5] The DELPHI Collaboration, "Proposal for the Replacement of the Small Angle Calorimeter of DELPHI", CERN/LEPC/92-6, 1992;

S.J. Alvsvaag et al., "The DELPHI Small Angle Tile Calorimeter", contribution to the IEEE Nuclear Science Symposium and Medical Imaging Conference, Norfolk, USA, 1994, Submitted to IEEE Trans. Nucl. Sci. (DELPHI 94-157/CAL 120);

The DELPHI Collaboration, "Performance of the new high precision luminosity monitor of DELPHI at LEP", contributed paper eps0528 to the EPS-HEP Conference, Brussels 1995 (DELPHI 95-68/PHYS 503).

[6] The DELPHI Collaboration, Proposal for the DELPHI Surround Muon Chambers, DELPHI 92-139/TRACK 71, October 1992.

[7] V. Bocci et al., Nucl. Instr. and Meth. A362 (1995) 361;

V. Bocci et al., Basic Concepts and Architectural Details of the DELPHI Trigger System, CERN/ECP 94-18, contribution to the Nuclear Science Symposium and Medical Imaging Conference, Norfolk, USA, 1994. Submitted to IEEE Trans. Nucl. Sci.

[8] Ph. Charpentier et al., "The DELPHI Fastbus Data Acquisition System", Proc. Int. Conf. on Computing in High Energy Physics 1991 (CHEP91, Tsukuba, Japan), Y. Watase and F. Abe eds., Universal Academy Press, Tokio 1991, p. 643; (DELPHI 91-92/DAS 112);

M. Jonker et al., "Architecture and Performance of the DELPHI Data Acquisition and Control System", Proc. Int. Conf. on Computing in High Energy Physics 1991 (CHEP91, Tsukuba, Japan), Y. Watase and F. Abe eds., Universal Academy Press, Tokio 1991, p. 619 (DELPHI 91-93/DAS 113);

M. Jonker et al., "DELPHI Experiment Control System", Proc. Artificial Intelligence and Expert Systems in High Energy and Nuclear Physics (L'Agelonde, France), D. Perret-Gallix ed., World Scientific 1992, p. 543; (DELPHI 92-98/DAS $127)$;

C. Gaspar, "DELPHI Experiment Control Integration", Proc. Artificial Intelligence and Expert Systems in High Energy and Nuclear Physics (L'Agelonde, France), D. Perret-Gallix ed., World Scientific 1992, p. 553; (DELPHI 92-99/DAS 128).

[9] T.J. Adye et al., Nucl. Instr. and Meth. A349 (1994) 160; expanded in "The Design and Operation of the Slow Controls for the DELPHI Experiment at LEP", DELPHI 94-14/DAS 151, 1994.

[10] G. Delavallade and J.P. Vanuxem, "The LTD: a Fastbus time digitizer for LEP detectors", CERN-EP/86-23.

[11] R. Brun and J. Zoll, "ZEBRA User Guide", CERN Program Library 1994.

[12] "DELANA User's Guide", DELPHI 89-44/PROG 137, 1989.

[13] J. Barlow et al., IEEE Trans. Nucl. Sci. 36 (1989) 1549.

[14] M. Dönszelmann, C. Gaspar and J. Valls, "A Configurable Motif Interface for the DELPHI Experiment at LEP", MOTIF '92, Second Int. Conf. On Motif Application Development and Use (Washington D.C., USA, 1992); DELPHI 92-150/DAS 134.

[15] B. Franek et al., CHEP94 Proceedings (LBL-35822 CONF-940922 UC-405) p. 130.

[16] C. Gaspar and M. Dönszelmann, "DIM - A distributed Information Management 
System for the DELPHI Experiment at CERN" Proc. IEEE Conf. REAL TIME '93 on Computer Applications in Nuclear, Particle and Plasma Physics, (Vancouver, Canada), D. Axen and R. Poutissou eds., p. 156; (DELPHI 94-4/DAS 148);

M. Dönszelmann and C. Gaspar, Nucl. Instr. and Meth. A352 (1994) 280.

[17] G. Smith, "DELPHI Slow Controls G64 Microcomputers Skeleton Program", DELPHI 94-13/DAS-150, rev. 1994.

[18] Costruzioni Apparecchiature Elettroniche Nucleari (CAEN) S.p.A., Via Vetraia, 11 - 55049 VIAREGGIO (Italy).

[19] P. Burkimsher, "EMU, The MODEL Error Message Utility", CERN/ECP Writeup, December 1990.

[20] T. Adye et al., Comp. Phys. Comm. 57 (1989) 466.

[21] T.J. Berners-Lee, IEEE Trans. Nucl. Sci. 34 (1987) 1050.

[22] Yu. Belokopytov and V. Perevozchikov, "CARGO Database Management Package", DELPHI 93-5/PROG 195, 1993;

Yu. Belokopytov et al., "The DELPHI Database Description: Calibration \& Detector Status File", DELPHI 93-4/PROG-194, 1993.

[23] R. Brun et al., "PAW - Physics Analysis Workstation: The Complete Reference", CERN Computer centre Program Library Writeup Entry Q121, upd. 1994.

[24] P. Abreu et al. (DELPHI Collaboration), Nucl. Phys. B417 (1994) 3, erratum ibid., B426 (1994) 244.

[25] P. Abreu et al. (DELPHI Collaboration), Nucl. Phys. B418 (1994) 403;

D. Bardin et al. (DELPHI Collaboration), "Precision Determination of the Z Resonance Parameters", DELPHI 94-114 PHYS 431, Contributed paper gls0301 to the 27th International Conference on High Energy Physics, Glasgow (UK) 1994.

[26] DELPHI Collaboration, "High precision relative luminosity measurement with a Very Small Angle Tagger (VSAT) in DELPHI", DELPHI 92-77 PHYS 188, Contributed paper to the Dallas Conference, August 1992.

[27] S. Jadach et al., "Monte Carlo program BHLUMI 2.01 for Bhabha scattering at low angles with Yennie-Frautschi-Suura exponentiation", CERN-TH 6230/91 (1991).

[28] F. A. Berends, W. Hollik and R.Kleiss, Nucl. Phys. B304 (1988) 712.

[29] "DELSIM User Manual", DELPHI 87-96/PROG 99, 1989; "DELSIM Reference Manual", DELPHI 87-98/PROG 100, 1989.

[30] T. Sjöstrand, Comp. Phys. Comm. 82 (1994) 74.

[31] G. Marchesini and B.R. Webber, Nucl. Phys. B238 (1984) 1;

G. Marchesini et al., Comp. Phys. Comm. 67 (1992) 465.

[32] L. Lonnblad, Comp. Phys. Comm. 71 (1992) 15.

[33] J.E. Campagne and R. Zitoun, Z. Phys. C43 (1989) 469, and Proc. of the Brighton Workshop on Radiative Corrections, Sussex, July 1989.

[34] S. Jadach et al., Comp. Phys. Comm. 79 (1994) 503.

[35] K. Hamacher and M. Weierstall, "Tuning and Test of Fragmentation Models Based on Identified Particles and Precision Event Shape Data", DELPHI 95-80/PHYS 515, contributed paper eps0548 to the EPS-HEP Conference, Brussels 1995.

[36] Yu. Belokopytov, S. Gumenyuk and V. Perevozchikov, "DELPHI Detector Description Application Package User Manual" DELPHI 90-37/PROG 154, 1990.

[37] F. Carena and G. Gopal, "CARGO User Manual", DELPHI 86-28/PROG 46, 1986.

[38] R. Brun et al., CERN Report DD/EE/84-1 (1986).

[39] W.R. Nelson et al., "EGS4 User Manual", SLAC-265 (1985).

[40] J. Cuevas et al., Nucl. Instr. and Meth. A274 (1989) 459.

[41] D. Bertrand and L. Pape, "TANAGRA User's Guide", DELPHI 87-95/PROG 98, 
1989.

[42] M. Crozon and J. Maillard, "A Fast Algorithm to Reconstruct Tracks in the TPC", DELPHI 83-58 PROG, 1983.

[43] P. Billoir, Nucl. Instr. and Meth. 225 (1984) 352.

[44] P. Abreu et al. (DELPHI Collaboration), Z. Phys. C65 (1995) 555;

G.V. Borisov, "Lifetime Tag of Events $Z \rightarrow b \bar{b}$ with the DELPHI Detector", DELPHI 94-125/PROG 208, 1994;

G.V. Borisov and C. Mariotti, "Fine tuning of the impact parameter resolution in the DELPHI detector", DELPHI 95-140/PHYS 565, 1995.

[45] L. Chevalier, "Particles identification using their ionization in the time projection chamber of DELPHI", PhD thesis, DAPNIA/SPP 92-32, Saclay (1992).

[46] W. Adam et al., Nucl. Instr. and Meth. A343 (1994) 68;

E.G. Anassontzis et al., Nucl. Instr. and Meth. A323 (1992) 351;

W. Adam et al., Nucl. Instr. and Meth. A338 (1994) 284;

DELPHI RICH Collaboration, contributions to the Second Workshop on RICH Detectors, Uppsala 1995.

[47] P. Baillon, Nucl. Instr. and Meth. A238 (1985) 341;

P. Abreu et al. (DELPHI Collaboration), Z. Phys. C67 (1995) 1.

[48] P. Abreu et al. (DELPHI Collaboration), Phys. Lett. B334 (1994) 435.

[49] M. Berggren et al., Nucl. Instr. and Meth. 225 (1984) 477;

A. Cattai et al., Nucl. Instr. and Meth. A235 (1985) 310;

V. Gracco et. al., Nucl. Instr. and Meth. A252 (1986) 572;

F.L. Navarria et al., Nucl. Instr. and Meth. A257 (1987) 499;

H.G. Fischer, Nucl. Instr. and Meth. A265 (1988) 218;

A. Algeri et al., "Performance of the HPC Calorimeter in DELPHI", CERNPPE/95-04 (1995).

[50] K. Cankocak et al., "Study of the $40^{\circ}$ counters", DELPHI 95-152/CAL 127, 1995.

[51] A. De Angelis, "Light Quark Hadrons in Hadronic Z Decays", CERN-PPE/95-135, 1995, to be published in the Proceedings of the EPS-HEP Conference, Brussels 1995.

[52] P. Abreu et al. (DELPHI Collaboration), "Measurement of Inclusive $\pi^{0}$ Production in Hadronic Z Decays", CERN-PPE/95-144, submitted to Z. Phys. C.

[53] P. Abreu et al. (DELPHI Collaboration), "Energy Dependence of the Differences between Quark and Gluon Jet Fragmentation", CERN-PPE/95-164, submitted to Z. Phys. C.

[54] I. Ajinenko et al., "The cathode read-out of the DELPHI hadron calorimeter", DELPHI 94-45/CAL 114, 1994.

[55] G.R. Wilkinson and P. Collins, "MUCFIX - Refining the Muon Chamber Information on the DST", DELPHI 93-13/PHYS 262, 1993;

H. De Boeck and G.R. Wilkinson, "MUFLAG: A framework for Muon Identification", DELPHI 93-14/PHYS 263, 1993;

F. Stichelbaut and G.R. Wilkinson, "Performance of muon identification in DELPHI for the 93 and 94 data", DELPHI 95-140/PHYS 565, 1995. 